\title{
DIOPHANTINE GEOMETRY OVER GROUPS I: MAKANIN-RAZBOROV DIAGRAMS
}

\author{
by ZLIL SELA $^{(1)}$
}

\begin{abstract}
This paper is the first in a sequence on the structure of sets of solutions to systems of equations in a free group, projections of such sets, and the structure of elementary sets defined over a free group. In the first paper we present the (canonical) Makanin-Razborov diagram that encodes the set of solutions of a system of equations. We continue by studying parametric families of sets of solutions, and associate with such a family a canonical graded Makanin-Razborov diagram, that encodes the collection of Makanin-Razborov diagrams associated with the individual members in the parametric family.
\end{abstract}

Sets of solutions to equations defined over a free group have been studied extensively, mostly since Alfred Tarski presented his fundamental questions on the elementary theory of free groups around 1945. Considerable progress in the study of such sets of solutions was made by G. S. Makanin, who constructed an algorithm that decides if a system of equations defined over a free group has a solution [Mal], and showed that the universal and positive theories of a free group are decidable [Ma2]. A. A. Razborov was able to give a description of the entire set of solutions to a system of equations defined over a free group [Ral], a description that was further developed by $\mathrm{O}$. Kharlampovich and A. Myasnikov [Kh-My].

A set of solutions to equations defined over a free group is clearly a discrete set, and all the previous techniques and methods that studied these sets are combinatorial in nature. Naturally, the structure of sets of solutions defined over a free group is very different than the structure of sets of solutions (varieties) to systems of equations defined over the complexes, reals or a number field. Still, perhaps surprisingly, concepts from complex algebraic geometry and from Diophantine geometry can be borrowed to study varieties defined over a free group.

In this paper we start a sequence that borrows concepts and techniques from geometric group theory, low dimensional topology, and Diophantine geometry to study the structure of varieties defined over a free group. Our techniques and point of view on the study of these varieties is rather different than any of the pre-existing techniques in this field, though, as one can expect, some of our preliminary results overlap with previously known ones. As we will see in this paper and mostly in the following ones, the techniques and concepts we use enable the study of the structure of varieties defined over a free group and their projections, and in particular, give us the possibility to answer some questions that seem to be essential in any attempt to understand the structure of elementary sentences and predicates defined over a free

(1) Partially supported by an Israel academy of sciences fellowship. 
group. At this stage, at least as it seems to us, none of the pre-existing (combinatorial) techniques can approach the difficulties involved in tackling these questions.

The sequence of papers on Diophantine geometry over groups is organized as follows. In the first paper we associate a canonical Makanin-Razborov diagram with a given system of equations defined over a free group. This Makanin-Razborov diagram encodes the entire set of solutions to the system. Later on we study systems of equations with parameters. With each such system we associate a (canonical) graded MakaninRazborov diagram, that encodes the Makanin-Razborov diagrams of the systems of equations associated with each specialization of the defining parameters. In the second paper of the sequence, we generalize Merzlyakov theorem on the existence of a formal solution associated with a positive sentence [Me]. We first construct a formal solution to a general AE sentence which is known to be true over some variety, and then develop tools that enable us to analyze the collection of all such formal solutions. In the third paper, we use techniques developed in the first two, to further study exceptional solutions of parametric systems of equations, which are one of the inherent pathologies in the theory that must be understood in studying the first order theory of a free group. Perhaps the main result of this paper is the existence of a global bound (independent of the parameters specialization) on the number of families of exceptional solutions. In the fourth paper we study $\mathrm{AE}$ sentences, and present a terminating iterative procedure for validation of such sentences. This terminating iterative procedure is the basis for our analysis of elementary sets defined over a free group presented in the last two papers. In the fifth paper we analyze the Boolean Algebra of AE sets and show that this Boolean algebra is invariant under projections. This implies that every elementary set defined over a free group is in the Boolean algebra of $\mathrm{AE}$ sets. The sixth paper uses the results of the fifth one to study elementary sets defined over a free group. In particular, we answer affirmatively some of A. Tarski's problems on the elementary theory of a free group, and obtain a classification of the f.g. groups that are elementary equivalent to a non-abelian f.g. free group. Throughout this paper, we use the abbreviation f.g. for finitely generated and f.p. for finitely presented.

We start the first paper of the sequence by studying limit groups that are obtained from (Gromov) limits of sequences of homomorphisms. In section 2 we study the (canonical) abelian JSJ decomposition of these groups, which is shown to be nontrivial. In the third section we study the (canonical) cyclic JSJ decomposition of a limit group, and show that this cyclic decomposition can not be trivial as well. In the fourth section we use the canonical cyclic JSJ decomposition to associate an analysis lattice with a limit group, which, in particular, implies that limit groups are finitely presented, and that a f.g. group is a limit group if and only if it is an $\omega$-residually free group (f.g. $\omega$-residually free groups were also shown to be f.p. by Kharlampovich and Myasnikov in [Kh-My]). The fifth section associates a canonical Makanin-Razborov diagram with a limit group. The properties of this diagram are similar to the ones 
obtained by Razborov [Ra2], and Kharlampovich and Myasnikov [Kh-My]. However, for the structure theory we develop, and for analyzing elementary predicates, it seems essential to have its canonical properties (which can not be guaranteed by combinatorial approaches), and to be able to have a "close relation" between the algebraic structure of a limit group and some of its (canonical) quotients with the structure of the diagram and the modular groups associated with it. In the sixth section we present a family of $\omega$-residually free groups that is more geometric in nature. The family of towers we present, plays an essential role when we classify f.g. groups that are elementary equivalent to a non-abelian free group in the last paper in the sequence. In section 7 we use our study of limit groups to study residually free groups.

At this point we begin our study of systems of equations with parameters. In section 10 we present graded limit groups, and in section 11 we associate a canonical graded Makanin-Razborov diagram with a graded limit group. We view the set of solutions to a system of equations with parameters as a bundle, where the groups of parameters lies in the base of this bundle. The graded Makanin-Razborov diagram is then a (flat) "connection" that allows one to compare different fibers associated with different specializations of the defining parameters. Section 11 studies the singular locus of that bundle, which is equivalent to studying the degeneracies in the graded Makanin-Razborov diagram. Section 12 generalizes our study of graded limit groups and diagrams to the study of multi-graded ones. Graded and multi-graded limit groups and diagrams are the basic objects needed in our analysis of sentences and elementary sets defined over a free group.

Quite a few people helped us while this work was conducted. We would like to thank Mladen Bestvina, Mark Feighn, Etienne Ghys, and the referees for their comments, corrections and suggestions that improved the presentation considerably. Especially, I am indebted to my former advisor Eliyahu Rips who introduced me to Tarski's problems, shared his knowledge and ideas with me, and suggested the possibility of a connection between the JSJ theory and the study of varieties defined over a free group, not long after the JSJ decomposition was introduced.

\section{Limit Groups}

Following [Ral] with a finite system of equations $\Phi$ over a free group $\mathrm{F}_{k}=$ $<a_{1}, \ldots, a_{k}>$ it is natural to associate a f.p. group $G(\Phi)$. If the system $\Phi$ is defined by the coefficients $a_{1}, \ldots, a_{k}$, the unknowns $x_{1}, \ldots, x_{n}$ and the equations:

$$
\begin{gathered}
w_{1}\left(a_{1}, \ldots, a_{k}, x_{1}, \ldots, x_{n}\right)=1 \\
\vdots \\
w_{s}\left(a_{1}, \ldots, a_{k}, x_{1}, \ldots, x_{n}\right)=1
\end{gathered}
$$


we set the associated f.p. group $G(\Phi)$ to be:

$$
\mathrm{G}(\Phi)=<a_{1}, \ldots, a_{k}, x_{1}, \ldots, x_{n} \mid w_{1}, \ldots, w_{s}>
$$

Clearly, every solution of the system $\Phi$ corresponds to a homomorphism $h: \mathrm{G}(\Phi) \rightarrow \mathrm{F}_{k}$ for which $h\left(a_{i}\right)=a_{i}$, and every such homomorphism corresponds to a solution of the system $\Phi$. Therefore, the study of sets of solutions to systems of equations in a free group is equivalent to the study of all homomorphisms from a fixed f.p. group $G$ into a free group $\mathrm{F}_{k}$ for which a given prescribed set of elements in $\mathrm{G}$ is mapped to a fixed basis of the free group $\mathrm{F}_{k}$.

Throughout this paper, this is the point of view we adapt in order to study sets of solutions to systems of equations in a free group. Since the techniques we use and the structure theory we obtain are valid for f.g. groups and not only for f.p. ones, we set $\mathrm{G}$ to be a f.g. group $\mathrm{G}=\left\langle g_{1}, \ldots, g_{m}\right\rangle, \gamma_{1}, \ldots, \gamma_{k} \in \mathrm{G}$ to be a prescribed set of elements in $\mathrm{G}$, and $\mathrm{F}_{k}$ to be a free group with a fixed basis $\left.\mathrm{F}_{k}=<a_{1}, \ldots, a_{k}\right\rangle$. With these notation our main goal is to get a structure theory for understanding the set of all homomorphisms:

$$
\operatorname{Hom}\left(\left(\mathrm{G},\left\{\gamma_{i}\right\}\right),\left(\mathrm{F}_{k},\left\{a_{i}\right\}\right)\right)=\left\{h \mid h: \mathrm{G} \rightarrow \mathrm{F}_{k}, h\left(\gamma_{i}\right)=a_{i}\right\}
$$

which for brevity we will denote $\operatorname{Hom}\left(\mathrm{G}, \mathrm{F}_{k}\right)$. We set $\mathrm{X}$ to be the Cayley graph of $\mathrm{F}_{k}$ with respect to its given basis $a_{1}, \ldots, a_{k}$. Given any homomorphism $h \in \operatorname{Hom}\left(\mathrm{G}, \mathrm{F}_{k}\right), \mathrm{G}$ admits a natural action $\lambda_{h}$ on $\mathrm{X}$ given by $\lambda_{h}(g, x)=h(g)(x)$ for every $g \in \mathrm{G}$ and $x \in \mathrm{X}$.

For presentation purposes, we start our analysis with the entire set of homomorphisms $\left\{h \mid h: \mathrm{G} \rightarrow \mathrm{F}_{k}\right\}$, without restricting the image of a finite subset of elements of the f.g. group $\mathrm{G}$ in the free group $\mathrm{F}_{k}$. As we will see in the sequel, the results we obtain for the entire (unrestricted) set of homomorphisms can be slightly modified in order to study the set of restricted homomorphisms.

Let $\left\{h_{j}\right\} \subset \operatorname{Hom}\left(\mathrm{G}, \mathrm{F}_{k}\right)$ be a set of (unrestricted) homomorphisms from $\mathrm{G}$ to $\mathrm{F}_{k}$, and suppose that the homomorphisms $\left\{h_{j}\right\}$ belong to distinct conjugacy classes (i.e., for every $j_{1}, j_{2}, 1 \leqslant j_{1}<j_{2}$, and every $f \in \mathrm{F}_{k}, f h_{j_{1}} f^{-1} \neq h_{j_{2}}$ ). For each index $j$ we fix an element $f_{j} \in \mathrm{F}_{k}$ having "minimal displacement" under the action $\lambda_{h_{j}}$ and set $\mu_{j}$ to be:

$$
\mu_{j}=\max _{1 \leqslant u \leqslant m} d_{\mathbf{X}}\left(i d ., f_{j} h_{j}\left(g_{u}\right) f_{j}^{-1}\right)=\min _{f \in \mathrm{F}_{k}} \max _{1 \leqslant u \leqslant m} d_{\mathbf{X}}\left(i d ., f h_{j}\left(g_{u}\right) f^{-1}\right) .
$$

Since the homomorphisms in the sequence $\left\{h_{j}\right\} \subset \operatorname{Hom}\left(\mathrm{G}, \mathrm{F}_{k}\right)$ are distinct, the sequence of stretching factors $\left\{\boldsymbol{\mu}_{j}\right\}$ does not contain a bounded subsequence. We set $\left\{\left(\mathrm{X}_{j}, x_{j}\right)\right\}_{j=1}^{\infty}$ to be the pointed metric spaces obtained by rescaling the metric on the Cayley graph of $\mathrm{F}_{k},(\mathrm{X}, i d$.$) , by \mu_{j} .\left(\mathrm{X}_{j}, x_{j}\right)$ is endowed with a left isometric action of our f.g. group $\mathrm{G}$ via the homomorphisms $\tau_{f_{j}} \circ h_{j}$ where $\tau_{f j}$ is the inner automorphism of $\mathrm{F}_{k}$ defined 
by $f_{j}$. This sequence of actions of $\mathrm{G}$ on the metric spaces $\left\{\left(\mathrm{X}_{j}, x_{j}\right)\right\}_{j=1}^{\infty}$ allows us to obtain an action of $\mathrm{G}$ on a real tree by passing to a Gromov-Hausdorff limit.

Proposition 1.1 ([Pa], 2.3). — Let $\left\{\mathrm{X}_{j}\right\}_{j=1}^{\infty}$ be a sequence of $\delta_{j}$-hyperbolic spaces with $\delta_{\infty}=\lim \delta_{j}=0$. Let $\mathrm{H}$ be a countable group isometrically acting on $\mathrm{X}_{j}$. Suppose there exists a base point $\overline{x_{j}}$ in $\mathrm{X}_{j}$ such that for every finite subset $\mathrm{P}$ of $\mathrm{H}$, the sets of geodesics between the images of $x_{j}$ under $\mathrm{P}$ form a sequence of totally bounded metric spaces. Then there is a subsequence converging in the Gromov topology to a $\boldsymbol{\delta}_{\infty}$-hyperbolic space $\mathrm{X}_{\infty}$ endowed with a left isometric action of $\mathrm{H}$.

Our spaces $\left\{\left(\mathrm{X}_{j}, x_{j}\right)\right\}_{j=1}^{\infty}$ endowed with the left isometric action of $\mathrm{G}$, satisfy the assumptions of the proposition and they are all trees, so they are 0-hyperbolic, hence, $\mathrm{X}_{\infty}$ is a real tree endowed with an isometric action of $\mathrm{G}$. By construction the action of $\mathrm{G}$ on the real tree $\mathrm{X}_{\infty}$ is non-trivial. Let $\left\{j_{n}\right\}_{n=1}^{\infty}$ be the subsequence for which $\left\{\left(\mathrm{X}_{j_{n}}, x_{j_{n}}\right)\right\}_{n=1}^{\infty}$ converge to the limit real tree $\mathrm{X}_{\infty}$ and let $\left(\mathrm{Y}, y_{0}\right)$ denote this (pointed) limit real tree. For convenience for the rest of this section we denote the homomorphism $f_{j_{n}} h_{j_{n}} f_{j_{n}}^{-1}: \mathrm{G} \rightarrow \mathrm{F}_{k}$ by $h_{n}$.

With the limit tree we obtained by using the Gromov-Hausdorff topology we associate natural algebraic objects, the kernel of the action of $\mathrm{G}$ on this (limit) real tree and the quotient of $\mathrm{G}$ by this kernel which we call the limit group. The first goal in our approach to the structure of sets of solutions to equations in a free group is understanding some of the basic properties of limit groups, and in particular to show they are f.p. groups and that every maximal abelian subgroup of them is a f.g. free abelian group.

Definition 1.2. - The kernel of the action of the group $\mathrm{G}$ on the limit tree $\mathrm{Y}$ is defined to be:

$$
\mathrm{K}_{\infty}=\{g \in \mathrm{G} \mid \forall y \in \mathrm{Y} g(y)=y\} .
$$

Having the kernel of the action we define the limit group to be: $\mathrm{L}_{\infty}=\mathrm{G} / \mathrm{K}_{\infty}$ and set $\eta: \mathrm{G} \rightarrow \mathrm{L}_{\infty}$ to be the natural quotient map.

The following simple facts on the kernel of the action and the limit group are important observations and will serve us throughout the rest of the paper.

Lemma 1.3. - With the notation of definition 1.2:

(i) $\mathrm{L}_{\infty}$ is a f.g. group.

(ii) If $\mathrm{Y}$ is isometric to a real line then the limit group $\mathrm{L}_{\infty}$ is f.g. free abelian.

(iii) If $g \in \mathrm{G}$ stabilizes a tripod in $\mathrm{Y}$ then for all but at most finitely many n's $g \in \operatorname{ker}\left(h_{n}\right)$ (recall that a tripod is a finite tree with 3 endpoints). In particular, if $g \in \mathrm{G}$ stabilizes a tripod then $g \in \mathrm{K}_{\infty}$. 
(iv) Let $g \in \mathrm{G}$ be an element which does not belong to $\mathrm{K}_{\infty}$. Then for all but at most finitely many $n$ 's $g \notin k e r\left(h_{n}\right)$.

(v) $\mathrm{L}_{\infty}$ is torsion-free.

(vi) Let $\left[y_{1}, y_{2}\right] \subset\left[y_{3}, y_{4}\right]$ be a pair of non-degenerate segments of $\mathrm{Y}$ and assume the stabilizer of $\left[y_{3}, y_{4}\right]$ in $\mathrm{L}_{\infty}, \operatorname{stab}\left(\left[y_{3}, y_{4}\right]\right)$, is non-trivial. Then stab $\left(\left[y_{3}, y_{4}\right]\right)$ is an abelian subgroup of $\mathrm{L}_{\infty}$ and:

$$
\operatorname{stab}\left(\left[y_{1}, y_{2}\right]\right)=\operatorname{stab}\left(\left[y_{3}, y_{4}\right]\right)
$$

Hence, the action of $\mathrm{L}_{\infty}$ on the real tree $\mathrm{Y}$ is stable.

Proof. $-\mathrm{L}_{\infty}$ is a quotient of the f.g. group $\mathrm{G}$, so it is f.g. as well. To prove (ii) note that if $\mathrm{Y}$ is isometric to a real line, then $\mathrm{L}_{\infty}$ is a f.g. $\operatorname{subgroup}$ of $\operatorname{Isom}(\mathrm{R})$. If $g \in \mathrm{G}$ preserves the orientation of $\mathrm{Y}$ and $g^{\prime} \in \mathrm{G}$ reverses it, then for large enough $n, h_{n}\left(g^{\prime}\right) h_{n}(g) h_{n}\left(g^{\prime}\right)^{-1}=h_{n}\left(g^{-1}\right)$, and since the image of the homomorphisms $h_{n}$ is a subgroup of the free group $\mathrm{F}_{k}$, necessarily $h_{n}(g)=1$ for all but finitely many $n$ 's, hence, $g \in \mathrm{K}_{\infty}$. In addition, since $g^{\prime 2}$ preserves the orientation of $\mathrm{Y}, h_{n}\left(g^{\prime 2}\right)=1$ for all but finitely many $n$ 's, so $h_{n}\left(g^{\prime}\right)=1$ for all but finitely many $n$ 's and $g^{\prime} \in \mathrm{K}_{\infty}$. Therefore, the ambient group $\mathrm{G}$ preserves the orientation of $\mathrm{Y}$, and since $\mathrm{L}_{\infty}$ is a f.g. subgroup of $\operatorname{Isom}(\mathrm{R}), \mathrm{L}_{\infty}$ is f.g. free abelian group.

To prove (iii) let $\mathrm{T}(\mathrm{A}, \mathrm{B}, \mathrm{C})$ be a tripod in $\mathrm{Y}$ and let $\mathrm{N}$ be the three valence vertex in that tripod. Let $g \in \mathrm{G}$ fix $\mathrm{T}(\mathrm{A}, \mathrm{B}, \mathrm{C})$ and let $\left(\mathrm{A}_{n}, \mathrm{~B}_{n}, \mathrm{C}_{n}\right) \subset \mathrm{X}_{n}$ be a sequence of triples of points converging into the triple $(\mathrm{A}, \mathrm{B}, \mathrm{C})$. Let $\ell=\min \left\{d_{\mathrm{Y}}(\mathrm{A}, \mathrm{N}), d_{\mathrm{Y}}(\mathrm{B}, \mathrm{N}), d_{\mathrm{Y}}(\mathrm{C}, \mathrm{N})\right\}$. From the convergence of the metric spaces $\left\{\left(\mathrm{X}_{n}, x_{n}\right)\right\}_{n=1}^{\infty}$ to the real tree $\left(\mathrm{Y}, y_{0}\right)$ we get for large enough $n$ :

$$
\max \left\{d_{\mathbf{X}_{n}}\left(\mathbf{A}_{n}, h_{n}(g)\left(\mathbf{A}_{n}\right)\right), d_{\mathbf{X}_{n}}\left(\mathbf{B}_{n}, h_{n}(g)\left(\mathbf{B}_{n}\right)\right), d_{\mathbf{X}_{n}}\left(\mathbf{C}_{n}, h_{n}(g)\left(\mathbf{C}_{n}\right)\right)\right\}<\frac{\ell}{3} .
$$

Let $\mathrm{N}_{n}$ be the center of a tripod with vertices $\mathrm{A}_{n}, \mathrm{~B}_{n}, \mathrm{C}_{n}$ in $\mathrm{X}_{n}$. By the bound on the displacement of $\mathbf{A}_{n}, \mathbf{B}_{n}, \mathbf{C}_{n}$ by $h_{n}(g)$, for large enough $n h_{n}(g)\left(\mathbf{N}_{n}\right)=\mathbf{N}_{n}$, so $h_{n}(g)=1$ for all but finitely many $n$ 's, and in particular $g \in \mathrm{K}_{\infty}$.

To prove (iv) suppose $h_{n_{v}}(g)=1$ for an infinite subsequence of indices $\left\{n_{v}\right\}$. Since the actions of $\mathrm{G}$ on the metric spaces $\left(\mathrm{X}_{n}, x_{n}\right)$ via the homomorphisms $h_{n}$ converge in the Gromov-Hausdorff topology into an action of $\mathrm{G}$ on the real tree $\mathrm{Y}$, the subsequence of actions of $\mathrm{G}$ on the metric spaces $\left(\mathrm{X}_{n_{v}}, x_{n_{v}}\right)$ via the homomorphisms $h_{n_{v}}$ converges into the same action of $\mathrm{G}$ on the real tree $\mathrm{Y}$. Since we assume $h_{n_{v}}=1$ for all $v, g$ acts trivially on the limit tree $\mathrm{Y}$, so $g \in \mathrm{K}_{\infty}$ and we may conclude part (iv) of the lemma.

To prove $\mathrm{L}_{\infty}$ is torsion-free suppose $g \in \mathrm{G}$ and $g^{p} \in \mathrm{K}_{\infty}$. Note that if the limit tree $\mathrm{Y}$ is isometric to a real line then $\mathrm{L}_{\infty}$ is a f.g. free abelian group, and in particular torsion-free. Hence, we may assume $\mathrm{Y}$ is not isometric to a real line, which implies that $g^{p}$ stabilizes a tripod in $\mathrm{Y}$ so by part (iii) of the lemma $h_{n}\left(g^{p}\right)=1$ for all but finitely 
many $n$ 's, and necessarily $h_{n}(g)=1$ for all but finitely many $n$ 's and $g \in \mathrm{K}_{\infty}$ which concludes part (v). The proof of part (vi) is identical with the proof of proposition 4.2 of [Ri-Sel].

Lemma 1.3 gives us the basic properties of the action of the limit group $\mathrm{L}_{\infty}$ on the real tree Y. These properties are essential in applying Rips' classification of (stable) actions of groups on real trees which is going to be used extensively in the sequel. In addition to the properties of the action we will need the following basic algebraic facts on the limit group $\mathrm{L}_{\infty}$.

In a free group every solvable subgroup is infinite cyclic and every maximal cyclic subgroup is malnormal. These properties are naturally inherited by the limit group $\mathrm{L}_{\infty}$.

Lemma 1.4. - With the notation of definition 1.2:

(i) Let $u_{1}, u_{2}, u_{3}$ be non-trivial elements of $\mathrm{L}_{\infty}$, and suppose that $\left[u_{1}, u_{2}\right]=1$ and $\left[u_{1}, u_{3}\right]=1$. Then $\left[u_{2}, u_{3}\right]=1$. It follows that every abelian subgroup in $\mathrm{L}_{\infty}$ is contained in a unique maximal abelian subgroup.

(ii) Every maximal abelian subgroup of $\mathrm{L}_{\infty}$ is malnormal.

(iii) Every solvable subgroup of the limit group $\mathrm{L}_{\infty}$ is abelian.

Proof. - Let $g_{1}, g_{2}, g_{3} \in \mathrm{G}$ be elements for which $\eta\left(g_{1}\right)=u_{1}, \eta\left(g_{2}\right)=u_{2}, \eta\left(g_{3}\right)=u_{3}$. Since $g_{1}, g_{2}, g_{3}$ project to non-trivial elements in $\mathrm{L}_{\infty}$, part (iv) of lemma 1.3 implies that for all but finitely many $n$ 's $h_{n}$ maps $g_{1}, g_{2}, g_{3}$ to non-trivial elements in $\mathrm{F}_{k}$. Since $\eta\left(g_{1}\right)$ commutes with both $\eta\left(g_{2}\right)$ and $\eta\left(g_{3}\right)$, part (iii) of lemma 1.3 implies that for all but finitely many $n$ 's $h_{n}\left(g_{1}\right)$ commutes with both $h_{n}\left(g_{2}\right)$ and $h_{n}\left(g_{3}\right)$. Hence, for all but finitely many $n$ 's the subgroup generated by $h_{n}\left(g_{1}\right), h_{n}\left(g_{2}\right), h_{n}\left(g_{3}\right)$ is infinite cyclic, and, in particular, $h_{n}\left(g_{2}\right)$ commutes with $h_{n}\left(g_{3}\right)$. By part (iv) of lemma $1.3 u_{2}=\eta\left(g_{2}\right)$ commutes with $u_{3}=\eta\left(g_{3}\right)$ and we get part (i) of the lemma.

To prove part (ii) let $\mathrm{A}$ be a maximal abelian subgroup of $\mathrm{L}_{\infty}$. If $\mathrm{A}$ is not malnormal there must exist elements $g_{1}, g_{2}, g \in \mathrm{G}$ so that $\eta\left(g_{1}\right), \eta\left(g_{2}\right) \in \mathrm{A}, \eta\left(g_{1}\right) \neq 1$ and $\eta(g) \eta\left(g_{1}\right) \eta\left(g^{-1}\right)=\eta\left(g_{2}\right)$. Since by part (iii) of lemma 1.3 if $r \in \mathrm{K}_{\infty}$ then for all but finitely many $n$ 's $h_{n}(r)=1$, for all but finitely many $n$ 's $h_{n}(g) h_{n}\left(g_{1}\right) h_{n}\left(g^{-1}\right)=h_{n}\left(g_{2}\right)$ and $\left[h_{n}\left(g_{1}\right), h_{n}\left(g_{2}\right)\right]=1$, so $<h_{n}\left(g_{1}\right), h_{n}\left(g_{2}\right)>$ is a cyclic subgroup of $\mathrm{F}_{k}$. Since a maximal cyclic subgroup of $\mathrm{F}_{k}$ is malnormal, for all but finitely many $n$ 's $h_{n}(g)$ commutes with both $h_{n}\left(g_{1}\right)$ and $h_{n}\left(g_{2}\right)$, so part (iv) of lemma 1.3 implies that $\eta(g)$ commutes with both $\eta\left(g_{1}\right)$ and $\eta\left(g_{2}\right)$ so $\eta(g) \in \mathrm{A}$ and $\mathrm{A}$ is malnormal.

To prove part (iii) let $\mathrm{S}$ be a solvable subgroup of $\mathrm{L}_{\infty}$. By induction we may assume that its derived subgroup $\mathrm{S}^{\prime}$ is abelian. By part (i) every (non-trivial) abelian subgroup of $\mathrm{L}_{\infty}$ is contained in a unique maximal abelian subgroup, hence, the derived 
subgroup $\mathrm{S}^{\prime}$ is contained in a (unique) maximal abelian subgroup $\mathrm{M}$ of $\mathrm{L}_{\infty}$, and by part (ii), $\mathrm{M}$ is malnormal in $\mathrm{L}_{\infty}$. Since $\mathrm{S}^{\prime}$ is normal in $\mathrm{S}$, every element $u \in \mathrm{S}$ normalizes $\mathrm{S}^{\prime}$, so $u \mathrm{M} u^{-1}$ intersects $\mathrm{M}$ non-trivially. Since $\mathrm{M}$ is malnormal, $u \in \mathrm{M}$, so $u$ commutes with $\mathrm{S}^{\prime}$. Therefore, $\mathrm{S}$ is abelian.

Proposition 1.3 shows the action of $\mathrm{L}_{\infty}$ on the real tree $\mathrm{Y}$ is stable. The original analysis of stable actions of groups on real trees applies to f.p. groups ([Be-Fel]), and the limit group $\mathrm{L}_{\infty}$ is only known to be f.g. at this point, by part (i) of lemma 1.3. Still, given the basic properties of the action of $\mathrm{L}_{\infty}$ on the real tree $\mathrm{Y}$ that we already know, we are able to apply a generalization of Rips' work to f.g. groups obtained in [Se3]. In [Se3], the real tree $\mathrm{Y}$ is divided into distinct components, where on each component a subgroup of $\mathrm{L}_{\infty}$ acts according to one of several canonical types of actions. The theorem from [Se3] we present is going to be used extensively in the next sections and its statement uses the notions and basic definitions appear in the appendix of [Ri-Sel]. Hence, we refer a reader who is not yet familiar with these notions to that appendix and to $[\mathrm{Be}-\mathrm{Fel}]$ and $[\mathrm{Be}]$.

Theorem 1.5 ([Se3], 3.1). — Let $\mathrm{G}$ be a freely indecomposable f.g. group which admits a stable isometric action on a real tree $\mathrm{Y}$. Assume the stabilizer of each tripod in $\mathrm{Y}$ is trivial.

1) There exist canonical orbits of subtrees of $\mathrm{Y}: \mathrm{Y}_{1}, \ldots, \mathrm{Y}_{k}$ with the following properties:

(i) $g \mathrm{Y}_{i}$ intersects $\mathrm{Y}_{j}$ at most in one point if $i \neq j$.

(ii) $g \mathrm{Y}_{i}$ is either identical with $\mathrm{Y}_{i}$ or it intersects it at most in one point.

(iii) The action of stab $\left(\mathrm{Y}_{i}\right)$ on $\mathrm{Y}_{i}$ is either discrete or it is of axial type or IET type.

2) $\mathrm{G}$ is the fundamental group of a graph of groups with:

(i) Vertices corresponding to orbits of branching points with non-trivial stabilizer in $\mathrm{Y}$.

(ii) Vertices corresponding to the orbits of the canonical subtrees $\mathrm{Y}_{1}, \ldots, \mathrm{Y}_{k}$ which are of axial or IET type. The groups associated with these vertices are conjugates of the stabilizers of these components. To a stabilizer of an IET component there exists an associate 2-orbifold. All boundary components and branching points in this associated 2-orbifold stabilize points in $\mathrm{Y}$. For each such stabilizer we add edges that connect the vertex stabilized by it and the vertices stabilized by its boundary components and branching points.

(iii) Edges corresponding to orbits of edges between branching points with non-trivial stabilizer in the discrete part of $\mathrm{Y}$ with edge groups which are conjugates of the stabilizers of these edges.

(iv) Edges corresponding to orbits of points of intersection between the orbits of $\mathrm{Y}_{1}, \ldots, \mathrm{Y}_{k}$.

Before concluding our preliminary study of limit groups and their action on the limit real tree, we present the following basic fact which is necessary in the sequel. 
Lemma 1.6. - If $\mathrm{L}_{\infty}$ is a limit group acting on a limit tree $\mathrm{Y}$ obtained from a converging sequence of homomorphisms from a f.g. group $\mathrm{G}$ into a free group $\mathrm{F}_{k}$ and $\mathrm{L}_{\infty}$ is freely-indecomposable, then stabilizers of non-degenerate segments which lie in the complement of the discrete parts of $\mathrm{Y}$ are trivial in $\mathrm{L}_{\infty}$. Stabilizers of segments in the discrete components of $\mathrm{Y}$ are abelian subgroups of $\mathrm{L}_{\infty}$.

Proof. - Identical to the proof of part (iii) of proposition 1.8 in [Se4].

\section{The Canonical Abelian JSJ Decomposition of a Limit Group}

In the previous section we defined limit groups and studied their basic algebraic structure. To further study the algebraic structure of a limit group $\mathrm{L}_{\infty}$, and in particular to understand what are all the possible splittings of $\mathrm{L}_{\infty}$ along abelian subgroups, we need to construct the canonical JSJ decomposition of $\mathrm{L}_{\infty}$. A canonical JSJ decomposition was first introduced in [Se2] in the case of hyperbolic groups, then generalized to cyclic splittings of f.p. groups in [Ri-Se2], and to splittings of f.p. groups over more general (slender) groups in $[\mathrm{Du}-\mathrm{Sa}]$ and $[\mathrm{Fu}-\mathrm{Pa}]$. Until this point, we only know that $\mathrm{L}_{\infty}$ is f.g. (lemma 1.3), still the JSJ theory can be applied using acylindrical accessibility [Se3] (cf. ([Se4], 2)).

To construct the JSJ decomposition of $\mathrm{L}_{\infty}$ we need to study some basic properties of abelian splittings. We first look how maximal abelian subgroups of $\mathrm{L}_{\infty}$ act on (simplicial) Bass-Serre trees corresponding to abelian splittings of $\mathrm{L}_{\infty}$. Recall that by lemma 1.4 every maximal abelian subgroup of $\mathrm{L}_{\infty}$ is malnormal.

Lemma 2.1. - Let $\mathrm{L}_{\infty}$ be a limit group, let $\mathrm{M}$ be a maximal abelian subgroup in $\mathrm{L}_{\infty}$, and let $\mathrm{A}$ be an abelian subgroup of $\mathrm{L}_{\infty}$. Then:

(i) If $\mathrm{L}_{\infty}=\mathrm{U} *_{\mathrm{A}} \mathrm{V}$ and $\mathrm{M}$ is not cyclic then $\mathrm{M}$ can be conjugated into either $\mathrm{U}$ or $\mathrm{V}$.

(ii) If $\mathrm{L}_{\infty}=\mathrm{U} *_{\mathrm{A}}$ and $\mathrm{M}$ is not cyclic then either $\mathrm{M}$ can be conjugated into $\mathrm{U}$, or $\mathrm{M}$ can be conjugated to $\mathrm{M}^{\prime}$, so that $\mathrm{L}_{\infty}=\mathrm{U} *_{\mathrm{A}} \mathrm{M}^{\prime}$.

Proof. - A non-cyclic abelian subgroup of $\mathrm{L}_{\infty}$ is elliptic in any free decomposition of $\mathrm{L}_{\infty}$, hence, we may assume that $\mathrm{A}$ is a non-trivial abelian subgroup of $\mathrm{L}_{\infty}$. To prove (i) suppose that $\mathrm{M}$ is not cyclic and $\mathrm{M}$ can not be conjugated into $\mathrm{U}$ or $\mathrm{V}$ in the amalgamated product $\mathrm{L}_{\infty}=\mathrm{U} *_{\mathrm{A}} \mathrm{V}$. Let $\mathrm{T}$ be the Bass-Serre tree corresponding to this amalgamated product. Since $\mathrm{M}$ is abelian and is not elliptic when acting on $\mathrm{T}$, it either preserves an axis or it preserves a point in the boundary of $\mathrm{T}$. If it preserves a point in the boundary of $\mathrm{T}$ and not an axis in $\mathrm{T}$, then the stabilizers of edges along a ray that approaches the point at the boundary of $\mathrm{T}$ that is preserved by $\mathrm{M}$, form an increasing sequence of abelian subgroups of $\mathrm{L}_{\infty}, \mathrm{A}_{1}<\mathrm{A}_{2}<\mathrm{A}_{3}<\ldots$, so that the union of these subgroups is $\mathrm{M}$. But all the edge groups in the Bass-Serre tree $\mathrm{T}$ are conjugates of $A$, and $M$ is malnormal in $L_{\infty}$, hence, in this case $A_{1}=A_{2}=\ldots=M$, so $\mathrm{M}$ must be elliptic. Therefore, we may assume that $\mathrm{M}$ preserves an axis in $\mathrm{T}$. 
By conjugating $\mathrm{M}$ we may assume that $\mathrm{A}$ fix an edge in the axis of $\mathrm{M}$. Since $\mathrm{M}$ is a maximal non-cyclic abelian subgroup of $\mathrm{L}_{\infty}$, and by lemma 1.4 an abelian subgroup in $\mathrm{L}_{\infty}$ is contained in a unique maximal abelian subgroup, the abelian subgroup $\mathrm{A}$ is a subgroup of $\mathrm{M}$, and $\mathrm{A}$ fixes the entire axis preserved by $\mathrm{M}$. This last observation implies that there must exist an element $u \in \mathrm{U}$ so that $u \notin \mathrm{A}$ and $u$ commutes with $\mathrm{A}$. $u$ centralizes $\mathrm{A}$, so by proposition 1.4 it must be an element of the maximal abelian subgroup containing $\mathrm{A}$, so $u \in \mathrm{M}$. But $u$ can not be an element of $\mathrm{M}$, since the only elliptic elements in $\mathrm{M}$ are the elements of $\mathrm{A}$, a contradiction.

To prove (ii) suppose $\mathrm{M}$ is non-cyclic and $\mathrm{M}$ can not be conjugated into the vertex group $\mathrm{U}$ in the abelian splitting $\mathrm{L}_{\infty}=\mathrm{U} *_{\mathrm{A}}$. Let $\mathrm{T}$ be the Bass-Serre tree corresponding to that HNN extension. $\mathrm{M}$ is abelian and not elliptic so by the argument used to prove part (i), it must preserve an axis in $\mathrm{T}$, and by conjugating $\mathrm{M}$ to $\mathbf{M}^{\prime}$, we may assume the abelian subgroup $\mathrm{A}$ fixes that entire axis of $\mathrm{M}^{\prime}$. By the argument used to prove part (i), the vertex group $\mathrm{U}$ intersects $\mathbf{M}^{\prime}$ only in the subgroup $\mathrm{A}$, so by replacing the Bass-Serre generator $t$ by $t u$ for some $u \in \mathrm{U}$ we may assume that the Bass-Serre generator $t$ is an element of $\mathbf{M}^{\prime}$. Since the Bass-Serre generator $t$ centralizes the cyclic subgroup $\mathrm{A}, \mathrm{L}_{\infty}=\mathrm{U} *_{\mathrm{A}} \mathrm{M}^{\prime}$.

By lemma 2.1 if we replace each abelian splitting of $\mathrm{L}_{\infty}$ of the form $\mathrm{L}_{\infty}=\mathrm{U} *_{\mathrm{A}}$ in which $\mathrm{A}$ is a subgroup of a non-elliptic maximal abelian subgroup $\mathrm{M}$ by the amalgamated product $\mathrm{L}_{\infty}=\mathrm{U} *_{\mathrm{A}} \mathrm{M}^{\prime}$, we get that every non-cyclic abelian subgroup of $\mathrm{L}_{\infty}$ is elliptic in all the abelian splittings under consideration. This will allow us to use acylindrical accessibility in analyzing all the abelian splittings of $\mathrm{L}_{\infty}$.

Definition 2.2 [Se3]. - A splitting of a group $\mathrm{H}$ is called $k$-acylindrical if for every element $h \in \mathrm{H}$ which is not the identity, the fixed set of $h$ when acting on the Bass-Serre tree corresponding to the splitting has diameter at most $k$.

If a limit group $\mathrm{L}_{\infty}=\mathrm{V}_{1} *_{\mathrm{A}_{1}} \mathrm{~V}_{2} *_{\mathrm{A}_{2}} \mathrm{~V}_{3} *_{\mathrm{A}_{3}} \mathrm{~V}_{4}$, where $\mathrm{A}_{1}, \mathrm{~A}_{2}, \mathrm{~A}_{3}$ are subgroups of a maximal abelian subgroup $\mathrm{M}$ that is a subgroup of $\mathrm{V}_{1}$, then one can modify the corresponding graph of groups to a tripod of groups with $V_{1}$ in the center, and $\mathrm{V}_{2}, \mathrm{~V}_{3}, \mathrm{~V}_{4}$ at the 3 roots. Since by lemma 1.4 every maximal abelian subgroup of $\mathrm{L}_{\infty}$ is malnormal, the Bass-Serre tree corresponding to this tripod of groups is 2-acylindrical. This sliding operation generalizes to an arbitrary (finite) abelian splitting of a limit group.

Lemma 2.3. - A splitting of $\mathrm{L}_{\infty}$ in which all edge groups are abelian and all noncyclic abelian groups are elliptic can always be modified (by modifying boundary monomorphisms by conjugations and sliding operations) to be 2-acylindrical. 
Lemma 2.3 shows that if in all abelian splittings of $\mathrm{L}_{\infty}$ under consideration all non-cyclic abelian subgroups are elliptic, these abelian splittings are 2-acylindrical. By lemma 2.1 if we replace abelian splittings of $\mathrm{L}_{\infty}$ having the form $\mathrm{L}_{\infty}=\mathrm{U} *_{\mathrm{A}}$ in which $\mathrm{A}$ is a subgroup of a non-elliptic maximal abelian subgroup by the amalgamated product $\mathrm{L}_{\infty}=\mathrm{U} *_{\mathrm{A}} \mathrm{M}$, we get that every non-cyclic abelian subgroup of $\mathrm{L}_{\infty}$ is elliptic in all the abelian splittings under consideration. Hence, we may assume that all abelian splittings of $\mathrm{L}_{\infty}$ under consideration are 2-acylindrical.

Following [Se2] and [Ri-Se2] in order to understand all possible splittings of $\mathrm{L}_{\infty}$ along abelian subgroups, we need to study carefully the "interaction" between any two given one-edge splittings of $\mathrm{L}_{\infty}$ over abelian subgroups.

Let $\mathrm{H}$ be a group and $\mathrm{T}$ a (simplicial) $\mathrm{H}$-tree. An element $h \in \mathrm{H}$ is either elliptic in which case it fixes a point in $\mathrm{T}$, or hyperbolic, in which case it preserves a line in $\mathrm{T}$ and acts along it as a translation. We consider two elementary splittings of $\mathrm{L}_{\infty}$ along abelian subgroups $\mathrm{H}_{\infty}=\mathrm{D}_{1} *_{\mathrm{A}_{1}} \mathrm{E}_{1}$ (or $\mathrm{L}_{\infty}=\mathrm{D}_{1} *_{\mathrm{A}_{1}}$ in which case we assume that $\mathrm{A}_{1}$ is a subgroup of a maximal abelian subgroup $\mathrm{M}$ in $\mathrm{L}_{\infty}$ and $\mathrm{M}$ is a subgroup of the vertex group $\mathrm{D}$ ), and $\mathrm{L}_{\infty}=\mathrm{D}_{2} *_{A_{2}} \mathrm{E}_{2}$ (or $\mathrm{L}_{\infty}=\mathrm{D}_{2} *_{\mathrm{A}_{2}}$ under the above conditions on $A_{2}$ ) where $A_{1}$ and $A_{2}$ are abelian subgroups. Let $T_{1}$ and $T_{2}$ be the Bass-Serre trees corresponding to the given splittings. The two given splittings are called elliptic-elliptic if $A_{1}$ is elliptic in $T_{2}$ and $A_{2}$ is elliptic in $T_{1}$, elliptic-hyperbolic if $A_{1}$ is elliptic in $T_{2}$ and $A_{2}$ is not elliptic in $T_{1}$ and hyperbolic-hyperbolic if $A_{1}$ is not elliptic in $T_{2}$ and $A_{2}$ is not elliptic in $\mathrm{T}_{1}$.

Lemma 2.1 shows that an abelian subgroup of $\mathrm{L}_{\infty}$ which is not cyclic is necessarily elliptic in all abelian splitting of $\mathrm{L}_{\infty}$ under consideration. Following [Se2] we can also exclude the case of elliptic-hyperbolic maximal abelian splittings in case $\mathrm{L}_{\infty}$ is freely indecomposable (the arguments given in [Se2] and [Ri-Se2] for cyclic splittings of groups remain valid for abelian splittings of $\mathrm{L}_{\infty}$ ).

Theorem 2.4 ([Se2], 2.2), ([Ri-Se2], 2.1). — If $\mathrm{L}_{\infty}$ is freely indecomposable then any two one-edge abelian splittings of $\mathrm{L}_{\infty}$ in which all non-cyclic abelian subgroups are elliptic, are either elliptic-elliptic or hyperbolic-hyperbolic.

Since by lemma 2.1 every abelian subgroup of $\mathrm{L}_{\infty}$ which is not cyclic is elliptic in all abelian splittings of $\mathrm{L}_{\infty}$ under consideration, pairs of hyperbolic-hyperbolic one edge splittings of $\mathrm{L}_{\infty}$ along abelian subgroups are splittings along cyclic subgroups. Hence, we may borrow the canonical quadratic decomposition constructed in [Ri-Se2] in order to understand all the hyperbolic-hyperbolic splittings of $\mathrm{L}_{\infty}$ along cyclic subgroups.

By a Z-splitting of a group we mean a splitting in which all edge groups are infinite cyclic. For the notion of a CMQ (canonical maximal QH) subgroup, and a weakly essential s.c.c. on a 2-orbifold we refer the reader to section 4 of [Ri-Se2]. 
Theorem 2.5 ([Ri-Se2], 5.6). - Let $\mathrm{H}$ be a f.g. group with a single end which is not a 2-orbifold group. There exists a (canonical) reduced Z-splitting of $\mathrm{H}$ which we call the quadratic decomposition of $\mathrm{H}$ with the following properties:

(i) Every canonical maximal $\mathrm{QH}$ subgroup (GMQ) of $\mathrm{H}$ is conjugate to a vertex group in the quadratic decomposition. In particular, there are only finitely many conjugacy classes of GMQ subgroups. Every edge group is a cyclic boundary subgroup of one of the CMQ subgroups, and every vertex with a non-CMQ vertex group is adjacent only to vertices stabilized by $\mathrm{CMQ}$ subgroups in the canonical quadratic decomposition.

(ii) An elementary Z-splitting $\mathrm{H}=\mathrm{A} *_{\mathrm{C}} \mathrm{B}$ or $\mathrm{H}=\mathrm{A} *_{\mathrm{C}}$ which is hyperbolic in another elementary $\mathrm{Z}$-splitting is obtained from the quadratic decomposition of $\mathrm{H}$ by cutting a 2-orbifold corresponding to a $\mathrm{CMQ}$ subgroup of $\mathrm{H}$ along a weakly essential s.c.c.

(iii) The edge group of any elementary $\mathrm{Z}$-splitting $\mathrm{H}=\mathrm{A} *_{\mathrm{C}} \mathrm{B}$ or $\mathrm{H}=\mathrm{A} *_{\mathrm{C}}$ can be conjugated into a vertex group of the quadratic decomposition. In case it can be conjugated into a vertex group which is not a CMQ subgroup, the given elementary Z-splitting is elliptic-elliptic with respect to any other elementary $\mathrm{Z}$-splitting of $\mathrm{H}$.

(iv) The quadratic decomposition of $\mathrm{H}$ is unique up to sliding, conjugation and modifying boundary monomorphisms by conjugation (see section 1 of [Ri-Se2] for the definition of these notions).

To construct the JSJ decomposition of $\mathrm{L}_{\infty}$ we need to further refine the quadratic decomposition to include the set of elliptic-elliptic abelian decompositions. In general, such refinement can not be obtained for a f.g. group as was shown by M. Dunwoody [Du]. Fortunately, the abelian splittings of $\mathrm{L}_{\infty}$ we consider are 2-acylindrical according to lemma 2.3, which allows us to apply acylindrical accessibility in order to complete the construction of the JSJ decomposition.

Theorem 2.6 ([Se3], $[\mathrm{We}])$. — Let $\mathrm{H}$ be a f.g. freely indecomposable group. For a given $k$ there exists an integer $\lambda(k, \mathrm{H})$ so that the number of vertices and edges in all $k$-acylindrical splittings of $\mathrm{H}$ does not exceed $\lambda(k, \mathrm{H})$.

(Note that in [We], the bound $\lambda(k, \mathrm{H})$ is given explicitly.) To construct the abelian JSJ decomposition of a freely indecomposable limit group $\mathrm{L}_{\infty}$, we start with the collection of all cyclic splittings of $\mathrm{L}_{\infty}$ in which every non-cyclic abelian subgroup is elliptic, from which we construct the cyclic JSJ decomposition of $\mathrm{L}_{\infty}$. To construct this cyclic JSJ decomposition of $\mathrm{L}_{\infty}$, we start with the quadratic decomposition of $\mathrm{L}_{\infty}$, and successively refine the quadratic decomposition using all the cyclic splittings of $\mathrm{L}_{\infty}$ for which their (cyclic) edge groups are elliptic in the quadratic decomposition, and in which every non-cyclic abelian subgroup of $\mathrm{L}_{\infty}$ is elliptic (see section 7 of [Ri-Se2] for a description of this refinement procedure). In the construction of the cyclic JSJ decomposition for f.p. groups presented in [Ri-Se2], this refinement procedure is shown 
to terminate using the generalized accessibility of M. Bestvina and M. Feighn [Be-Fe2]. The limit group $\mathrm{L}_{\infty}$ is not known to be f.p. so generalized accessibility can not be applied. However, acylindrical accessibility (theorem 2.6) guarantees the termination of the refinement procedure, from which we obtain the cyclic JSJ decomposition of the limit group $\mathrm{L}_{\infty}$ (note that at this point we do not know if the cyclic JSJ decomposition of $\mathrm{L}_{\infty}$ is trivial or not).

Having the cyclic JSJ decomposition of $\mathrm{L}_{\infty}$, we construct the abelian JSJ decomposition of $\mathrm{L}_{\infty}$ by successively refining the non- $\mathrm{QH}$, non-abelian vertex groups in the cyclic JSJ decomposition using the collection of non-cyclic abelian decompositions of $\mathrm{L}_{\infty}$. Again, acylindrical accessibility (theorem 2.6) guarantees that this refinement procedure terminates, and we finally obtain the abelian JSJ decomposition of a freely indecomposable limit group. Note that since a limit group admits a faithful stable action on a real tree, in which segment stabilizers are abelian and stabilizers of tripods are trivial (lemma 1.3), theorem 1.5 implies that if $\mathrm{L}_{\infty}$ is non-cyclic it admits a nontrivial abelian splitting in which all non-cyclic abelian subgroups are elliptic. Hence, if $\mathrm{L}_{\infty}$ is not a surface group or an abelian group, its abelian JSJ decomposition is necessarily non-trivial.

Theorem 2.7 (cf. [Ri-Se2], 7.1). — Suppose $\mathrm{L}_{\infty}$ is a freely indecomposable limit group. There exists a reduced unfolded splitting of $\mathrm{L}_{\infty}$ with abelian edge groups, which we call an abelian JSJ (Jaco-Shalen-Fohannson) decomposition of $\mathrm{L}_{\infty}$ with the following properties:

(i) Every canonical maximal $\mathrm{QH}$ subgroup (CMQ of $\mathrm{L}_{\infty}$ is conjugate to a vertex group in the JSJ decomposition. Every $\mathrm{QH}$ subgroup of $\mathrm{L}_{\infty}$ can be conjugated into one of the GMQ subgroups of $\mathrm{L}_{\infty}$. Every vertex group in the JSJ decomposition which is not a GMQ subgroup of $\mathrm{L}_{\infty}$ is elliptic in any abelian splitting of $\mathrm{L}_{\infty}$ under consideration.

(ii) A one edge abelian splitting $\mathrm{L}_{\infty}=\mathrm{D} *_{\mathrm{A}} \mathrm{E}$ or $\mathrm{H}_{\infty}=\mathrm{D} *_{\mathrm{A}}$ under consideration which is hyperbolic in another elementary abelian splitting is obtained from the abelian JSJ decomposition of $\mathrm{L}_{\infty}$ by cutting a 2-orbifold corresponding to a $\mathrm{CMQ}$ subgroup of $\mathrm{L}_{\infty}$ along a weakly essential s.c.c.

(iii) Let $\Theta$ be a one edge splitting along an abelian subgroup $\mathrm{L}_{\infty}=\mathrm{D} *_{\mathrm{A}} \mathrm{E}$ or $\mathrm{L}_{\infty}=\mathrm{D} *_{\mathrm{A}}$ under consideration, which is elliptic with respect to any other one edge abelian splitting of $\mathrm{L}_{\infty}$ under consideration. Then $\Theta$ is obtained from the JSJ decomposition of $\mathrm{L}_{\infty}$ by a sequence of collapsings, foldings, and conjugations.

(iv) If $\mathrm{JSJ}_{1}$ is another JSJ decomposition of $\mathrm{L}_{\infty}$, then $\mathrm{JSJ}_{1}$ is obtained from the JSJ decomposition by a sequence of slidings, conjugations and modifying boundary monomorphisms by conjugations (see section 1 of [Ri-Se2] for these notions).

Theorem 2.7 gives us the canonical abelian JSJ decomposition of $\mathrm{L}_{\infty}$ which essentially describes all the abelian splittings of $\mathrm{L}_{\infty}$ along abelian subgroups. This decomposition is a basic tool in our approach to obtaining a structure theory for sets of solutions of equations in a free group. 


\section{The Ganonical Gyclic JSJ Decomposition of a Limit Group}

In section 2 we have constructed the (canonical) abelian JSJ decomposition of a limit group $\mathrm{L}_{\infty}$, which includes (in an appropriate sense) all abelian splittings of $\mathrm{L}_{\infty}$. To analyze sets of solutions to systems of equations in a free group we will be interested not in all abelian splittings but rather in cyclic ones. Theorem 1.5 shows that if $\mathrm{L}_{\infty}$ is freely indecomposable and not abelian it must admit a non-trivial abelian splitting, hence, in case it is not abelian nor a surface group it's abelian JSJ decomposition is not the trivial one. In this section our goal is to prove analogous statements for cyclic splittings of $\mathrm{L}_{\infty}$, i.e., we prove that if $\mathrm{L}_{\infty}$ is freely indecomposable and not abelian it must admit a cyclic splitting, and we define it's non-trivial canonical cyclic JSJ decomposition. We prove the existence of a cyclic splitting of $\mathrm{L}_{\infty}$ by showing that if the JSJ decomposition of $\mathrm{L}_{\infty}$ contains only non-cyclic abelian edge groups, one may further refine the abelian JSJ decomposition of $\mathrm{L}_{\infty}$, which clearly contradicts the canonical properties of the JSJ (theorem 2.7).

With the notation of the first section let $\mathrm{G}$ be a f.g. group, let $\left\{h_{n} \mid h_{n}: \mathrm{G} \rightarrow \mathrm{F}_{k}\right\}$ be a convergent sequence of homomorphisms, and let $\mathrm{L}_{\infty}$ be the corresponding limit group and $\left(\mathrm{Y}, y_{0}\right)$ the corresponding limit tree.

Definition 3.1. - We say that a cyclic splitting of a limit group, $\mathrm{L}_{\infty}=\mathrm{A} *_{\mathrm{Z}} \mathrm{B}$ or $\mathrm{L}_{\infty}=\mathrm{A} *_{\mathrm{Z}}$, is essential, if both vertex groups $\mathrm{A}$ and $\mathrm{B}$ are not cyclic. We say that it is principal if it is essential, and either $\mathrm{A}$ is abelian or the centralizer of the edge group $\mathrm{Z}$ in $\mathrm{L}_{\infty}$ is cyclic.

Theorem 3.2. — Let $\mathrm{L}_{\infty}$ be a non-abelian and freely-indecomposable limit group. Then $\mathrm{L}_{\infty}$ admits a principal cyclic splitting.

Proof. - Since $\mathrm{L}_{\infty}$ is freely indecomposable it admits a canonical abelian JSJ decomposition by theorem 2.7. Note that since every abelian subgroup in $\mathrm{L}_{\infty}$ is contained in a unique maximal abelian subgroup by lemma 1.4, all vertex groups which are adjacent to an abelian vertex group in the abelian JSJ decomposition of $\mathrm{L}_{\infty}$ are non-abelian. We construct an abelian splitting $\Lambda_{\mathrm{L}_{\infty}}$ of $\mathrm{L}_{\infty}$ in the following way. If the abelian JSJ decomposition of $\mathrm{L}_{\infty}$ contains no cyclic vertex groups we set $\Lambda_{\mathrm{L}_{\infty}}$ to be the abelian JSJ decomposition. Otherwise, for each cyclic vertex group in the abelian JSJ decomposition we collapse an edge which connects the cyclic vertex group to a neighbor. We set $\Lambda_{\mathrm{L}_{\infty}}$ to be the obtained abelian decomposition. Since $\mathrm{L}_{\infty}$ is a limit group, i.e., it is obtained from a convergent sequence of homomorphisms $\left\{h_{n} \mid h_{n}: \mathrm{G} \rightarrow \mathrm{F}_{k}\right\}, \Lambda_{\mathrm{L}_{\infty}}$ is a non-trivial abelian decomposition. Indeed, from the sequence of homomorphisms $\left\{h_{n}\right\}$ we can extract a subsequence that converges to an action of $\mathrm{L}_{\infty}$ on some real tree Y. From this action, we get a graph of groups $\Theta$ with abelian edge groups and fundamental group $\mathrm{L}_{\infty}$. Since the real tree $\mathrm{Y}$ was obtained from a sequence of homomorphisms $\left\{h_{n} \mid h_{n}: \mathrm{G} \rightarrow \mathrm{F}_{k}\right\}$, if $\mathrm{C}$ is a cyclic subgroup of $\mathrm{L}_{\infty}, \mathrm{C}$ fixes 
a point in $\mathrm{Y}$, and if a non-trivial subgroup $\mathrm{C}^{\prime}<\mathrm{C}$ fixes a non-degenerate segment in $\mathrm{Y}$, then $\mathrm{C}$ fixes that segment as well. Hence, if $\mathrm{L}_{\infty}$ inherits a cyclic splitting from its action on the real tree $\mathrm{Y}$, then this cyclic splitting is essential. Therefore, there exists either a non-cyclic abelian splitting of $\mathrm{L}_{\infty}$, or an essential cyclic splitting of $\mathrm{L}_{\infty}$, so $\Lambda_{\mathrm{L}_{\infty}}$ is non-trivial.

Clearly, if $\Lambda_{\mathrm{L}_{\infty}}$ contains an edge with cyclic stabilizer, $\mathrm{L}_{\infty}$ admits an essential cyclic splitting. If the centralizer of this edge stabilizer is also cyclic, $\mathrm{L}_{\infty}$ admits a principal cyclic splitting. Hence, we may assume that the centralizers of all edge groups in $\Lambda_{\mathrm{L}_{\infty}}$ are abelian and non-cyclic, and that $\Lambda_{\mathrm{L}_{\infty}}$ contains no vertex with an abelian non-cyclic vertex group A so that all edge stabilizers connected to this vertex generate a cyclic subgroup in $\mathrm{A}$.

Recall that if $\Delta$ is a (finite) graph of groups with fundamental group $\mathrm{H}$, and $\mathrm{T}$ is a maximal subtree in the graph of groups $\Delta$, then $\Delta$ is generated by the vertex groups in the maximal tree $\mathrm{T}$, and elements that correspond to edges in $\Delta$ that are not in the maximal tree $\mathrm{T}$, that are called Bass-Serre generators.

Let $g_{1}, \ldots, g_{q}$ be a set of generators of G. Clearly, $\mathrm{L}_{\infty}=\left\langle\eta\left(g_{1}\right), \ldots, \eta\left(g_{q}\right)\right\rangle$. We fix a maximal subtree of the graph of groups $\Lambda_{\mathrm{L}_{\infty}}$, set $\mathrm{V}_{\infty}^{1}, \ldots, \mathrm{V}_{\infty}^{m}$ to be the vertex groups in that maximal subtree, $\mathrm{E}_{\infty}^{1}, \ldots, \mathrm{E}_{\infty}^{s}$ to be the (abelian) edge groups in $\Lambda_{\mathrm{L}_{\infty}}$, and $t_{\infty}^{1}, \ldots, t_{\infty}^{b}$ to be the set of Bass-Serre generators in $\Lambda_{\mathrm{L}_{\infty}}$ with respect to our fixed maximal subtree. Since we assume that $\mathrm{L}_{\infty}$ admits no principal cyclic splitting, the centralizers of all the edge groups $\mathrm{E}_{\infty}^{j}$ are abelian and non-cyclic and, in particular, the abelian JSJ decomposition of $\mathrm{L}_{\infty}$ contains no CMQ subgroups.

Let $v_{1}^{1}, \ldots, v_{\ell_{1}}^{1}, \ldots, v_{1}^{m}, \ldots, v_{\ell_{m}}^{m}, t_{1}, \ldots, t_{b}$ be elements in $\mathrm{G}$ for which $\eta\left(v_{1}^{i}\right), \ldots, \eta\left(v_{\ell_{i}}^{i}\right) \in \mathbf{V}_{\infty}^{i}$ for every $1 \leqslant i \leqslant m, \eta\left(t_{j}\right)=t_{\infty}^{j}$ and for every generator $g_{j}$ of $\mathrm{G}$ :

$$
g_{j}=w_{j}\left(v_{1}^{1}, \ldots, v_{\ell_{m}}^{m}, t_{1}, \ldots, t_{b}\right) .
$$

Clearly, we may assume that each of the vertex groups $\mathrm{V}_{\infty}^{i}$ is generated by $\eta\left(v_{1}^{i}\right), \ldots, \eta\left(v_{\ell_{i}}^{i}\right)$ and the edge groups $\mathrm{E}_{\infty}^{j}$ connected to the vertex stabilized by $\mathrm{V}_{\infty}^{i}$ in $\Lambda_{\mathrm{L}_{\infty}}$. For each $j$ let $e_{1}^{j}, e_{2}^{j}, \ldots \in \mathrm{G}$ be a set of elements for which $\eta\left(e_{p}^{j}\right) \in \mathrm{E}_{\infty}^{j}$ and in addition $\eta\left(e_{1}^{j}\right), \eta\left(e_{2}^{j}\right), \ldots$ generate the edge group $\mathrm{E}_{\infty}^{j}$.

Our strategy in proving theorem 3.2 is to show that if $\Lambda_{\mathrm{L}_{\infty}}$ contains no principal cyclic splitting, then the abelian JSJ decomposition of the limit group $\mathrm{L}_{\infty}$ can be further refined, which clearly contradicts its canonical properties. If we could have assumed that all the vertex groups in the graph of groups $\Lambda_{\mathrm{L}_{\infty}}$ are f.g. then since a f.g. subgroup of a limit group is a limit group, each of the vertex groups of $\Lambda_{\mathrm{L}_{\infty}}$ is a limit group. Hence, every non-abelian vertex group in $\Lambda_{\mathrm{L}_{\infty}}$ admits a non-trivial abelian JSJ decomposition, and the abelian edge groups of $\Lambda_{\mathrm{L}_{\infty}}$, that are all assume to have non-cyclic centralizers, are elliptic in these abelian JSJ decompositions. Therefore, the abelian JSJ decompositions of the non-abelian vertex groups in $\Lambda_{\mathrm{L}_{\infty}}$ can be used to 
construct a proper refinement of the graph of groups $\Lambda_{\mathrm{L}_{\infty}}$, that gives rise to a proper refinement of the abelian JSJ decomposition of $\mathrm{L}_{\infty}$.

Unfortunately, the edge groups in the abelian JSJ decomposition of $\Lambda_{\mathrm{L}_{\infty}}$ may not be f.g. and so are the vertex groups in $\Lambda_{\mathrm{L}_{\infty}}$. To construct a proper refinement of the abelian JSJ decomposition of $\mathrm{L}_{\infty}$ in the presence of vertex groups that may not be f.g. we apply the shortening argument presented in section 3 of [Se4] for proving the Hopf property of hyperbolic groups. Using the shortening argument we construct an action of a quotient of the limit group $\mathrm{L}_{\infty}, \mathrm{Q}_{\infty}$, on some real tree $\mathrm{R}$, where the vertex groups in the given abelian JSJ decomposition of $\mathrm{L}_{\infty}$ are embedded into $\mathrm{Q}_{\infty}$, and at least one of those vertex groups act non-trivially on the real tree $\mathrm{R}$. This gives us an abelian decomposition of that vertex group, that can be used to obtain a proper refinement of the given abelian JSJ decomposition of the original limit group $\mathrm{L}_{\infty}$.

The shortening argument we apply is rather involved technically because we can not assume that vertex groups are finitely generated. A reader who is not familiar with the shortening argument may choose to look first at an excellent presentation of the shortening argument in case the vertex groups are f.g., that appears in M. Bestvina's survey paper on R-trees ([Be], 7.4).

Our goal is to construct a proper refinement of the abelian JSJ decomposition of $\mathrm{L}_{\infty}$. We start by defining a sequence of finitely presented groups $\left\{\mathrm{U}_{n}\right\}$ together with homomorphisms $\tau_{n}: \mathrm{U}_{n-1} \rightarrow \mathrm{U}_{n}$ which approximate the f.g. group $\mathrm{L}_{\infty}$. The groups $\mathrm{U}_{n}$ admit homomorphisms into $\mathrm{F}_{k}$, and the abelian decomposition $\Lambda_{\mathrm{L}_{\infty}}$ of $\mathrm{L}_{\infty}$ can be "lifted" to (f.g. abelian) decompositions of each of the $\mathrm{U}_{n}$ 's. This lifting property of the $\mathrm{U}_{n}$ 's is crucial in applying our shortening argument ([Se3], [Ri-Se1]) for obtaining further refinements of the abelian JSJ decompositions of $\mathrm{L}_{\infty}$ in case $\mathrm{L}_{\infty}$ does not admit a principal cyclic splitting, and was first introduced in proving the Hopf property for hyperbolic groups [Se4].

We define the groups $\mathrm{U}_{n}$ iteratively. We set $\mathrm{U}_{1}$ to be a free group generated by the elements:

$$
\mathrm{U}_{1}=<x_{1}^{1}, \ldots, x_{\ell_{1}}^{1}, \ldots, x_{1}^{m}, \ldots, x_{\ell_{m}}^{m}, y_{1}, \ldots, y_{b}, z_{1}^{1}, \ldots, z_{1}^{s}>
$$

Clearly, the homomorphism $h_{1}: \mathrm{G} \rightarrow \mathrm{F}_{k}$ lifts to a homomorphism $\lambda_{1}: \mathrm{U}_{1} \rightarrow \mathrm{F}_{k}$ by setting: $\lambda_{1}\left(x_{p}^{i}\right)=h_{1}\left(v_{p}^{i}\right), \lambda_{1}\left(y_{r}\right)=h_{1}\left(t_{r}\right)$ and $\lambda_{1}\left(z_{1}^{j}\right)=h_{1}\left(e_{1}^{j}\right)$. We define $\mathrm{U}_{2}$ to be the group generated by:

$$
\mathrm{U}_{2}=<x_{1}^{1}, \ldots, x_{\ell_{1}}^{1}, \ldots, x_{1}^{m}, \ldots, x_{\ell_{m}}^{m}, y_{1}, \ldots, y_{b}, z_{1}^{1}, \ldots, z_{1}^{s}, z_{2}^{1}, \ldots, z_{2}^{s}>
$$

together with the relations $\left[z_{1}^{j}, z_{2}^{j}\right]=1$ for $j=1, \ldots, s$. Clearly, there exists a natural homomorphism $\tau_{1}: \mathrm{U}_{1} \rightarrow \mathrm{U}_{2}$. By part (iii) of lemma 1.3, for $a_{2}$ large enough the homomorphism $h_{a_{2}}: \mathrm{G} \rightarrow \mathrm{F}_{k}$ lifts to a homomorphism $\lambda_{2}: \mathrm{U}_{2} \rightarrow \mathrm{F}_{k}$ by setting: $\lambda_{2}\left(x_{p}^{i}\right)=h_{a_{2}}\left(v_{p}^{i}\right), \lambda_{2}\left(y_{r}\right)=h_{a_{2}}\left(t_{r}\right), \lambda_{2}\left(z_{1}^{j}\right)=h_{a_{2}}\left(e_{1}^{j}\right)$ and $\lambda_{2}\left(z_{2}^{j}\right)=h_{a_{2}}\left(e_{2}^{j}\right)$. 
Defining $\mathrm{U}_{1}$ and $\mathrm{U}_{2}$ we continue by defining the groups $\mathrm{U}_{n}$, the homomorphisms $\tau_{n}$ and the homomorphisms $\lambda_{n}$ iteratively. We first define the group $\mathrm{H}_{n}$ to be the group generated by:

$$
\mathrm{H}_{n}=<x_{1}^{1}, \ldots, x_{\ell_{1}}^{1}, \ldots, x_{1}^{m}, \ldots, x_{\ell_{m}}^{m}, y_{1}, \ldots, y_{b}, z_{1}^{1}, \ldots, z_{1}^{s}, \ldots, z_{n}^{1}, \ldots, z_{n}^{s}>
$$

together with the relations $\left[z_{p_{1}}^{j}, z_{p_{2}}^{j}\right]=1$ for $j=1, \ldots, s$ and $p_{1}, p_{2}=1, \ldots, n$. The group $\mathrm{H}_{n}$ admits a natural epimorphism $\sigma_{n}$ onto $\mathrm{L}_{\infty}$ defined by setting $\sigma_{n}\left(x_{p}^{i}\right)=\eta\left(v_{p}^{i}\right), \sigma_{n}\left(y_{r}\right)=t_{\infty}^{r}$, and $\sigma_{n}\left(z_{d}^{j}\right)=\eta\left(e_{d}^{j}\right)$. We define the group $\mathrm{U}_{n}$ to be a quotient of the group $\mathrm{H}_{n}$. To the existing set of relations of $\mathrm{H}_{n}$ we add all words $w$ in the given defining generators of $\mathrm{H}_{n}$ for which $\sigma_{n}(w)=1$, the length of $w$ in the defining generators of $\mathrm{H}_{n}$ is at most $n$, and all the generators appear in the word $w$ are mapped by $\sigma_{n}$ into the same vertex group $\mathrm{V}_{\infty}^{i}$ in the abelian decomposition $\Lambda_{\mathrm{L}_{\infty}}$ of $\mathrm{L}_{\infty}$.

Clearly, there exists a natural map $\tau_{n}: \mathrm{U}_{n-1} \rightarrow \mathrm{U}_{n}$, and by part (iii) of lemma 1.3, there exists some integer $a_{n}>a_{n-1}$ so that the homomorphism $h_{n}: \mathrm{G} \rightarrow \mathrm{F}_{k}$ lifts to a homomorphism $\lambda_{n}: \mathrm{U}_{n} \rightarrow \mathrm{F}_{k}$ defined by: $\lambda_{n}\left(x_{p}^{i}\right)=h_{a_{n}}\left(v_{p}^{i}\right), \lambda_{n}\left(y_{r}\right)=h_{a_{n}}\left(t_{r}\right)$, and $\lambda_{n}\left(z_{d}^{j}\right)=h_{a_{n}}\left(e_{d}^{j}\right)$.

Since the second set of defining relations of the group $\mathrm{U}_{n}$ are all words in generators which are mapped by $\sigma_{n}$ into the same vertex group in $\Lambda_{\mathrm{L}_{\infty}}$, each of the groups $\mathrm{U}_{n}$ admits an abelian splitting $\Lambda_{n}$ which projects by $\sigma_{n}$ into the abelian decomposition $\Lambda_{\mathrm{L}_{\infty}}$ of $\mathrm{L}_{\infty}$, i.e., each of the vertex groups $\mathrm{V}_{n}^{i}$ in $\Lambda_{n}$ satisfies $\sigma_{n}\left(\mathrm{~V}_{n}^{i}\right)<\mathrm{V}_{\infty}^{i}$, each of the edge groups $\mathrm{E}_{n}^{j}=<z_{1}^{j}, \ldots, z_{n}^{j}>$ satisfies $\sigma_{n}\left(\mathrm{E}_{n}^{j}\right)<\mathrm{E}_{\infty}^{j}$, and each of the BassSerre generators in $\Lambda_{n}$ satisfies $\sigma_{n}\left(\mathcal{\psi}_{r}\right)=t_{\infty}^{r}$. We will denote by $\operatorname{Mod}\left(\mathbf{U}_{n}\right)$ the subgroup of $\operatorname{Aut}\left(\mathrm{U}_{n}\right)$ generated by inner automorphisms and Dehn twists along edges of $\Lambda_{n}$. We set $\mathrm{W}_{n}$ to be the subgroup of $\mathrm{U}_{n}$ generated by the $x_{p}^{i}$ s $\mathrm{s}$ and the $y_{r}$ 's. Clearly, the homomorphism $\tau_{n}: \mathrm{U}_{n-1} \rightarrow \mathrm{U}_{n}$ restricts to an epimorphism from $\mathrm{W}_{n-1}$ onto $\mathrm{W}_{n}$, and $\lambda_{n}$ restricts to a homomorphism from $\mathrm{W}_{n}$ into $\mathrm{F}_{k}$. Defining the groups $\mathrm{U}_{n}$ 's and $\mathrm{W}_{n}$ 's and the homomorphisms between them we have obtained the following diagram:

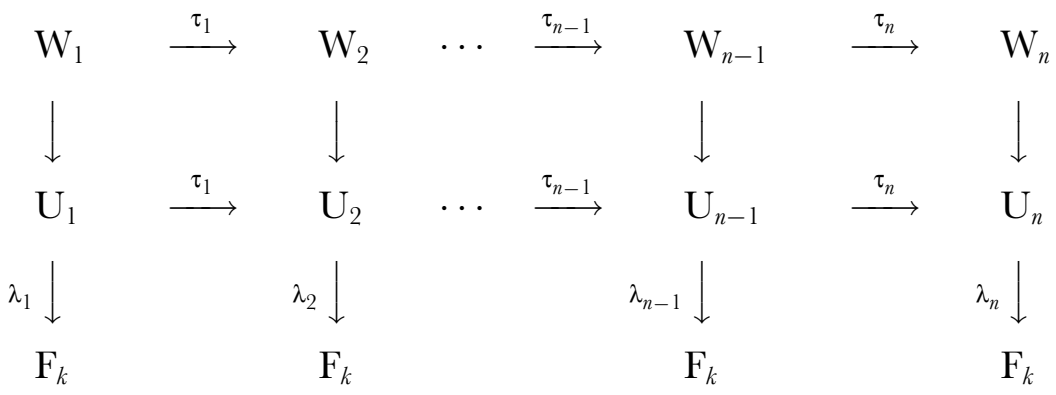

where the direct limit of the $\left\{\mathrm{W}_{n}\right\}$ 's and the maps $\tau_{n}$ 's is the limit group $\mathrm{L}_{\infty}$.

At this stage we are finally ready to modify the shortening argument of ([Se3], [Ri-Sel]) in order to further refine the abelian JSJ decomposition of $\mathrm{L}_{\infty}$ in case $\mathrm{L}_{\infty}$ 
does not admit a principal cyclic splitting. Let $\mathrm{T}$ be a maximal tree in $\Lambda_{\mathrm{L}_{\infty}}$. We may order the $m$ vertices $q_{i}$ in $\mathrm{T}$, so that $q_{1}$ is connected to $q_{2}, q_{3}$ is connected to the subtree of $\mathrm{T}$ spanned by $q_{1}$ and $q_{2}$, and in general $q_{p}$ is connected to the subtree of $\mathrm{T}$ spanned by the pre-chosen vertices $q_{1}, q_{2}, \ldots, q_{p-1}$.

Without loss of generality we may assume that this is the original order defined on the vertex groups $\mathrm{V}_{\infty}^{i}$ of the abelian decomposition $\Lambda_{\mathrm{L}_{\infty}}$ of $\mathrm{L}_{\infty}$. Let $\mathrm{X}$ be the Cayley graph of the free group $\mathrm{F}_{k}$ with respect to a (fixed) finite set of generators, and let $d_{\mathrm{X}}$ be the standard simplicial metric on $\mathrm{X}$. For each element $f \in \mathrm{F}_{k}$, each positive integer $n$, and each automorphism $\varphi \in \operatorname{Mod}\left(\mathbf{U}_{n}\right)$ we define the following (stretching) constants:

$$
\begin{aligned}
& \mu_{i}(n, f, \varphi)=\max \left(d_{\mathrm{X}}\left(i d ., f \lambda_{n}\left(\varphi\left(x_{1}^{i}\right)\right) f^{-1}\right), \ldots, d_{\mathrm{X}}\left(i d ., f \lambda_{n}\left(\varphi\left(x_{\ell_{i}}^{i}\right)\right) f^{-1}\right)\right) \\
& \chi_{r}(n, f, \varphi)=d_{\mathrm{X}}\left(i d ., f \lambda_{n}\left(\varphi\left(y_{r}\right)\right) f^{-1}\right)
\end{aligned}
$$

and the corresponding $(m+b)$-tuple:

$$
\operatorname{tup}(n, f, \varphi)=\left(\mu_{1}(n, f, \varphi), \ldots, \mu_{m}(n, f, \varphi), \chi_{1}(n, f, \varphi), \ldots, \chi_{b}(n, f, \varphi)\right) .
$$

On the set of $(m+b)$-tuples we define the natural lexicographical order, and for each $n$ we choose $f_{n} \in \mathrm{F}_{k}$ and $\varphi_{n} \in \operatorname{Mod}\left(\mathbf{U}_{n}\right)$ for which $\operatorname{tup}\left(n, f_{n}, \boldsymbol{\varphi}_{n}\right)$ is a minimal $(m+b)$-tuple in the set $\{\operatorname{tup}(n, f, \varphi)\}$ with respect to the lexicographical order. We set $d i s p_{n}$ to be the sum of the elements in the $(m+b)$-tuple $\operatorname{tup}\left(n, f_{n}, \varphi_{n}\right)$.

If $w_{1}, w_{2} \in \mathrm{W}_{1}$ are a pair of elements for which $\sigma_{1}\left(w_{1}\right), \sigma_{1}\left(w_{2}\right) \in \mathrm{V}_{\infty}^{i}$ for some $i$, then for some index $n_{0}$ and for every $n>n_{0}, \tau_{n} \circ \ldots \circ \tau_{0}\left(w_{1}\right)$ and $\tau_{n} \circ \ldots \circ \tau_{0}\left(w_{2}\right)$ belong to the same (i-th) vertex group $\mathrm{V}_{n}^{i}$ in the (f.g. abelian) splitting $\Lambda_{n}$ of $\mathrm{U}_{n}$. Hence, for every $n>n_{0}$ both $\tau_{n} \circ \ldots \circ \tau_{0}\left(w_{1}\right)$ and $\tau_{n} \circ \ldots \circ \tau_{0}\left(w_{2}\right)$ are being mapped to their (same) conjugates by every modular automorphism $\varphi \in \operatorname{Mod}\left(\mathbf{U}_{n}\right)$, and in particular, by the chosen one $\varphi_{n}$. Since in addition at least one of the vertex groups $\mathrm{V}_{\infty}^{i}$ contains abelian non-cyclic subgroups, there could not exist a sequence of indices $n_{1}<n_{2}<\ldots$ and corresponding elements $f_{n_{j}} \in \mathrm{F}_{k}$ so that for every index $j, f_{n_{j}}$ conjugates $\lambda_{n_{j}} \circ \varphi_{n_{j}}\left(x_{p}^{i}\right)$ into $\lambda_{n_{1}} \circ \varphi_{n_{1}}\left(x_{p}^{i}\right)$ for $i=1, \ldots, m$ and $p=1, \ldots, \ell_{i}$ and conjugates $\lambda_{n_{j}} \circ \varphi_{n_{j}}\left(y_{r}\right)$ into $\lambda_{n_{1}} \circ \varphi_{n_{1}}\left(y_{r}\right)$ for every $r=1, \ldots, b$. Therefore, in particular, the sequence of displacement constants $\left\{d i s p_{n}\right\}$ does not contain a bounded subsequence, i.e., dis $p_{n} \rightarrow \infty$.

The groups $\mathrm{W}_{n}$ admit a natural action on the Cayley graph $\mathrm{X}$ of $\mathrm{F}_{k}$ which we denote $\rho_{n}: \mathrm{W}_{n} \times \mathrm{X} \rightarrow \mathrm{X}$, by setting $\rho_{n}(w, x)=\lambda_{n} \circ \varphi_{n}(w)(x)$ for every $w \in \mathrm{W}_{n}$ and $x \in \mathrm{X}$. Since the sequence of displacements $\left\{d i s p_{n}\right\}$ is not bounded, and the group $\mathrm{W}_{n}$ is a natural quotient of the group $\mathrm{W}_{1}$, we may rescale the metric on the Cayley graph $\mathrm{X}$ by dis $p_{n}$, and apply proposition $1.1([\mathrm{~Pa}], 2.3)$ for the sequence of actions $\left\{\rho_{n}\right\}$ to obtain a subsequence (still denoted $\left.\left\{\rho_{n}\right\}\right)$ converging into a (pointed) real tree $\left(\mathrm{R}, r_{0}\right)$ equipped with a non-trivial isometric action of $\mathrm{W}_{1}$. We set $\mathrm{KW}_{\infty}$ to be the kernel of the action of $\mathrm{W}_{1}$ on the real tree $\mathrm{R}$, and the f.g. group $\mathrm{Q}_{\infty}$ to be the quotient 
$\mathrm{Q}_{\infty}=\mathrm{W}_{1} / \mathrm{KW}_{\infty} . \mathrm{Q}_{\infty}$ is also a limit group so it satisfies lemmas $1.2,1.3$ and 1.4 and its action on the pointed limit tree $\left(\mathrm{R}, r_{0}\right)$ satisfies the properties given in theorem 1.5.

$\mathrm{L}_{\infty}$ is the direct limit of the groups $\left\{\mathrm{W}_{n}\right\}$ and the epimorphisms $\left\{\tau_{n}\right\}$ between them. Since if $w_{1}, w_{2} \in W_{0}$ are a pair of elements for which $\eta \circ \lambda_{1}\left(w_{1}\right), \eta \circ \lambda_{1}\left(w_{2}\right) \in V_{\infty}^{i}$ for some $i$, then for some index $n_{0}$ and for every $n>n_{0}, \tau_{n} \circ \ldots \circ \tau_{0}\left(w_{1}\right)$ and $\tau_{n} \circ \ldots \circ \tau_{0}\left(w_{2}\right)$ belong to the same (i-th) vertex group $\mathrm{V}_{n}^{i}$ in the (f.g. abelian) splitting $\Lambda_{n}$ of $\mathrm{U}_{n}$, for every $n>n_{0}$ both $\tau_{n} \circ \ldots \circ \tau_{0}\left(w_{1}\right)$ and $\tau_{n} \circ \ldots \circ \tau_{0}\left(w_{2}\right)$ are being mapped to their (same) conjugates by the chosen automorphism $\varphi_{n}$. Hence, $\mathrm{V}_{\infty}^{i}$ is naturally embedded in the group $Q_{\infty}$. By our construction, $Q_{\infty}$ is generated by the subgroups $\mathrm{V}_{\infty}^{i}$ and the images of the elements $y_{r} \in \mathrm{W}_{1}$ in $\mathrm{Q}_{\infty}$.

Proposition 3.3. - If all the subgroups $\mathrm{V}_{\infty}^{1}, \ldots, \mathrm{V}_{\infty}^{m}$ (the vertex groups in the abelian decomposition $\Lambda_{\mathrm{L}_{\infty}}$ of $\left.\mathrm{L}_{\infty}\right)$ fix points in the pointed real tree $\left(\mathrm{R}, r_{0}\right)$, then they all fix the base point $r_{0} \in \mathrm{R}$.

Proof. - By our assumptions $\mathrm{V}_{\infty}^{1}$ fixes a point $r \in \mathrm{R}$. For fixed index $n$, the conjugating elements $\left\{f_{n}\right\}$ and the modular automorphisms $\left\{\boldsymbol{\varphi}_{n}\right\}$ were chosen to minimize the $(m+b)$-tuple tup $(n, f, \varphi)$, so in particular for fixed $n$, the chosen elements have to minimize the stretching constant $\mu_{1}(n, f, \varphi)$.

If $\mathrm{V}_{\infty}^{1}$ does not fix the base point $r_{0} \in \mathrm{R}$, then for some $n_{0}$ and all $n>n_{0}$ it is possible to find elements $h_{n} \in \mathrm{F}_{k}$ for which:

$$
\max _{1 \leqslant p \leqslant \ell_{1}} d_{\mathrm{X}}\left(i d ., h_{n} f_{n} \boldsymbol{\lambda}_{n}\left(\boldsymbol{\varphi}_{n}\left(x_{p}^{1}\right)\right) f_{n}^{-1} h_{n}^{-1}\right)<\max _{1 \leqslant p \leqslant \ell_{1}} d_{\mathrm{X}}\left(i d ., f_{n} \boldsymbol{\lambda}_{n}\left(\boldsymbol{\varphi}_{n}\left(x_{p}^{1}\right)\right) f_{n}^{-1}\right) .
$$

Hence, $\mu_{1}\left(n, h_{n} f_{n}, \varphi_{n}\right)<\mu_{1}\left(n, f_{n}, \varphi_{n}\right)$, which implies tup $\left(n, h_{n} f_{n}, \varphi_{n}\right)<\operatorname{tup}\left(n, f_{n}, \varphi_{n}\right)$, a contradiction to the choice of the pair $f_{n}, \varphi_{n}$. Therefore, $\mathrm{V}_{\infty}^{1}$ fixes the base point $r_{0} \in \mathrm{R}$.

$\mathrm{V}_{\infty}^{1}$ fixes the base point $r_{0} \in \mathrm{R}$, so if the claim of proposition 3.2 does not hold there must exist a minimal index $i_{0}$, so that $\mathrm{V}_{\infty}^{1}, \ldots, \mathrm{V}_{\infty}^{i_{0}-1}$ fix the base point $r_{0} \in \mathrm{R}$ whereas $\mathrm{V}_{\infty}^{i_{0}}$ does not fix $r_{0} \in \mathrm{R}$. To prove the proposition we adapt the shortening argument of [Se3] and [Ri-Sel] to show that in this last case it is possible to find a sequence of modular automorphisms $\left\{\boldsymbol{\alpha}_{n} \in \operatorname{Mod}\left(\mathbf{U}_{n}\right)\right\}$ so that for some index $n_{0}$ and every $n>n_{0}$ :

$$
\operatorname{tup}\left(n, f_{n}, \varphi_{n} \circ \alpha_{n}\right)<\operatorname{tup}\left(n, f_{n}, \varphi_{n}\right)
$$

Lemma 3.4. - If $\mathrm{V}_{\infty}^{1}, \ldots, \mathrm{V}_{\infty}^{i_{0}-1}$ fix the base point $r_{0} \in \mathrm{R}$ and $\mathrm{V}_{\infty}^{i_{0}}$ does not fix the point $r_{0}$, then there exists some index $n_{0}$ so that for all $n>n_{0}$ there exist modular automorphisms $\alpha_{n} \in \operatorname{Mod}\left(\mathbf{U}_{n}\right)$ for which: 
(i) $\alpha_{n}\left(\mathrm{~V}_{n}^{i}\right)=\mathrm{V}_{n}^{i}$ for $i=1, \ldots, i_{0}-1$.

(ii) $\mu_{i_{0}}\left(n, f_{n}, \varphi_{n} \circ \alpha_{n}\right)<\mu_{i_{0}}\left(n, f_{n}, \varphi_{n}\right)$.

Proof. - Identical to the proof of theorem 6.10 in [Ri-Sel].

By lemma 3.4, if $i_{0}$ is the first index for which $\mathrm{V}_{\infty}^{i_{0}}$ does not fix the base point $r_{0} \in \mathrm{R}$, then for some index $n_{0}$ and all $n>n_{0}$ it is possible to find modular automorphisms $\alpha_{n} \in \operatorname{Mod}\left(\mathrm{U}_{n}\right)$ for which:

(1) $\mu_{i}\left(n, f_{n}, \varphi_{n} \circ \alpha_{n}\right)=\mu_{i}\left(n, f_{n}, \varphi_{n}\right)$ for $i=1, \ldots, i_{0}-1$

(2) $\mu_{i_{0}}\left(n, f_{n}, \varphi_{n} \circ \alpha_{n}\right)<\mu_{i_{0}}\left(n, f_{n}, \varphi_{n}\right)$.

Hence, $\operatorname{tup}\left(n, f_{n}, \varphi_{n} \circ \alpha_{n}\right)<\operatorname{tup}\left(n, f_{n}, \varphi_{n}\right)$ in the lexicographical order on the $(m+b)$ tuples tup $(n, f, \varphi)$, which clearly contradicts the way the pair $\left(f_{n}, \boldsymbol{\varphi}_{n}\right)$ were chosen. Therefore, all the groups $\mathrm{V}_{\infty}^{i}$ have to fix the base point $r_{0} \in \mathrm{R}$ and the proof of proposition 3.3 is concluded.

Showing that all vertex groups $\mathrm{V}_{\infty}^{i}$ fix the base point $r_{0} \in \mathrm{R}$, we continue by showing that the images of the elements $y_{1}, \ldots, y_{b}$ in $Q_{\infty}$ fix $r_{0}$ as well. Since $Q_{\infty}$ is generated by the $\mathrm{V}_{\infty}^{i}$ 's and the images of the $y_{j}$ 's, we will get that the entire group $\mathrm{Q}_{\infty}$ fixes the base point $r_{0}$, a contradiction to the non-triviality of the action of $\mathrm{Q}_{\infty}$ on the real tree $\mathrm{R}$, hence, at least one of the groups $\mathrm{V}_{\infty}^{1}, \ldots, \mathrm{V}_{\infty}^{m}$ does not fix a point while acting on the real tree $\mathrm{R}$.

Lemma 3.5. - Suppose that not all the elements $y_{1}, \ldots, y_{b}$ fix the base point $r_{0}$, and let $j_{0}$ be the minimal index for which $y_{j_{0}}$ does not fix the base point $r_{0} \in \mathrm{R}$. Then there exists some index $n_{0}$ so that for all $n>n_{0}$ there exist modular automorphisms $\beta_{n} \in \operatorname{Mod}\left(\mathbf{U}_{n}\right)$ for which:

(i) $\beta_{n}\left(\mathrm{~V}_{n}^{i}\right)=\mathrm{V}_{n}^{i}$ for $i=1, \ldots, m$.

(ii) $\beta_{n}\left(y_{j}\right)=y_{j}$ for $j=1, \ldots, j_{0}-1$.

(iii) $\chi_{j_{0}}\left(n, f_{n}, \varphi_{n} \circ \beta_{n}\right)<\chi_{j_{0}}\left(n, f_{n}, \varphi_{n}\right)$.

Proof. - Identical to the proof of theorem 6.13 in [Ri-Sel].

By lemma 3.5 if $j_{0}$ is the first index for which $y_{j_{0}}$ does not fix the base point $r_{0} \in \mathrm{R}$, then for some index $n_{0}$ and all $n>n_{0}$ it is possible to find modular automorphisms $\beta_{n} \in \operatorname{Mod}\left(\mathrm{U}_{n}\right)$ for which:

(1) $\mu_{i}\left(n, f_{n}, \varphi_{n} \circ \beta_{n}\right)=\mu_{i}\left(n, f_{n}, \varphi_{n}\right)$ for $i=1, \ldots, m$

(2) $\chi_{j}\left(n, f_{n}, \varphi_{n} \circ \beta_{n}\right)=\chi_{j}\left(n, f_{n}, \varphi_{n}\right)$ for $j=1, \ldots, j_{0}-1$

(3) $\chi_{j_{0}}\left(n, f_{n}, \varphi_{n} \circ \beta_{n}\right)<\chi_{j_{0}}\left(n, f_{n}, \varphi_{n}\right)$.

Hence, $\operatorname{tup}\left(n, f_{n}, \varphi_{n} \circ \beta_{n}\right)<\operatorname{tup}\left(n, f_{n}, \varphi_{n}\right)$ in the lexicographical order on the $(m+b)$-tuples $\operatorname{tup}(n, f, \varphi)$, which clearly contradicts the way the pair $\left(f_{n}, \varphi_{n}\right)$ were chosen. Therefore, 
if all the groups $\mathrm{V}_{\infty}^{1}, \ldots, \mathrm{V}_{\infty}^{m}$ fix points in the real tree $\left(\mathrm{R}, r_{0}\right)$ then all the groups $\mathrm{V}_{\infty}^{i}$ and all the elements $y_{j}$ have to fix the base point $r_{0} \in \mathrm{R}$. Since $\mathrm{Q}_{\infty}$ is generated by the groups $\mathrm{V}_{\infty}^{i}$ and the elements $y_{j}$, the entire group $\mathbf{Q}_{\infty}$ fixes the base point $r_{0} \in \mathrm{R}$. This clearly contradicts the non-triviality of the action of $Q_{\infty}$ on the real tree $\mathrm{R}$, and therefore:

Proposition 3.6. - At least one of the vertex groups in the abelian decomposition $\Lambda_{\mathrm{L}_{\infty}}$ of $\mathrm{L}_{\infty}, \mathrm{V}_{\infty}^{1}, \ldots, \mathrm{V}_{\infty}^{m}$, acts non-trivially on the pointed real tree $\left(\mathrm{R}, r_{0}\right)$.

Since the limit group $\mathrm{L}_{\infty}$ was assumed non-abelian, and since by lemma 1.4 every abelian subgroup of $\mathrm{L}_{\infty}$ is contained in a unique maximal abelian subgroup and every maximal abelian subgroup is malnormal, not all vertex groups in $\Lambda_{\mathrm{L}_{\infty}}$, $\mathrm{V}_{\infty}^{1}, \ldots, \mathrm{V}_{\infty}^{m}$ are abelian.

Lemma 3.7. - Let $\mathrm{A}$ be an abelian subgroup of one of the groups $\mathrm{V}_{\infty}^{i}$. Then either $\mathrm{A}$ fixes a point in the real tree $\mathrm{R}$, or $\mathrm{A}$ can be written as a direct sum $\mathrm{A}=\widehat{\mathrm{A}}+<a_{1}, \ldots, a_{c}>$ where $<a_{1}, \ldots, a_{c}>\simeq \mathrm{Z}^{c}, \widehat{\mathrm{A}}$ fixes a point in the real tree $\mathrm{R}$ and no non-trivial element $\left.a \in<a_{1}, \ldots, a_{c}\right\rangle$ fixes a point in $\mathrm{R}$.

Proof. - The lemma is obvious if A is cyclic, so suppose A is non-cyclic. Since $\mathrm{A}<\mathrm{V}_{\infty}^{i}$ it is naturally embedded in $\mathrm{Q}_{\infty}$. If $\mathrm{Q}_{\infty}$ is freely decomposable, $\mathrm{A}$ is a subgroup of one of the freely-indecomposable factors of $Q_{\infty}, \widehat{Q}_{\infty}$. The action of $\widehat{Q}_{\infty}$ on the real tree $\left(\mathrm{R}, r_{0}\right)$ can be analyzed using theorem 1.5 ([Se3], 3.1). It follows from that theorem that an abelian subgroup can either fix a point in $\left(\mathrm{R}, r_{0}\right)$, preserve an axial component in $\left(\mathrm{R}, r_{0}\right)$, or fix an axis of one of its elements and this axis is not an axial component in $\left(\mathbf{R}, r_{0}\right)$.

If A fixes an axial component, then A can be expressed as a subgroup Stab $<$ A that pointwise stabilizes this component, direct sum with a f.g. free abelian subgroup of A, where this f.g. free abelian subgroup acts faithfully on the axial component preserved by $\mathrm{A}$. If $\mathrm{A}$ preserves a line in $\left(\mathrm{R}, r_{0}\right)$ which is not an axial component, $\mathrm{A}$ can be expressed as a subgroup Stab $<$ A which fixes this line direct sum with a cyclic subgroup $\mathrm{C}$ of $\mathrm{A}$ that acts discretely on the line preserved by $\mathrm{A}$.

Proposition 3.6 proves that at least one of the vertex groups $\mathrm{V}_{\infty}^{i}$ acts nontrivially on the limit tree $\left(\mathrm{R}, r_{0}\right)$. To get a contradiction, we show that if the abelian JSJ decomposition of $\mathrm{L}_{\infty}$ contains no principal cyclic splittings, then each of the nonabelian vertex groups $\mathrm{V}_{\infty}^{i}$ admits a fixed point when acting on the limit tree $\left(\mathrm{R}, r_{0}\right)$.

Proposition 3.8. - If $\Lambda_{\mathrm{L}_{\infty}}$ contains no principal cyclic splittings, then every non-abelian vertex group $\mathrm{V}_{\infty}^{i}$ in $\Lambda_{\mathrm{L}_{\infty}}$ fixes a point $r_{i} \in \mathrm{R}$ when acting on the real tree $\left(\mathrm{R}, r_{0}\right)$. 
Proof. - Suppose $\mathrm{V}_{\infty}^{i}$ is not abelian and it does not fix a point in the real tree $\left(\mathrm{R}, r_{0}\right) . \mathrm{V}_{\infty}^{i}$ is generated by the abelian edge groups $\mathrm{E}_{\infty}^{j}$ together with the finite set of elements $v_{1}^{i}, \ldots, v_{\ell_{1}}^{i}$. Hence, by lemma $3.7, \mathrm{~V}_{\infty}^{i}$ is generated by finitely many abelian subgroups $\mathrm{A}_{1}^{i}, \ldots, \mathrm{A}_{u}^{i}$ that admit fix points while acting on $\left(\mathrm{R}, r_{0}\right)$ together with a finite set of elements $v_{1}^{i}, \ldots, v_{\ell_{1}^{\prime}}^{i}$. Therefore, by theorem $1.5 \mathrm{~V}_{\infty}^{i}$ inherits a finite graph of groups from its action on $\left(\mathrm{R}, r_{0}\right)$, a graph of groups which is non-trivial and in which all edge stabilizers are abelian.

By lemma 2.1 we can modify this finite graph of groups to get a non-trivial abelian splitting $\Gamma^{i}$ of $\mathrm{V}_{\infty}^{i}$ in which all non-cyclic maximal abelian subgroups are elliptic, and by theorem 1.5 if $\Gamma^{i}$ contains a cyclic edge group then $V_{\infty}^{i}$ admits a principal cyclic splitting in which all non-cyclic maximal abelian subgroups are elliptic. Now, we can properly refine the abelian decomposition $\Lambda_{\infty}^{i}$ by replacing the vertex stabilized by $\mathrm{V}_{\infty}^{i}$ with $\Gamma^{i}$. If $\Gamma^{i}$ contains a cyclic edge group, $\mathrm{L}_{\infty}$ admits a principal cyclic splitting and the theorem follows. Otherwise, the obtained proper refinement can not be obtained from the abelian JSJ decomposition of $\mathrm{L}_{\infty}$ by a sequence of foldings, collapsings, slidings, and modifying boundary monomorphisms which clearly contradicts the canonical properties of the abelian JSJ decomposition of $\mathrm{L}_{\infty}$ (theorem 2.7) and the proposition follows.

Since by proposition 3.8 all non-abelian vertex groups $\mathrm{V}_{\infty}^{i}$ in the abelian decomposition $\Lambda_{\mathrm{L}_{\infty}}$ are elliptic when acting on the real tree $\left(\mathrm{R}, r_{0}\right)$, and since by proposition 3.6 not all the vertex groups $\mathrm{V}_{\infty}^{1}, \ldots, \mathrm{V}_{\infty}^{m}$ fix points in $\left(\mathrm{R}, r_{0}\right)$, there must exist at least one abelian vertex group $\mathrm{V}_{\infty}^{i_{0}}$ that does not fix a point in $\left(\mathrm{R}, r_{0}\right)$. By lemma $3.7 \mathrm{~V}_{\infty}^{i_{0}}=\mathrm{A}^{i_{0}}+\mathrm{D}^{i_{0}}$ where $\mathrm{A}^{i_{0}}$ fixes a point in the real tree $\left(\mathbf{R}, r_{0}\right)$ and $\mathrm{D}^{i_{0}}$ is a f.g. free abelian group that acts faithfully on the line preserved by $\mathrm{V}_{\infty}^{i_{0}}$.

Since every maximal abelian subgroup in $\mathrm{L}_{\infty}$ is malnormal, all adjacent vertex groups to an abelian vertex group in $\Lambda_{\mathrm{L}_{\infty}}$ are non-abelian. Since all non-abelian vertex groups fix points in $\left(\mathbf{R}, r_{0}\right)$ all edge groups connected to $\mathrm{V}_{\infty}^{i_{0}}$ are subgroups of $\mathrm{A}^{i_{0}}$. If $\mathrm{A}^{i_{0}}$ is an infinite cyclic subgroup of $\mathrm{V}_{\infty}^{i_{0}}$ we have found a principal cyclic splitting of the limit group $\mathrm{L}_{\infty}$, hence, we may assume that $\mathrm{A}^{i_{0}}$ is non-cyclic.

We set $\mathrm{L}_{\infty}^{1}$ to be the fundamental group of the graph of groups obtained from $\Lambda_{\mathrm{L}_{\infty}}$ by replacing the vertex group $\mathrm{V}_{\infty}^{i_{0}}$ with its subgroup $\mathrm{A}^{i_{0}}$. Clearly, $\mathrm{L}_{\infty}^{1}$ is a subgroups of $\mathrm{L}_{\infty}$, and since $\mathrm{V}_{\infty}^{i_{0}}=\mathrm{A}^{i_{0}}+\mathrm{D}^{i_{0}}, \mathrm{~L}_{\infty}^{1}$ is also a quotient of $\mathrm{L}_{\infty}$. Hence, $\mathrm{L}_{\infty}^{1}$ is f.g. as well, and the first Betti number of $\mathrm{L}_{\infty}^{1}$ is strictly smaller than that of $\mathrm{L}_{\infty}: b_{1}\left(\mathrm{~L}_{\infty}^{1}\right)<b_{1}\left(\mathrm{~L}_{\infty}\right)$. If $\mathrm{L}_{\infty}^{1}$ is freely decomposable, then the abelian subgroup $\mathrm{A}^{i_{0}}$ can be conjugated into one of the free factors, so necessarily $\mathrm{L}_{\infty}$ must be freely decomposable as well, a contradiction to our assumptions. Hence, we may conclude that $\mathrm{L}_{\infty}^{1}$ is freely indecomposable.

$\mathrm{L}_{\infty}^{1}$ is also a limit group, so we may repeat the shortening argument for $\mathrm{L}_{\infty}^{1}$ and the abelian splitting it inherits from the abelian decomposition $\Lambda_{\mathrm{L}_{\infty}}$ of $\mathrm{L}_{\infty}$ to obtain a 
new limit group $\mathrm{Q}_{\infty}^{1}$ acting on a new real tree $\left(\mathrm{R}^{1}, r_{0}^{1}\right)$. By propositions 3.3-3.8 either one of the non-abelian groups $\mathrm{V}_{\infty}^{i}$ acts non-trivially on the new limit tree $\left(\mathrm{R}^{1}, r_{0}^{1}\right)$, in which case it is either possible to obtain a principal cyclic decomposition of $\mathrm{L}_{\infty}$ or to refine the abelian JSJ decomposition of $\mathrm{L}_{\infty}$ which contradicts its canonical properties (theorem 2.7), or we obtain a new limit group $\mathrm{L}_{\infty}^{2}$ which is a subgroup and a quotient of $\mathrm{L}_{\infty}^{1}$, and $b_{1}\left(\mathrm{~L}_{\infty}^{2}\right)<b_{1}\left(\mathrm{~L}_{\infty}^{1}\right)<b_{1}\left(\mathrm{~L}_{\infty}\right)$. Therefore, after repeating this process finitely many times (depending on the first Betti number of $\mathrm{L}_{\infty}$ ) we must obtain a non-trivial abelian splitting of one of the non-abelian groups $\mathrm{V}_{\infty}^{i}$, which allows us to either obtain a principal cyclic decomposition of $\mathrm{L}_{\infty}$ or to refine the abelian JSJ decomposition of $\mathrm{L}_{\infty}$ and contradict its canonical properties. This finally concludes the proof of theorem 3.2 .

Theorem 3.2 proves the existence of a principal cyclic splitting of a freelyindecomposable non-abelian limit group. In [Ri-Se2] a canonical (cyclic) JSJ decomposition that "encodes" all cyclic splittings of a f.p. freely-indecomposable group is being constructed. Since all cyclic splittings can be modified to be 2-acylindrical, we may replace the generalized accessibility of $\mathrm{M}$. Bestvina and M. Feighn [Be-Fe2] used in the construction of the cyclic JSJ decomposition of f.p. groups in [Ri-Se2] by acylindrical accessibility (theorem 2.6) to obtain the canonical cyclic decomposition of a limit group. The cyclic JSJ decomposition is obtained by collapsing all the edges with non-cyclic edge groups in the abelian JSJ decomposition of a limit group. Theorem 3.2 shows it is non-trivial in case the limit group is not abelian.

Theorem 3.9 (cf. ([Ri-Se2], 7.1). — Suppose $\mathrm{L}_{\infty}$ is freely indecomposable. There exists a reduced unfolded $\mathrm{Z}$-splitting of $\mathrm{L}_{\infty}$, which we call the cyclic JSJ (Jaco-Shalen-Fohannson) decomposition of $\mathrm{L}_{\infty}$ with the following properties:

(i) Every canonical maximal $\mathrm{QH}$ subgroup (CMQ) of $\mathrm{L}_{\infty}$ is conjugate to a vertex group in the JSJ decomposition. Every $\mathrm{QH}$ subgroup of $\mathrm{L}_{\infty}$ can be conjugated into one of the GMQ subgroups of $\mathrm{L}_{\infty}$. Every vertex group in the cyclic JSJ decomposition which is not a CMQ subgroup of $\mathrm{L}_{\infty}$ is elliptic in any $\mathrm{Z}$-splitting of $\mathrm{L}_{\infty}$ under consideration.

(ii) A one edge Z-splitting $\mathrm{L}_{\infty}=\mathrm{D} *_{\mathrm{Z}} \mathrm{E}$ or $\mathrm{H}_{\infty}=\mathrm{D} *_{\mathrm{Z}}$ under consideration which is hyperbolic in another elementary Z-splitting is obtained from the cyclic JSJ decomposition of $\mathrm{L}_{\infty}$ by cutting a 2-orbifold corresponding to a $\mathrm{CMQ}$ subgroup of $\mathrm{L}_{\infty}$ along a weakly essential s.c.c.

(iii) Let $\Theta$ be a one edge Z-splitting $\mathrm{L}_{\infty}=\mathrm{D} *_{\mathrm{Z}} \mathrm{E}$ or $\mathrm{L}_{\infty}=\mathrm{D} *_{\mathrm{Z}}$, which is elliptic with respect to any other one edge $\mathrm{Z}$-splitting of $\mathrm{L}_{\infty}$. Then $\Theta$ is obtained from the cyclic JSJ decomposition of $\mathrm{L}_{\infty}$ by a sequence of collapsings, foldings, and conjugations.

(iv) Let $\Lambda$ be a general Z-splitting of $\mathrm{L}_{\infty}$. There exists a $\mathrm{Z}$-splitting $\Lambda_{1}$ obtained from the cyclic JSJ decomposition by splitting the CMQ subgroups along weakly essential s.c.c. on their corresponding 2-orbifolds, so that there exists a $\mathrm{L}_{\infty}$-equivariant simplicial map between a subdivision of the Bass-Serre tree $\mathrm{T}_{\Lambda_{1}}$ to $\mathrm{T}_{\Lambda}$. 
(v) If $\mathrm{JSJ}_{1}$ is another cyclic JSJ decomposition of $\mathrm{L}_{\infty}$, then $\mathrm{JSJ}_{1}$ is obtained from the JSJ decomposition by a sequence of slidings, conjugations and modifying boundary monomorphisms by conjugations (see section 1 of [Ri-Se2] for these notions).

(vi) If $\mathrm{L}_{\infty}$ is non-abelian then the cyclic JSJ decomposition of $\mathrm{L}_{\infty}$ is non-trivial. Furthermore, the cyclic JSJ decomposition can be collapsed to give a principal cyclic splitting of $\mathrm{L}_{\infty}$ in this case.

\section{Finite Presentability of Limit Groups and $\omega$-Residually Free Groups}

In the first section we defined limit groups and studied the basic properties of their action on the corresponding limit tree. Since a limit group is a quotient of a f.g. group, it is naturally f.g. as well. To get a structure theory for sets of solutions of equations in a free group, we will have to show limit groups are f.p. and not only f.g. This will imply, in particular, that a limit group is a $\omega$-residually free group (definition 4.5 below).

To study some of the basic algebraic properties of limit groups we prove that limits groups are strongly accessible. Strong accessibility for certain classes of splittings of f.p. groups was recently proved by T. Delzant and L. Potyagailo [De-Po]. However, limit groups are assumed to be f.g. and we still do not know they are f.p. so the results of [De-Po] can not be applied. To prove that limit groups are strongly accessible, we prove an analogue of a Haken hierarchy for a limit group, and associate with such a group a canonical analysis lattice, from which some basic algebraic properties of limit groups can be deduced.

Let $\mathrm{L}_{\infty}$ be a limit group. We construct the analysis lattice associated with $\mathrm{L}_{\infty}$ as follows. At the 0 -th level of the hierarchy we place $\mathrm{L}^{0}=\mathrm{L}_{\infty}$. If $\mathrm{L}_{\infty}$ is either a free group, a surface group, or an abelian group this 0 -th level is the entire analysis lattice. Otherwise, we first factor $\mathrm{L}^{0}=\mathrm{L}_{\infty}$ into freely indecomposable groups, then in each factor which is not abelian or a surface group, we define the groups in the 1-st level of the analysis lattice of $\mathrm{L}_{\infty}, \mathrm{L}_{1}^{1}, \ldots, \mathrm{L}_{m_{1}}^{1}$ to be the vertex groups in the canonical cyclic JSJ decompositions of the freely indecomposable factors of $\mathrm{L}_{\infty}$. Note that since $\mathrm{L}_{\infty}$ is f.g., the groups $\mathrm{L}_{i}^{1}$ are f.g. as well, hence, they are also limit groups.

To define the next levels we continue iteratively. If a group in the $i$-th level $\mathrm{L}_{j}^{i}$ is either a free group, a surface group or an abelian group we don't continue to the $i+1$-st level from this branch. Otherwise, we first factor $\mathrm{L}_{j}^{i}$ into freely indecomposable factors, and then define the successive groups in the $i+1$-level to be the vertex groups in canonical JSJ decompositions of the freely indecomposable factors of $\mathrm{L}_{j}^{i}$.

Our main technical goal in this section is to show that the construction of the analysis lattice of a limit group terminates. To do that we will show that the complexity of limit groups that appear in higher levels in the lattice is smaller than the complexity 
of limit groups in lower levels. To measure the complexity of the limit groups $\mathrm{L}_{j}^{i}$ we will use their first Betti number which we denote $b_{1}$.

Theorem 4.1. - Let $\mathrm{L}_{\infty}$ be a limit group. The construction of the analysis lattice of $\mathrm{L}_{\infty}$ terminates after finitely many steps.

Proof. - Since we are going to use the first Betti number to measure the complexity of a limit group, our first step in obtaining theorem 4.1 is showing that the first Betti number of a non-cyclic limit group is at least 2.

Lemma 4.2. - Let $\mathrm{G}$ be a f.g. group, let $h_{n}$ be a convergent sequence of homomorphisms of $\mathrm{G}$ into $\mathrm{F}_{k}$, and let $\mathrm{L}_{\infty}$ be the corresponding limit group and $\mathrm{Y}$ the corresponding limit tree. If $\mathrm{L}_{\infty}$ is non-cyclic then $b_{1}\left(\mathrm{~L}_{\infty}\right) \geqslant 2$.

Proof. - We may assume $\mathrm{L}_{\infty}$ is non-abelian. The image of the homomorphisms $h_{n}: \mathrm{G} \rightarrow \mathrm{F}_{k}$ is a subgroup of the free group $\mathrm{F}_{k}$. If there exists a sequence of indices for which the image is cyclic, then by part (iv) of lemma $1.3 \mathrm{~L}_{\infty}$ is abelian, which contradicts our assumptions. Hence, except for at most finitely many $n$ 's the image of the homomorphisms $h_{n}$ is a non-abelian free subgroup of $\mathrm{F}_{k}$.

The limit group $\mathrm{L}_{\infty}$ is f.g. and a quotient of the f.g. group $G$, so let $\mathrm{L}_{\infty}=\mathrm{G} /<r_{1}, r_{2}, \ldots,>$. We denote by $\mathrm{G}_{i}$ the group $\mathrm{G}_{i}=\mathrm{G} /<r_{1}, \ldots, r_{i}>$. Clearly there is a canonical epimorphism from $\mathrm{G}_{i}$ onto $\mathrm{G}_{i+1}$, and the direct limit of the groups $\left\{\mathrm{G}_{i}\right\}$ is $\mathrm{L}_{\infty}$. Since for each fixed index $i$, for all but finitely many $n$ 's, the homomorphisms $h_{n}: \mathrm{G} \rightarrow \mathrm{F}_{k}$ split through $\mathrm{G}_{i}$, i.e., they can be written as a composition $h_{n}=f_{n}^{i} \circ p^{i}$ where $p^{i}$ is the canonical quotient map from $\mathrm{G}$ onto $\mathrm{G}_{i}$, and $f_{n}^{i}$ is a homomorphism from $\mathrm{G}_{i}$ into $\mathrm{F}_{k}, \mathrm{G}_{i}$ admits a non-abelian free quotient, so $b_{1}\left(\mathrm{G}_{i}\right) \geqslant 2$. Since $\mathrm{L}_{\infty}$ is the direct limit of the groups $\mathrm{G}_{i}$ and the canonical epimorphisms between them:

$$
b_{1}(\mathrm{G}) \geqslant b_{1}\left(\mathrm{G}_{1}\right) \geqslant b_{1}\left(\mathrm{G}_{2}\right) \geqslant \ldots \geqslant b_{1}\left(\mathrm{G}_{i}\right) \geqslant \ldots \geqslant b_{1}\left(\mathrm{~L}_{\infty}\right) .
$$

If $A_{1}, A_{2}, A_{3}, \ldots$ is a sequence of f.g. abelian groups so that there exist epimorphisms from $\mathrm{A}_{s}$ to $\mathrm{A}_{s+1}$ for every $s$, then there must exist some $s_{0}$ for which $\mathrm{A}_{s_{0}} \simeq \mathrm{A}_{s_{0}+1} \simeq \mathrm{A}_{s_{0}+2} \simeq \ldots$. Applying that for the first homology groups of the $\mathrm{G}_{i}$ 's it follows that there exists some index $i_{0}$ for which:

$$
b_{1}\left(\mathrm{G}_{i_{0}}\right)=b_{1}\left(\mathrm{G}_{i_{0}+1}\right)=b_{1}\left(\mathrm{G}_{i_{0}+2}\right)=\ldots=b_{1}\left(\mathrm{~L}_{\infty}\right)
$$

so $b_{1}\left(\mathbf{L}_{\infty}\right) \geqslant 2$.

We will prove theorem 4.1 by induction on the first Betti number of a limit group. By lemma 4.2 a limit group $\mathrm{L}_{\infty}$ with $b_{1}\left(\mathrm{~L}_{\infty}\right)=1$ is cyclic, the analysis lattice of a cyclic group has only 1 level, so theorem 4.1 is valid for limit groups with first Betti 
number 1 . To be able to use the inductive hypothesis for proving the termination of the construction of the analysis lattice, we need the following fact.

Proposition 4.3. - Let $\mathrm{L}_{\infty}$ be a limit group. If $\mathrm{L}_{j}^{i}$ is a group in the $i$-th level of the analysis lattice of the limit group $\mathrm{L}_{\infty}, \mathrm{L}_{j^{\prime}}^{i+1}$ is connected to $\mathrm{L}_{j}^{i}$ and $\mathrm{L}_{j^{\prime \prime}}^{i+2}$ is connected to $\mathrm{L}_{j^{\prime}}^{i+1}$ in this lattice, then either $\mathrm{L}_{j^{\prime \prime}}^{i+2}$ is abelian or a surface group or a free group or:

(i) $b_{1}\left(\mathrm{~L}_{j^{\prime \prime}}^{i+2}\right)<b_{1}\left(\mathrm{~L}_{j}^{i}\right)$.

(ii) $\mathrm{L}_{j^{\prime}}^{i+1}$ is a subgroup of another limit group $\mathrm{D}_{j^{\prime}}^{i+1}$ for which: $b_{1}\left(\mathrm{D}_{j^{\prime}}^{i+1}\right)<b_{1}\left(\mathrm{~L}_{j}^{i}\right)$.

(iii) $\mathrm{L}_{j^{\prime \prime}}^{i+2}$ is a subgroup of another limit group $\mathrm{D}_{j^{\prime \prime}}^{i+2}$ for which: $b_{1}\left(\mathrm{D}_{j^{\prime \prime}}^{i+2}\right)<b_{1}\left(\mathrm{~L}_{j}^{i}\right)$.

(iv) $\mathrm{L}_{j^{\prime}}^{i+1}$ is a subgroup of another limit group $\mathrm{M}_{j^{\prime}}^{i+1}$ for which: $b_{1}\left(\mathrm{M}_{j^{\prime}}^{i+1}\right)=b_{1}\left(\mathrm{~L}_{j}^{i}\right)$, and the first Betti number of the graph corresponding to the canonical cyclic JSJ decomposition of $\mathrm{M}_{j}^{i+1}$ is strictly bigger than the first Betti number of the graph corresponding to the canonical cyclic JSJ decomposition of $\mathrm{L}_{j}^{i}$.

Proof. - We will assume that $\mathrm{L}_{j^{\prime \prime}}^{i+2}$ is not abelian, nor a surface group nor a free group. Since $\mathrm{L}_{j}^{i}$ is connected to groups in the $i+1$-level of the analysis lattice, $\mathrm{L}_{j}^{i}$, and similarly $\mathrm{L}_{j^{\prime}}^{i+1}$, are not abelian. If $\mathrm{M}=\mathrm{A} * \mathrm{~B}$ then $b_{1}(\mathbf{M})=b_{1}(\mathrm{~A})+b_{1}(\mathrm{~B})$. Since the groups connected to $\mathrm{L}_{j}^{i}$ in the $i+1$-level are vertex groups in the cyclic JSJ decomposition of freely indecomposable factors of $\mathrm{L}_{j}^{i}$, if $\mathrm{L}_{j^{\prime}}^{i+1}$ is connected to $\mathrm{L}_{j}^{i}$ in the analysis lattice then $b_{1}\left(\mathrm{~L}_{j^{\prime}}^{i+1}\right) \leqslant b_{1}\left(\mathrm{~L}_{j}^{i}\right)$. If $b_{1}\left(\mathrm{~L}_{j^{\prime}}^{i+1}\right)=b_{1}\left(\mathrm{~L}_{j}^{i}\right)$ then $\mathrm{L}_{j}^{i}$ must be freely indecomposable, and by lemma 4.2 and a simple homological computation only one vertex in its canonical cyclic JSJ decomposition is non-cyclic. By theorem 3.2 a limit group admits a principal cyclic splitting, so if $b_{1}\left(\mathrm{~L}_{j^{\prime}}^{i+1}\right)=b_{1}\left(\mathrm{~L}_{j}^{i}\right)$ the cyclic JSJ decomposition of $\mathrm{L}_{j}^{i}$ is not a tree of groups with one non-cyclic vertex group. Hence, if we suppose case (i) of the proposition does not hold, i.e., $b_{1}\left(\mathrm{~L}_{j}^{i}\right)=b_{1}\left(\mathrm{~L}_{j^{\prime}}^{i+1}\right)=b_{1}\left(\mathrm{~L}_{j^{\prime \prime}}^{i+2}\right)$, then the cyclic JSJ decompositions of $\mathrm{L}_{j}^{i}$ and $\mathrm{L}_{j^{\prime}}^{i+1}$ contain one vertex with non-cyclic vertex group, bouquet of circles based on this vertex and, perhaps, some additional vertices with cyclic stabilizers located either in the interior of a circle or connected by a unique edge to the vertex with non-trivial stabilizer.

To simplify notation we set $\mathrm{L}_{1}=\mathrm{L}_{j}^{i}, \mathrm{~L}_{2}=\mathrm{L}_{j^{\prime}}^{i+1}$ and $\mathrm{L}_{3}=\mathrm{L}_{j^{\prime \prime}}^{i+2}$. We set $\Lambda_{1}$ to be the cyclic JSJ decomposition of $\mathrm{L}_{1}$, and $\Lambda_{2}$ to be the cyclic JSJ decomposition of $\mathrm{L}_{2}$. The cyclic splitting $\Lambda_{1}$ has one vertex group, $L_{2}$, bouquet of circles, with cyclic edge groups $<c_{1}>, \ldots, \quad<c_{k}>$ and Bass-Serre generators $t_{1}, \ldots, t_{k}$ so that $t_{i} c_{i} t_{i}^{-1}=c_{i}^{\prime}$, and perhaps some additional vertices stabilized by cyclic vertex groups each connected by a single edge to the vertex stabilized by $\mathrm{L}_{2}$. We set $\widehat{\mathrm{L}_{2}}$ to be the subgroup of $\mathrm{L}_{1}$ generated by $\mathrm{L}_{2}$ and the cyclic vertex groups connected to the vertex stabilized by $\mathrm{L}_{2}$. The cyclic splitting $\Lambda_{2}$ has one vertex group, $\mathrm{L}_{3}$, and bouquet of circles, with cyclic edge groups $<e_{1}>, \ldots,<e_{\ell}>$ and Bass-Serre generators $b_{1}, \ldots, b_{\ell}$ so that $b_{j} e_{j} b_{j}^{-1}=e_{j}^{\prime}$, and perhaps 
some additional vertices stabilized by cyclic vertex groups each connected by a single edge to the vertex stabilized by $\mathrm{L}_{3}$. Let $e_{1}, \ldots, e_{q}$ be the entire collection of edge groups in $\Lambda_{2}$. We set $\widehat{\mathrm{L}_{3}}$ to be the subgroup of $\mathrm{L}_{2}$ generated by $\mathrm{L}_{3}$ and the cyclic vertex groups connected to the vertex stabilized by $\mathrm{L}_{3}$.

At this point we use the shortening process used in proving theorem 3.2, applied to the cyclic splitting $\Lambda_{1}$ of $\mathrm{L}_{1}$, and obtain a (new) limit group $\mathrm{D}_{\infty}$, together with a canonical map $v: \mathrm{L}_{1} \rightarrow \mathrm{D}_{\infty}$. By construction $v$ maps the group $\widehat{\mathrm{L}_{2}}$ monomorphically into $\mathrm{D}_{\infty}$. If $\mathrm{D}_{\infty}$ is freely decomposable, then either $\widehat{\mathrm{L}_{2}}$ is freely decomposable or $b_{1}\left(\mathrm{~L}_{2}\right) \leqslant b_{1}\left(\widehat{\mathrm{L}_{2}}\right)<b_{1}\left(\mathrm{D}_{\infty}\right) \leqslant b_{1}\left(\mathrm{~L}_{1}\right)$, so in both cases part (i) of the proposition holds. Hence, we may assume that $\mathrm{D}_{\infty}$ is freely indecomposable, and $\mathrm{D}_{\infty}$ is clearly not abelian, so $\mathrm{D}_{\infty}$ admits a canonical (non-trivial) cyclic JSJ decomposition. If $\mathrm{D}_{\infty}=\mathrm{U} *_{\mathrm{C}} \mathrm{M}$ where $\mathbf{C}$ is cyclic and $\mathrm{M}$ is non-cyclic abelian, then $\mathrm{L}_{3}<\mathrm{U}$ and $b_{1}(\mathbf{U}) \leqslant b_{1}\left(\mathrm{~L}_{\infty}\right)-1$, so if we set $\mathrm{D}_{j^{\prime \prime}}^{i+2}$ to be the subgroup $\mathrm{U}$ of $\mathrm{D}_{\infty}$, part (ii) of the proposition follows. Hence, we may assume that every non-cyclic abelian subgroup of $\mathrm{D}_{\infty}$ is elliptic in its canonical cyclic decomposition. We denote the cyclic JSJ decomposition of $\mathrm{D}_{\infty}, \Lambda_{\mathrm{D}}$. Note that by lemma 2.3, $\Lambda_{\mathrm{D}}$ is a 2-acylindrical splitting.

$\mathrm{D}_{\infty}$ is generated by the images (under the homomorphism $v$ ) of $\mathrm{L}_{2}$, the BassSerre generators $t_{1}, \ldots, t_{k}$ and the cyclic vertex groups in $\Lambda_{1}$. By the way the shortening argument was defined if $v\left(\mathrm{~L}_{2}\right)$ fixes a vertex in $\Lambda_{\mathrm{D}}$, or more generally, if all the elements $v\left(c_{1}\right), \ldots, v\left(c_{k}\right)$ fix the same vertex in $\Lambda_{\mathrm{D}}$, the cyclic splitting $\Lambda_{\mathrm{D}}$ is either degenerate or it is a tree of groups with only one non-cyclic vertex group, so $\mathrm{D}_{\infty}$ does not admit a principal cyclic splitting, a contradiction to theorem 3.2. Furthermore, since $\Lambda_{1}$ contains a unique non-cyclic vertex group, if all the elements $v\left(c_{1}\right), \ldots, v\left(c_{k}\right)$ are elliptic in $\Lambda_{\mathrm{D}}$, they must fix the same vertex in $\Lambda_{\mathrm{D}}$. Hence, $v\left(\mathrm{~L}_{2}\right)$ does not fix a vertex in $\Lambda_{\mathrm{D}}$, so it inherits a cyclic decomposition $\Gamma_{2}$ from $\Lambda_{\mathrm{D}}$, $\mathrm{L}_{3}$ fixes a vertex in $\Gamma_{2}$, this vertex is the only one stabilized by a non-cyclic group in $\Gamma_{2}$, and at least some of the elements $v\left(c_{i}\right)$ are not elliptic in $\Gamma_{2}$.

Let $\mathrm{V}_{\mathrm{D}}$ be the vertex group stabilized by $\mathrm{L}_{3}$ in $\Lambda_{\mathrm{D}}$. Since the cyclic JSJ decomposition of $\mathrm{L}_{2}$ contains a unique non-cyclic vertex group $\left(\mathrm{L}_{3}\right)$, if $\mathrm{v}\left(c_{i}\right)$ is elliptic in $\Lambda_{\mathrm{D}}$ for some $i, \mathrm{v}\left(c_{i}\right)$ fixes either the vertex stabilized by $\mathrm{V}_{\mathrm{D}}$ or a vertex connected to $\mathrm{V}_{\mathrm{D}}$ and stabilized by a cyclic vertex group in $\Gamma_{2}$.

Since $\boldsymbol{v}\left(c_{i}\right)=\mathbf{v}\left(t_{i}\right) \mathbf{v}\left(c_{i}^{\prime}\right) \mathbf{v}\left(t_{i}^{-1}\right)$, if $\boldsymbol{v}\left(c_{i}\right)$ is hyperbolic in $\Lambda_{\mathrm{D}}$ so is $\boldsymbol{v}\left(c_{i}^{\prime}\right)$. Since $\mathrm{L}_{2}$ is freely indecomposable, and the cyclic JSJ decomposition of $\mathrm{L}_{2}$ is $\Lambda_{2}$, each edge in the axis of $v\left(c_{i}\right)$ and $v\left(c_{i}^{\prime}\right)$ has to be stabilized by some conjugates (by elements in the subgroup $\left.v\left(\mathrm{~L}_{2}\right)\right)$ of the elements $v\left(e_{1}\right), \ldots, v\left(e_{q}\right) . v\left(t_{i}\right)$ maps the axis of $v\left(c_{i}\right)$ to the axis of $v\left(c_{i}^{\prime}\right)$.

First, suppose that $\boldsymbol{v}\left(t_{i}\right)$ maps an edge $\mathrm{E}$ stabilized by $v\left(a_{1} e_{j} a_{1}^{-1}\right)$ for some $a_{1} \in \mathrm{L}_{2}$ in the axis of $\mathrm{v}\left(c_{i}\right)$, to an edge $\mathrm{E}^{\prime}$ stabilized by $\mathrm{v}\left(a_{2} e_{j} a_{2}^{-1}\right)$ for some $a_{2} \in \mathrm{L}_{2}$. If we denote $a_{3}=a_{2} a_{1}^{-1}$ then the element $d_{i}=\mathbf{v}\left(t_{i}\right) \mathbf{v}\left(a_{3}^{-1}\right)$ fixes the edge E. Hence, there exists some 
element $e$ in the (cyclic) stabilizer of the edge E, for which $d_{i}=e$ and $v\left(t_{i}\right)=e v\left(a_{3}^{-1}\right)$. Since $e$ and $v\left(a_{1} e_{j} a_{1}^{-1}\right)$ belong to the same cyclic subgroup in $\mathrm{D}_{\infty}$, and $v\left(t_{i}\right)$ conjugates $\mathrm{v}\left(c_{i}\right)$ to $\mathrm{v}\left(c_{i}^{\prime}\right), b_{1}\left(<e, \mathrm{v}\left(\mathrm{L}_{2}\right)>\right) \leqslant b_{1}\left(\mathrm{~L}_{2}\right)-1<b_{1}\left(\mathrm{~L}_{1}\right)$. Hence, in this case we set $\mathrm{D}_{j^{\prime}}^{i+1}$ to be the subgroup $<e, v\left(\mathrm{~L}_{2}\right)>$ of $\mathrm{D}_{\infty}, b_{1}\left(\mathrm{D}_{j^{\prime}}^{i+1}\right)<b_{1}\left(\mathrm{~L}_{1}\right)$, and case (ii) holds.

Let $c_{1}, \ldots, c_{k^{\prime}}$ be the cyclic edge groups in $\Lambda_{1}$ for which $v\left(c_{1}\right), \ldots, v\left(c_{k^{\prime}}\right)$ are not elliptic in $\Lambda_{\mathrm{D}}$. Let $\mathrm{E}_{1}, \ldots, \mathrm{E}_{s}$ be the orbits of edges in $\Lambda_{\mathrm{D}}$ under the action of $\mathrm{L}_{2}$, that intersect non-trivially the axes of $v\left(c_{1}\right), \ldots, v\left(c_{k^{\prime}}\right), v\left(c_{1}^{\prime}\right), \ldots, v\left(c_{k^{\prime}}^{\prime}\right)$. Each of the (images of the) Bass-Serre generators $v\left(t_{i}\right)$ maps the axis of the element $v\left(c_{i}\right)$ to the axis of the element $\boldsymbol{v}\left(c_{i}^{\prime}\right)$, for $i=1, \ldots, k^{\prime}$, so each of the elements $\boldsymbol{v}\left(t_{i}\right)$ maps edges from the orbits $\mathrm{E}_{1}, \ldots, \mathrm{E}_{s}$ to edges from these orbits. We construct a (labeled) finite graph $\Delta$ with vertices corresponding to the orbits $\mathrm{E}_{1}, \ldots, \mathrm{E}_{s}$, and we connect two vertices $\mathrm{E}_{j_{1}}, \mathrm{E}_{j_{2}}$ by an edge, if an element $\mathbf{v}\left(t_{i}\right)$ maps an edge that is in the orbit of $\mathrm{E}_{j_{1}}$ to an edge that is in the orbit of $\mathrm{E}_{j_{2}}$. In this case we label the edge $\mathrm{E}_{j_{1}}, \mathrm{E}_{j_{2}}$ with the label $v\left(t_{i}\right)$. Note that for each label $\mathbf{v}\left(t_{i}\right)$, there is at least one edge in $\Delta$ that is labeled by $\mathbf{v}\left(t_{i}\right)$.

If there are loops in $\Delta$, then the argument given above implies that $\mathrm{L}_{2}$ can be embedded in a limit group $\mathrm{D}_{j^{\prime}}^{i+1}$, for which $b_{1}\left(\mathrm{D}_{j^{\prime}}^{i+1}\right)<b_{1}\left(\mathrm{~L}_{j}^{i}\right)$, and case (ii) of the proposition holds. Hence, we may assume that $\Delta$ contains no loops. If there is a circle in $\Delta$ for which the edges in this circle are labeled by distinct labels, then the same argument applies, so we may assume that there are no such circles. Therefore, the number of orbits $\mathrm{E}_{1}, \ldots, \mathrm{E}_{s}$ is strictly bigger than the number of labels $\mathrm{v}\left(t_{1}\right), \ldots, \mathrm{v}\left(t_{k^{\prime}}\right)$.

$\mathrm{L}_{2}$ inherits a graph of groups $\Gamma_{2}$ from $\Lambda_{\mathrm{D}}$, that has one non-abelian vertex group and a bouquet of $s$ circles, in which the elements $c_{k^{\prime}+1}, \ldots, c_{k}$ and $c_{k^{\prime}+1}^{\prime}, \ldots, c_{k}^{\prime}$ are elliptic. We set the limit group $\mathrm{M}$, that is a subgroup of $\mathrm{L}_{j}^{i}$, to be the fundamental group of the graph of groups obtained from $\Gamma_{2}$ by adding loops with edge groups $c_{k^{\prime}+1}, \ldots, c_{k}$ and Bass-Serre generators $t_{k^{\prime}+1}, \ldots, t_{k}$ that conjugate $c_{i}$ to $c_{i}^{\prime}$ in correspondence, for $i=k^{\prime}+1, \ldots, k$.

By construction, $b_{1}(\mathbf{M}) \leqslant b_{1}\left(\mathrm{~L}_{j}^{i}\right)$ and $\mathrm{L}_{2}$ is embedded in $\mathrm{M}$. If $b_{1}(\mathrm{M})<b_{1}\left(\mathrm{~L}_{j}^{i}\right)$ then case (ii) of the proposition holds, hence, we may assume that $b_{1}(\mathbf{M})=b_{1}\left(\mathbf{L}_{j}^{i}\right)$. If $\mathbf{M}$ is freely decomposable, then either $\mathrm{L}_{2}$ is freely indecomposable or $\mathrm{L}_{2}$ can be embedded into a limit group with a strictly smaller Betti number, so either case (i) or case (ii) of the proposition hold. Hence, we can assume that $\mathrm{M}$ is freely indecomposable. If the cyclic JSJ decomposition of $\mathrm{M}$ contains more than one non-cyclic vertex group, then $\mathrm{L}_{3}$ can be embedded into a limit group with a strictly smaller Betti number, and case (iii) of the proposition holds. Hence, we may assume that the cyclic JSJ decomposition of $\mathrm{M}$, that we denote $\Delta_{1}$, contains a unique non-cyclic vertex group, and at least $k+1$ loops based on the vertex that is stabilized by that non-cyclic vertex group. In this case, $b_{1}(\mathbf{M})=b_{1}\left(\mathrm{~L}_{j}^{i}\right), \mathrm{L}_{2}=\mathrm{L}_{j^{\prime}}^{i+1}$ is embedded into $\mathrm{M}$, and the Betti number of the graph associated with the cyclic JSJ decomposition of $\mathrm{M}, \Delta_{1}$, is strictly bigger than the first 
Betti number of the graph associated with the cyclic JSJ decomposition of $\mathrm{L}_{j}^{i}$, hence, case (iv) holds.

At this point we are ready to complete the proof of theorem 4.1 by induction on the first Betti number of $\mathrm{L}_{\infty}$. If $b_{1}\left(\mathrm{~L}_{\infty}\right)=1, \mathrm{~L}_{\infty}$ is cyclic by lemma 4.2 and its analysis lattice contains only one level. Suppose $b_{1}\left(\mathrm{~L}_{\infty}\right)>1$. Let $\mathrm{L}_{j^{\prime}}^{2}, \mathrm{~L}_{j^{\prime \prime}}^{3}$ be the beginning of a branch of the analysis lattice. If $\mathrm{L}_{j^{\prime}}^{2}$ or $\mathrm{L}_{j^{\prime \prime}}^{3}$ are abelian, free or surface groups, then they are terminal points in the analysis lattice, and the construction of the analysis lattice terminates along the given branch. If cases (i)-(iii) of proposition 4.3 hold, then $\mathrm{L}_{j^{\prime}}^{2}$ or $\mathrm{L}_{j^{\prime \prime}}^{3}$ can be embedded into a limit group with strictly smaller Betti number, so the construction of the analysis lattice along our given path terminates after finitely many steps by our inductive hypothesis. If case (iv) of proposition 4.3 holds, then $\mathrm{L}_{j^{\prime}}^{2}$ can be embedded in a limit group $\mathrm{M}_{j^{\prime}}^{2}$, with the same Betti number as that of $\mathrm{L}_{\infty}$, but for which the Betti number of the graph corresponding to the cyclic JSJ decomposition of $\mathrm{M}_{j}^{2}$ is strictly bigger than the Betti number of the graph corresponding to the cyclic JSJ decomposition of $\mathrm{L}_{\infty}$. Since the Betti number of the graph corresponding to a graph of groups is bounded by the Betti number of the fundamental group of the graph of groups, case (iv) of proposition 4.3 can be iteratively applied only finitely many times before one of the cases (i)-(iii) applies, and our inductive hypothesis can be applied to imply the termination of the construction of the analysis lattice after finitely many steps.

The termination of the construction of the analysis lattice is the key technical tool for understanding the algebraic structure of limit groups. In fact it can be viewed as a (canonical) "Haken Hierarchy" for limit groups. The following algebraic properties of limit groups are an immediate corollary. Similar properties of $\omega$-residually free groups (that are shown to be limit groups in the sequel) were proven by $\mathrm{O}$. Kharlampovich and A. Myasnikov (see corollaries 3 and 4 of theorem 6 in [Kh-My]).

Corollary 4.4. - Let $\mathrm{L}_{\infty}$ be a limit group. Then:

(i) $\mathrm{L}_{\infty}$ is finitely presented.

(ii) An abelian subgroup of $\mathrm{L}_{\infty}$ is a f.g. free abelian group. The rank of a free abelian subgroup is bounded by the first Betti number of $\mathrm{L}_{\infty}$.

(iii) If $\mathrm{L}_{\infty}$ does not contain a non-cyclic abelian subgroup then $\mathrm{L}_{\infty}$ is (Gromov) hyperbolic.

Proof. - The analysis lattice shows that a limit group can be constructed from abelian, free, and surface groups by a finite sequence of amalgamated products and HNN extensions over cyclic groups and free products, hence, it is finitely presented, and we get (i). Every abelian subgroup of a limit group which is not cyclic or $\mathrm{Z}+\mathrm{Z}$ is elliptic in its cyclic JSJ decomposition. Therefore, such abelian subgroup of $\mathrm{L}_{\infty}$ must 
appear as a terminal vertex group in the analysis lattice, so it must be f.g. and its rank is bounded by the first Betti number of $\mathrm{L}_{\infty}$. A limit group is torsion-free by lemma 1.3 which gives us part (ii). If $\mathrm{L}_{\infty}$ does not contain non-cyclic abelian groups, it is constructed from free and surface groups by a sequence of amalgamated products and HNN extensions along cyclic groups and free products, and since every cyclic subgroup of a limit group is contained in a unique maximal cyclic one by lemma 1.4 and part (ii), such limit group is a hyperbolic group by the combination theorem of M. Bestvina and M. Feighn [Be-Fe3].

The finite presentability of limit groups is a key tool in our analysis of sets of solutions to equations. It is the source for all the finiteness results we will get both for these sets and in dealing with the elementary theory of free groups in our continuation papers. An immediate implication of the finite presentability is equivalence between limit groups and f.g. $\omega$-residually free groups.

Definition 4.5. - Let $\mathrm{H}$ be a group. $\mathrm{H}$ is called residually free if for every element $h \neq 1$ in $\mathrm{H}$, there exists a free group $\mathrm{F}_{k}$ and a homomorphism $\phi: \mathrm{H} \rightarrow \mathrm{F}_{k}$ so that $\phi(h) \neq 1 . \mathrm{H}$ is called $\omega$-residually free if for any integer $m$ and every set of $m$ elements $h_{1}, \ldots, h_{m} \neq 1$ in $\mathrm{H}$, there exist an integer $k$ and a homomorphsim $\phi: \mathrm{H} \rightarrow \mathrm{F}_{k}$ so that $\phi\left(h_{1}\right), \ldots, \phi\left(h_{n}\right) \neq 1$.

Theorem 4.6. - A f.g. group $\mathrm{R}$ is a limit group if and only if it is $\omega$-residually free.

Proof. - The theorem is obvious for f.g. free abelian groups, so we may assume the f.g. group $\mathrm{R}$ is not abelian.

Suppose $\mathrm{R}$ is a limit group. By definition there exists a f.g. group $\mathrm{G}$, an integer $k$ and a sequence of homomorphisms $h_{n}: \mathrm{G} \rightarrow \mathrm{F}_{k}$, so that the limit of the actions of $\mathrm{G}$ on the Cayley graph of $\mathrm{F}_{k}$ via the homomorphisms $h_{n}$ is a faithful action of $\mathrm{R}$ on some real tree $\mathrm{Y}$.

By corollary $4.4 \mathrm{R}$ is f.p. so by lemma 1.3 for all but finitely indices $n$, the homomorphisms $h_{n}$ split through the limit group $\mathrm{R}$, i.e., $h_{n}=\psi_{n} \circ p$ where $p: \mathrm{G} \rightarrow \mathrm{R}$ is the canonical projection map, and the $\psi_{n}$ 's are homomorphisms $\psi_{n}: \mathrm{R} \rightarrow \mathrm{F}_{k}$. By lemma 1.3, if $r \neq 1$ in $\mathrm{R}$, then for all but finitely many $n$ 's $\psi_{n}(r) \neq 1$. Hence, for every integer $m$ and every set of $m$ elements $r_{1}, \ldots, r_{m} \neq 1$ in $\mathrm{R}$, for all but finitely many indices $n, \psi_{n}\left(r_{1}\right), \ldots, \psi_{n}\left(r_{m}\right) \neq 1$, so $\mathrm{R}$ is $\omega$-residually free.

To prove the other direction, suppose $\mathrm{R}=\left\langle r_{1}, \ldots, r_{s}\right\rangle$ is f.g. and $\omega$-residually free. Since $\mathrm{R}$ is $\omega$-residually free there exists a sequence of homomorphisms $\phi_{n}$ : $\mathrm{R} \rightarrow \mathrm{F}_{2}$, so that $\phi_{n}$ maps the elements in a ball of radius $m$ in the Cayley graph of $\mathrm{R}$ to distinct elements in $\mathrm{F}_{2}$. By rescaling the metric on $\mathrm{F}_{2}$, proposition 1.1 implies that there exists a subsequence of the homomorphisms $\phi_{n}$ which converges to an action of a limit group L on a real tree Y. In general, the limit group L is a quotient of the f.g. group $\mathrm{R}$, but since the homomorphisms were chosen so that $\phi_{n}$ maps a ball of radius $n$ 
"monomorphically" into $\mathrm{F}_{2}$, part (iii) of lemma 1.3 implies that $\mathrm{R}$ is isomorphic to $\mathrm{L}$ and, therefore, $\mathrm{R}$ is a limit group.

Clearly, since f.g. $\omega$-residually free groups are limit groups and vice versa, all the algebraic properties proven for limit groups are valid for $\omega$-residually free groups. In particular, they admit a principal cyclic decomposition (theorem 3.2), a canonical cyclic JSJ decomposition (theorem 3.9), a (canonical) analysis lattice, they are f.p., every abelian subgroup of a f.g. $\omega$-residually free group is a f.g. free abelian group and its rank is bounded by the first Betti number of the ambient $\omega$-residually free group, and if a f.g. $\omega$-residually free group does not contain a non-cyclic abelian subgroup it is (Gromov) hyperbolic. Also, since a f.g. $\omega$-residually free group is, in particular, residually finite, it satisfies the Hopf property.

\section{The (Ganonical) Makanin-Razborov Diagram}

The algebraic properties of limit groups proved in the previous sections introduce tools that initialize the analysis of these groups, but are still short of obtaining any kind of classification of them. i.e., the cyclic JSJ decomposition and the analysis lattice associated with a limit group, are helpful in proving properties of limit groups but they are not enough for getting a necessary and sufficient conditions for a f.p. group to be a limit group (or equivalently an $\omega$-residually free group). To get such criteria, we need to introduce canonical resolutions of limit groups, resolutions which will capture all the possible homomorphisms from a limit group into a free group and which are similar in nature to the ones appear in works of Makanin [Ma] and Razborov [Ral], obtained there under some additional combinatorial hypothesis (bounded periodicity), and generalized later by Razborov in his dissertation [Ra2], and by Kharlampovich and Myasnikov in [Kh-My].

On the set of limit groups ( $\omega$-residually free groups) we define a partial order. Given two limit groups $R_{1}, R_{2}$ we say that $R_{1}>R_{2}$ if $R_{2}$ is a proper quotient of $R_{1}$. Note that since a limit group is Hopf, if $R_{2}$ is a proper quotient of $R_{1}$ then $R_{1}$ can not be a quotient of $\mathbf{R}_{2}$. A first implication of the finite presentability of limit groups to their partial order is the termination of decreasing sequences.

Proposition 5.1. - Every sequence of decreasing limit groups $\mathrm{R}_{1}>\mathrm{R}_{2}>\mathrm{R}_{3}>\ldots$ terminates.

Proof. - Suppose there is a decreasing sequence which does not terminate. The limit groups $\left\{\mathrm{R}_{m}\right\}$ are all quotients of $\mathrm{R}_{1}$, so we may assume their fixed finite set of generators is the image of a fixed finite set of generators of $R_{1}$. The limit groups $R_{m}$ are $\omega$-residually free by theorem 4.6, so for each fixed $m$ we can define a sequence of homomorphisms $h_{n}^{m}: \mathrm{R}_{m} \rightarrow \mathrm{F}_{k}$ so that $h_{n}^{m}$ maps the $n$-ball in the Cayley graph of $\mathbf{R}_{m}$ to 
distinct elements in $\mathrm{F}_{k}$. From the sequences $\left\{h_{n}^{m}\right\}$ we extract the diagonal sequence of homomorphisms $\phi_{m}: \mathrm{R}_{1} \rightarrow \mathrm{F}_{k}$ by setting $\phi_{m}=h_{m}^{m} \circ p_{m}$ where $p_{m}: \mathrm{R}_{1} \rightarrow \mathrm{R}_{m}$ is the natural projection. By rescaling the metric on $\mathrm{F}_{k}$, proposition 1.1 implies that the actions of $\mathbf{R}_{1}$ on the Cayley graph of $\mathrm{F}_{k}$ via the homomorphisms $\left\{\phi_{m}\right\}$ subconverge into an action of a limit group $\mathrm{R}$ on a real tree $\mathrm{Y}$ (see section 1 ).

$\mathrm{R}$ is a quotient of all the groups $\mathrm{R}_{m}$ in the decreasing sequence and it is a limit group by construction. By corollary $4.4 \mathrm{R}$ is f.p. and by part (iii) of lemma 1.3 for all but finitely many indices $m$, the (finite) defining relations of $\mathrm{R}$ are mapped to the trivial element by the homomorphisms $\phi_{m}$. Since the homomorphisms $h_{m}^{m}$ were chosen to map the ball of radius $m$ in the Cayley graph of $\mathbf{R}_{m}$ into distinct elements, and since the defining relations of $\mathrm{R}$ are mapped to the identity by $\phi_{m}$, for all but finitely many $m$ 's, there exists some index $m_{0}$, so that $\mathbf{R}_{m_{0}}, \mathbf{R}_{m_{0}+1}, \ldots$ are all quotients of the limit group $\mathrm{R}$. But $\mathrm{R}$ is a proper quotient of the $\mathrm{R}_{m}$ 's, so we obtain a contradiction to the Hopf property of the limit group $\mathrm{R}$, and a decreasing sequence must terminate.

To get an understanding of the structure of limit groups ( $\omega$-residually free groups) we need to find a way to "encode" all possible homomorphisms from a limit group into free groups. To get such "encoding" we will construct a canonical diagram associated with a limit group. To construct this canonical diagram we need to introduce shortening quotients of a limit group. To construct a shortening quotient of a limit group we need to define its associated modular group.

Definition 5.2. — Let $\mathrm{R}$ be a freely-indecomposable limit group. We define the modular group $\operatorname{Mod}(\mathrm{R})$ to be the subgroup of $A u t(\mathrm{R})$ generated by the following families of automorphisms of $\mathrm{R}$ :

(i) Inner automorphisms.

(ii) Dehn twists along edges of the cyclic JSJ decomposition of $\mathrm{R}$.

(iii) Dehn twists along essential s.c.c. in CMQ (canonical maximal quadratically hanging) vertex groups in the cyclic JSJ decomposition of $\mathrm{R}$.

(iv) Let $\mathrm{A}$ be an abelian vertex group in the cyclic JSJ decomposition of $\mathrm{R}$, and let $\mathrm{A}_{1}<\mathrm{A}$ be the subgroup generated by all the edge groups connecting $\mathrm{A}$ to the other vertex groups in the cyclic JSJ decomposition of $\mathrm{R}$. Every automorphism of $\mathrm{A}$ that fixes $\mathrm{A}_{1}$ (elementwise) can be naturally extended to an automorphism of the ambient limit group $\mathrm{R}$. We call these generalized Dehn twists and they form the fourth family of automorphisms that generate $\operatorname{Mod}(\mathbf{R})$.

At this stage we are ready to modify the shortening argument, presented in ([Be], 7.4) and in section in order to obtain the shortening quotients of a freely-indecomposable limit group $\mathrm{R}$. Let $\left\{h_{n} \mid h_{n}: \mathrm{R} \rightarrow \mathrm{F}_{k}\right\}$ be a sequence of homomorphisms from $\mathrm{R}$ into a free group $\mathrm{F}_{k}$. Let $r_{1}, \ldots, r_{m} \in \mathrm{R}$ be a generating set of $\mathrm{R}$, and let $\mathrm{V}_{1}, \ldots, \mathrm{V}_{\ell}$ be the vertex groups in the cyclic JSJ decomposition of $\mathrm{R}$. 
Let $\mathrm{X}$ be the Cayley graph of the free group $\mathrm{F}_{k}$ with respect to a (fixed) finite set of generators, and let $d_{\mathrm{X}}$ be the standard simplicial metric on $\mathrm{X}$. For each element $f \in \mathrm{F}_{k}$, each positive integer $n$, and each automorphism $\varphi \in \operatorname{Mod}(\mathrm{R})$ we define the following (stretching) constant:

$$
\mu(n, f, \varphi)=\max \left(d_{\mathrm{X}}\left(i d ., f h_{n}\left(\varphi\left(r_{1}\right)\right) f^{-1}\right), \ldots, d_{\mathrm{X}}\left(i d ., f h_{n}\left(\varphi\left(r_{m}\right)\right) f^{-1}\right)\right) .
$$

For each $n$ we choose $f_{n} \in \mathrm{F}_{k}$ and $\varphi_{n} \in \operatorname{Mod}(\mathbf{R})$ for which $\mu\left(n, f_{n}, \varphi_{n}\right)$ is minimal in the set $\{\mu(n, f, \varphi)\}$. We set $\operatorname{dis}_{n}=\mu\left(n, f_{n}, \varphi_{n}\right)$.

The limit group $\mathrm{R}$ admits a natural action on the Cayley graph $\mathrm{X}$ of $\mathrm{F}_{k}$ which we denote $\rho_{n}: \mathrm{R} \times \mathrm{X} \rightarrow \mathrm{X}$, by setting $\rho_{n}(r, x)=\tau_{f_{n}} \circ h_{n} \circ \varphi_{n}(r)(x)$ for every $r \in \mathrm{R}$ and $x \in \mathrm{X}$, where $\tau_{f_{n}}$ is the inner automorphism of $\mathrm{F}_{k}$ defined by $f_{n}$. Since $\mathrm{X}$ is a simplicial tree, for the $n$-th action $\rho_{n}$ we may rescale the metric on the Cayley graph $\mathrm{X}$ by $\operatorname{dis}_{n}$, and apply proposition $1.1([\mathrm{~Pa}], 2.3)$ for the sequence of actions $\left\{\rho_{n}\right\}$ to obtain a subsequence (still denoted $\left\{\boldsymbol{\rho}_{n}\right\}$ ) converging into a (pointed) real tree $\left(\mathrm{T}, t_{0}\right)$ equipped with a non-trivial isometric action of $\mathrm{R}$. We set $\mathrm{KS}_{\infty}$ to be the kernel of the action of $\mathrm{R}$ on the real tree $\mathrm{T}$, and the limit group $\mathrm{S}_{\infty}$ to be the quotient $\mathrm{S}_{\infty}=\mathrm{R} / \mathrm{K} \mathrm{S}_{\infty}$. We say that the limit group $S_{\infty}$ associated with the canonical quotient map $\eta: R \rightarrow S_{\infty}$ is a shortening quotient of the limit group $\mathrm{R}$. Note that the construction of a shortening quotient of $\mathrm{R}$ does depend on the sequence of homomorphisms $h_{n}: \mathrm{R} \rightarrow \mathrm{F}_{k}$ and the specific generating set $r_{1}, \ldots, r_{m} \in \mathrm{R}$ pre-chosen for $\mathrm{R}$.

We say that two shortening quotients $\mathrm{S}_{1}, \mathrm{~S}_{2}$ of the limit group $\mathrm{R}$ are equivalent, if there exists an isomorphism $\tau: S_{1} \rightarrow S_{2}$, and the canonical map $\eta_{2}: R \rightarrow S_{2}$ can be expressed as $\eta_{2}=\tau \circ \eta_{1} \circ \varphi$ where $\varphi \in \operatorname{Mod}(\mathrm{R})$ and $\eta_{1}: \mathrm{R} \rightarrow \mathrm{S}_{1}$ is the canonical map associated with the (maximal) shortening quotient $\mathrm{S}_{1}$. The notion of equivalent shortening quotients is clearly an equivalence relation on the set of couples of shortening quotients and their associated canonical maps: $\left\{\left(\mathrm{S}_{i}, \eta_{i}: \mathrm{R} \rightarrow \mathrm{S}_{i}\right)\right\}$ of the limit group R.

Since all the homomorphisms $h_{n}$ map the limit group $\mathrm{R}$ into a free group $\mathrm{F}_{k}$, a shortening quotient of a limit group $\mathrm{R}$ is indeed a quotient of $\mathrm{R}$. In fact, it is a proper quotient of it.

Claim 5.3. — Let $\mathrm{R}$ be a freely-indecomposable limit group. Then every shortening quotient of $\mathrm{R}$ is a proper quotient of $\mathrm{R}$.

Proof. — Let R be a freely-indecomposable limit group, let $\left\{h_{n}: \mathrm{R} \rightarrow \mathrm{F}_{k}\right\}$ be a sequence of homomorphisms, suppose the sequence of actions of $\mathrm{R}$ on the Cayley graph of $\mathrm{F}_{k}$ via the homomorphisms $\left\{h_{n}\right\}$ converge into an action of a shortening quotient $\mathrm{S}$ of $\mathrm{R}$ on some real tree $\mathrm{Y}_{\mathrm{S}}$, and suppose each of the homomorphisms $h_{n}$ is the "shortest" possible under compositions with modular automorphisms of $\mathrm{R}$ and inner automorphisms of $\mathrm{F}_{k}$. 
Now, assume that the shortening quotient $\mathrm{S}$ is isomorphic to $\mathrm{R}$, so the canonical map $\eta: R \rightarrow S$ is an isomorphism. Let $\Gamma_{R}$ be the abelian JSJ decomposition of $\mathrm{R}$ (see theorem 2.7). By theorem 3.2 R obtains a principal cyclic splitting, so some of the edge groups in $\Gamma_{\mathrm{R}}$ are cyclic.

To obtain a contradiction to the isomorphism between $\mathrm{R}$ and its shortening quotient S, suppose first that all the edge groups in the abelian JSJ decomposition of $\mathrm{R}$ are cyclic. By theorem 1.5, the shortening quotient $\mathrm{S}$ inherits an abelian splitting from its action on the limit tree $\mathrm{Y}_{\mathrm{S}}$. Since $\mathrm{R}$ is assumed isomorphic to its shortening quotient $\mathrm{S}, \mathrm{R}$ inherits an abelian splitting from its action on $\mathrm{Y}_{\mathrm{S}}$.

The limit action of $\mathrm{S}$ on $\mathrm{Y}_{\mathrm{S}}$ was obtained from a sequence of homomorphisms $\left\{h_{n}: \mathrm{R} \rightarrow \mathrm{F}_{k}\right\}$ that were assume the "shortest" possible under compositions with modular automorphisms of $\mathrm{R}$ and inner automorphisms of $\mathrm{F}_{k}$. Therefore, the real tree $\mathrm{Y}_{\mathrm{S}}$ can not contain an IET component, since otherwise, for large enough $n$, it was possible to "shorten" the homomorphism $h_{n}$ by pre-composing with a modular automorphism of $\mathrm{R}$ by the canonical properties of the abelian JSJ decomposition of $\mathrm{R}$ (theorem 2.7) and the shortening argument given in ([Ri-Sel], 5.1), which contradicts our assumptions on the homomorphisms $\left\{h_{n}\right\}$. By a similar argument $\mathrm{Y}_{\mathrm{S}}$ does not contain an axial component. Hence, the action of $\mathrm{R}$ on $\mathrm{Y}_{\mathrm{S}}$ is discrete. But again, when the action of $\mathrm{R}$ on $\mathrm{Y}_{\mathrm{S}}$ is discrete, for large enough $n$, it is possible to "shorten" the homomorphisms $h_{n}$ by precomposing with a modular automorphism of $\mathrm{R}$ by the canonical properties of the abelian JSJ decomposition of $\mathrm{R}$ (theorem 2.7) and the shortening argument given in ([Se3], 2.5), a contradiction to our assumptions on the homomorphisms $\left\{h_{n}\right\}$. Hence, the abelian JSJ decomposition of $\mathrm{R}, \Gamma_{\mathrm{R}}$, must contain some abelian, non-cyclic, edge groups.

Let $\mathrm{E}_{1}, \ldots, \mathrm{E}_{\ell}$ be the abelian non-cyclic edge groups in $\Gamma_{\mathrm{R}}$, let $\Lambda_{1}, \ldots, \Lambda_{q}$ be the subgraphs of $\Gamma_{\mathrm{R}}$ which are the connected components of the graph obtained from $\Gamma_{\mathrm{R}}$ by deleting the edges stabilized by $\mathrm{E}_{1}, \ldots, \mathrm{E}_{\ell}$. Since $\mathrm{R}$ admits a principal cyclic splitting by theorem 3.2, the abelian JSJ decomposition $\Gamma_{R}$ contains an edge with cyclic edge group which corresponds to a principal cyclic splitting of $\mathrm{R}$, and this edge must be contained in one of the connected subgraphs $\Lambda_{1}, \ldots, \Lambda_{q}$, without loss of generality, $\Lambda_{1}$.

Let $\mathrm{R}_{1}$ be the subgroup of $\mathrm{R}$ which is the fundamental group of the subgraph $\Lambda_{1}$. Let $\left\{h_{n}^{1}: \mathrm{R}_{1} \rightarrow \mathrm{F}_{k}\right\}$ be the restrictions of the homomorphisms $\left\{h_{n}: \mathrm{R} \rightarrow \mathrm{F}_{k}\right\}$ to the subgroup $\mathrm{R}_{1}$. From the sequence of actions of $\mathrm{R}_{1}$ on the Cayley graph of $\mathrm{F}_{k}$ via the homomorphisms $\left\{h_{n}^{1}\right\}$ it is possible to extract a subsequence (still denoted $\left\{h_{n}^{1}\right\}$ ) that converge into an action of $\mathrm{R}_{1}$ on some real tree $\mathrm{Y}_{1}$. By theorem $1.5 \mathrm{R}_{1}$ inherits a (non-trivial) abelian splitting $\Gamma_{1}$ from its action on $\mathrm{Y}_{1}$.

Let $\mathrm{E}_{1}, \ldots, \mathrm{E}_{u}$ be the abelian non-cyclic edge groups in $\Gamma_{\mathrm{R}}$ which are the stabilizers of edges connected to $\Lambda_{1}$, i.e., $\mathrm{E}_{1}, \ldots, \mathrm{E}_{u}$ are the edge groups in $\Gamma_{\mathrm{R}}$ which are also subgroups of $\mathrm{R}_{1}$. If any of the groups $\mathrm{E}_{1}, \ldots, \mathrm{E}_{u}$ is not elliptic when acting on the limit tree $Y_{1}$, it is possible to further refine the abelian JSJ decomposition $\Gamma_{R}$ of $R$, 
which contradicts its canonical properties (theorem 2.7). Hence, the subgroups $\mathrm{E}_{1}, \ldots, \mathrm{E}_{u}$ can all be conjugated into vertex groups in $\Gamma_{1}$, so $\Gamma_{1}$ can be extended to an abelian splitting on the ambient group $\mathrm{R}$, so by the canonical properties of the abelian JSJ decomposition of $\mathrm{R}, \Gamma_{1}$ can be obtained from the connected subgraph $\Lambda_{1}$ of the abelian JSJ decomposition of $\mathrm{R}, \Gamma_{\mathrm{R}}$.

Now, modifying the "shortening argument" in case all edge groups in $\Gamma_{\mathrm{R}}$ are cyclic, if the real tree $\mathrm{R}_{1}$ contains an IET component, for large enough $n$, it is possible to "shorten" the homomorphisms $h_{n}^{1}$ by pre-composing with a modular automorphism of $\mathrm{R}$ that preserve $\mathrm{R}_{1}$ by the canonical properties of the abelian JSJ decomposition of $\mathrm{R}$ (theorem 2.7) and the shortening argument given in ([Ri-Sel], 5.1). By construction a composition of these shortening modular automorphisms with the ambient homomorphisms $\left\{h_{n}: \mathrm{R} \rightarrow \mathrm{F}_{k}\right\}$ make these "shorter", which contradicts our assumptions on the homomorphisms $\left\{h_{n}\right\}$. By a similar argument $\mathrm{Y}_{1}$ does not contain an axial component. Hence, the action of $\mathrm{R}_{1}$ on $\mathrm{Y}_{1}$ is discrete. But again, when the action of $\mathrm{R}_{1}$ on $\mathrm{Y}_{1}$ is discrete, for large enough $n$, it is possible to "shorten" the homomorphisms $h_{n}^{1}$ by precomposing with a modular automorphism of $\mathrm{R}$ by the canonical properties of the abelian JSJ decomposition of $\mathrm{R}$ (theorem 2.7) and the shortening argument given in ([Se3], 2.5). By construction a composition of these shortening modular automorphisms with the ambient homomorphisms $\left\{h_{n}: \mathrm{R} \rightarrow \mathrm{F}_{k}\right\}$ make these "shorter", a contradiction to our assumptions on the homomorphisms $\left\{h_{n}\right\}$.

Let $\mathrm{SQ}\left(\mathrm{R}, r_{1}, \ldots, r_{m}\right)$ be the set of shortening quotients of $\mathrm{R}$. On the set $\mathrm{SQ}\left(\mathrm{R}, r_{1}, \ldots, r_{m}\right)$ we define a partial order as follows. For given elements $\mathrm{S}_{1}, \mathrm{~S}_{2} \in$ $\mathrm{SQ}\left(\mathrm{R}, r_{1}, \ldots, r_{m}\right)$ we say that $\mathrm{S}_{1}>\mathrm{S}_{2}$ if $\mathrm{S}_{2}$ is a proper quotient of $\mathrm{S}_{1}$ and the canonical map $\eta_{2}: R \rightarrow S_{2}$ splits as $\eta_{2}=v \circ \eta_{1}$ where $\eta_{1}: R \rightarrow S_{1}$ is the canonical map associated with $\mathrm{S}_{1}$ and $\mathrm{v}: \mathrm{S}_{1} \rightarrow \mathrm{S}_{2}$ is a homomorphism.

Lemma 5.4. - Let $\mathrm{R}$ be a freely-indecomposable limit group. Let $\mathrm{S}_{1}<\mathrm{S}_{2}<\mathrm{S}_{3}<\ldots$ (where $\mathrm{S}_{j} \in \mathrm{SQ}\left(\mathrm{R}, r_{1}, \ldots, r_{m}\right)$ ) be a properly increasing sequence of shortening quotients of $\mathrm{R}$. Then there exists a shortening quotient $\mathrm{S} \in \mathrm{SQ}\left(\mathrm{R}, r_{1}, \ldots, r_{m}\right)$ so that $\mathrm{S}>\mathrm{S}_{j}$ for every shortening quotient $\mathrm{S}_{j}$ in the increasing sequence.

Proof. - Let $\mathrm{S}_{1}<\mathrm{S}_{2}<\mathrm{S}_{3}<\ldots$ be an infinite properly increasing sequence of shortening quotients of the freely-indecomposable limit group R. Since each of the groups $\mathrm{S}_{j}$ is a shortening quotient of $\mathrm{R}$, for each $j$ there exists a sequence of homomorphisms $h_{n}^{j}: \mathrm{R} \rightarrow \mathrm{F}_{k}$, so that $h_{n}^{j}$ can not be shortened by compositions with both a modular automorphism of $\mathrm{R}$ and an inner automorphism of $\mathrm{F}_{k}$, and if $r \in \mathrm{R}$ for which $\eta_{j}(r)$ is not the identity element in $\mathrm{S}_{j}$ and $\eta_{j}(r)$ is contained in the ball of Radius $n$ in the Cayley graph of $\mathrm{S}_{j}$, then $h_{n}^{j}(r) \neq 1$. 
Now, we look at the diagonal sequence $h_{j}^{j}: \mathrm{R} \rightarrow \mathrm{F}_{k}$. By the construction used in section 1 , we may choose a converging subsequence (to simplify notation still denoted $h_{j}^{j}$ ), so that the sequence of homomorphisms $h_{j}^{j}: \mathrm{R} \rightarrow \mathrm{F}_{k}$ converges into an action of a limit group $\mathrm{S}$ on some real tree $\mathrm{Y}$. By our assumptions on the homomorphisms $h_{n}^{j}: \mathrm{R} \rightarrow \mathrm{F}_{k}$, the diagonal sequence $h_{j}^{j}: \mathrm{R} \rightarrow \mathrm{F}_{k}$ can not be shortened by compositions with both a modular automorphism of $\mathrm{R}$ and an inner automorphism of $\mathrm{F}_{k}$, so the limit group $\mathrm{S}$ is a shortening quotient of $\mathrm{R}$, hence, $\mathrm{S} \in \mathrm{SQ}\left(\mathrm{R}, r_{1}, \ldots, r_{m}\right)$.

The shortening quotient $\mathrm{S}$ is in particular a limit group, so by corollary 4.4 it is f.p. By lemma 1.3 there must exist some index $j_{0}$ so that for all indices $j>j_{0}$, the homomorphisms $h_{j}^{j}$ map the (finite set of) defining relations of $\mathrm{S}$ to the trivial element in $\mathrm{F}_{k}$. Since by the way the homomorphisms $h_{n}^{j}$ were chosen, $h_{j}^{j}$ maps a ball of radius $j / 2$ in $\mathrm{S}_{j}$ monomorphically into $\mathrm{F}_{k}$, all the defining relations of $\mathrm{S}$ must be trivial in $\mathrm{S}_{j}$ for large enough $j$. Therefore, $\mathrm{S}>\mathrm{S}_{j}$ for large enough $j$ which clearly implies that $\mathrm{S}>\mathrm{S}_{j}$ for all indices $j$.

Lemma 5.4 proves the existence of maximal elements with respect to the partial order on the set of shortening quotients $\mathrm{SQ}\left(\mathrm{R}, r_{1}, \ldots, r_{m}\right)$. The next lemma shows that there are only finitely many equivalence classes of maximal elements in the set of shortening quotients.

Lemma 5.5. - Let $\mathrm{R}$ be a freely-indecomposable limit group. The set of shortening quotients of $\mathrm{R}, \mathrm{SQ}\left(\mathrm{R}, r_{1}, \ldots, r_{m}\right)$, contains only finitely many equivalence classes of maximal elements with respect to its partial order.

Proof. - Suppose there are infinitely many non-equivalent maximal shortening quotients $\mathrm{S}_{1}, \mathrm{~S}_{2}, \mathrm{~S}_{3}, \ldots$, of some freely-indecomposable limit group $\mathrm{R}$. As we did in the proof of lemma 5.4 since each of the groups $\mathrm{S}_{j}$ is a shortening quotient of $\mathrm{R}$, for each $j$ there exists a sequence of homomorphisms $h_{n}^{j}: \mathrm{R} \rightarrow \mathrm{F}_{k}$, so that $h_{n}^{j}$ can not be shortened by compositions with both a modular automorphism of $\mathrm{R}$ and an inner automorphism of $\mathrm{F}_{k}$, and if $r \in \mathrm{R}$ for which $\eta_{j}(r)$ is not the identity element in $\mathrm{S}_{j}$ and $\eta_{j}(r)$ is contained in the ball of Radius $n$ in the Cayley graph of $\mathrm{S}_{j}$, then $h_{n}^{j}(r) \neq 1$.

From the diagonal sequence $h_{j}^{j}: \mathrm{R} \rightarrow \mathrm{F}_{k}$ we can extract a convergent subsequence (still denote $h_{j}^{j}$ ), so that the sequence of homomorphisms $h_{j}^{j}: \mathrm{R} \rightarrow \mathrm{F}_{k}$ converges into an action of a limit group $\mathrm{S}$ on some real tree $\mathrm{Y}$, and by our assumptions on the homomorphisms $h_{n}^{j}: \mathrm{R} \rightarrow \mathrm{F}_{k}$ the limit group $\mathrm{S}$ is a shortening quotient of $\mathrm{R}$, i.e., $\mathrm{S} \in \mathrm{SQ}\left(\mathrm{R}, r_{1}, \ldots, r_{m}\right)$.

The shortening quotient $\mathrm{S}$ is in particular a limit group, so by corollary 4.4 it is f.p. By lemma 1.3 there must exist some index $j_{0}$ so that for all indices $j>j_{0}$, the homomorphisms $h_{j}^{j}$ map the (finite set of) defining relations of $\mathrm{S}$ to the trivial element in $\mathrm{F}_{k}$. Since by the way the homomorphisms $h_{n}^{j}$ were chosen, $h_{j}^{j}$ maps a ball of radius 
$j / 2$ in $\mathrm{S}_{j}$ monomorphically into $\mathrm{F}_{k}$, all the defining relations of $\mathrm{S}$ must be trivial in $\mathrm{S}_{j}$ for some $j>j_{1}$. Therefore, for every $j>j_{1}$ either $\mathrm{S}>\mathrm{S}_{j}$ or $\mathrm{S}$ is equivalent to $\mathrm{S}_{j}$. But all the shortening quotients $\mathrm{S}_{j}$ are assumed maximal in $\mathrm{SQ}\left(\mathrm{R}, r_{1}, \ldots, r_{m}\right)$, so for all $j>j_{1} \mathrm{~S}$ is equivalent to $\mathrm{S}_{j}$, and that contradicts our assumption on the $\mathrm{S}_{j}$ 's being from different equivalence classes of shortening quotients of $\mathrm{R}$.

The main significance of maximal shortening quotients is the way they "encode" and simplify all homomorphisms from a freely-indecomposable limit group into a free group.

Proposition 5.6. - Let $\mathrm{R}$ be a freely-indecomposable limit group. Let $r_{1}, \ldots, r_{m} \in \mathrm{R}$ be a generating set of $\mathrm{R}$, let $\mathrm{M}_{1}, \ldots, \mathrm{M}_{k}$ be a collection of representatives of the (finite) set of equivalence classes of maximal shortening quotients in $\mathrm{SQ}\left(\mathrm{R}, r_{1}, \ldots, r_{m}\right)$, and for $i=1, \ldots, k$ let $\eta_{i}: \mathrm{R} \rightarrow \mathbf{M}_{i}$ be the canonical projection maps.

Let $h: \mathrm{R} \rightarrow \mathrm{F}_{k}$ be a homomorphism. Then there exists some index $1 \leqslant i \leqslant k$ (not necessarily unique!) and a modular automorphism $\varphi_{h} \in \operatorname{Mod}(\mathrm{R})$ so that $h \circ \varphi_{h}$ splits through the maximal shortening quotient $\mathrm{M}_{i}$, i.e., $h \circ \varphi_{h}=h_{\mathrm{M}_{i}} \circ \eta_{i}$ where $h_{\mathrm{M}_{i}}: \mathrm{M}_{i} \rightarrow \mathrm{F}_{k}$ is a homomorphism.

Proof. - Given a homomorphism $h: \mathrm{R} \rightarrow \mathrm{F}_{k}$ we can choose a modular automorphism $\varphi_{h} \in \operatorname{Mod}(\mathrm{R})$ and an inner automorphism $\tau_{f} \in \operatorname{Inn}\left(\mathrm{F}_{k}\right)$ so that the composition $\widehat{h}=\tau_{f} \circ h \circ \varphi_{h}$ is the shortest possible among all such compositions. The constant sequence of homomorphisms $\widehat{h}, \widehat{h}, \ldots$ converges into a free limit group $\mathrm{F}$ which is a subgroup of $\mathrm{F}_{k}$. By our assumption on $\widehat{h}, \mathrm{~F}$ is a shortening quotient and the map $\widehat{h}: \mathrm{R} \rightarrow \mathrm{F}$ is its associated canonical map. By lemmas 5.4 and 5.5, either there exists some maximal element $\mathrm{M}_{i}>\mathrm{F}$, or one of the $\mathrm{M}_{i}$ 's is equivalent to $\mathrm{F}$. In both cases the homomorphism $\widehat{h}: \mathrm{R} \rightarrow \mathrm{F}$ splits through the maximal shortening quotient $\mathrm{M}_{i}$ and the proposition follows.

The shortening procedure and the lemmas and propositions proved so far in this section finally allow us to present the main goal of this section, the (canonical) Makanin-Razborov diagram associated with a limit group ( $\omega$-residually free group). The Makanin-Razborov diagram "encodes" all possible homomorphisms from a limit group into a free group, and as we will later show, some of its properties can be stated as a criteria for a general f.g. group to be a limit group.

Let $\mathrm{R}$ be a limit group and let $\mathrm{R}=\mathrm{R}_{1} * \ldots \mathrm{R}_{\ell} * \mathrm{~F}_{g_{1}}$ be a factorization of $\mathrm{R}$, where each of the $\mathbf{R}_{i}$ 's is a freely-indecomposable non-cyclic subgroup of $\mathbf{R}$ and $\mathbf{F}_{g_{1}}$ is a free group. Let $r_{1}^{1}, \ldots, r_{m_{1}}^{1} \in \mathrm{R}$ be a generating set of $\mathrm{R}_{1}, r_{1}^{2}, \ldots, r_{m_{2}}^{2}$ be a generating set of $\mathrm{R}_{2}$, etc. By lemma 5.4 the sets of shortening quotients of the freely-indecomposable limit groups $\mathrm{R}_{1}, \ldots, \mathrm{R}_{\ell}$ contain maximal elements (with respect to the partial order defined above) and by lemma 5.5 there are only finitely many equivalence classes of maximal 
shortening quotients of each of the limit groups $\mathbf{R}_{1}, \ldots, \mathbf{R}_{\ell}$. For $i=1, \ldots, \ell$ let $\mathbf{M}_{1}^{i}, \ldots, \mathbf{M}_{k_{i}}^{i}$ be a collection of representatives of equivalence classes of maximal shortening quotients in $\mathrm{SQ}\left(\mathrm{R}_{i}, r_{1}^{i}, \ldots, r_{m_{i}}^{i}\right)$, and let $\eta_{j}^{i}: \mathrm{R}_{i} \rightarrow \mathrm{M}_{j}^{i}$ be the canonical projection maps.

We define the Makanin-Razborov diagram of the limit group $\mathrm{R}$ iteratively. We start by factoring $\mathrm{R}$ into its freely-indecomposable factors $\mathrm{R}_{1}, \ldots, \mathrm{R}_{\ell}$ and the free factor $\mathrm{F}_{g}$. From each of the factors $\mathrm{R}_{i}$ we associate $k_{i}$ directed edges, starting at $\mathbf{R}_{i}$ and terminating at the maximal shortening quotient $\mathbf{M}_{j}^{i}$. To each such directed edge we associate the canonical projection $\eta_{j}^{i}$. Note that we do not proceed from the free factor $\mathrm{F}_{g_{1}}$.

We proceed iteratively. We factor each of the $\mathbf{M}_{j}^{i}$ s into freely-indecomposable factors and associate with each such factor representatives for its equivalence classes of maximal shortening quotients. Since each (maximal) shortening quotient of a limit group is a proper quotient of that limit group by claim 5.3, and each sequence of properly decreasing sequence of limit groups terminates by proposition 5.1, the construction of the Makanin-Razborov diagram terminates.

By proposition 5.6 all the homomorphisms from a limit group $\mathrm{R}$ into a free group $\mathrm{F}_{k}$ are encoded by the canonical Makanin-Razborov diagram.

Theorem 5.7. — Let $\mathrm{R}$ be a limit group. All the homomorphisms $h: \mathrm{R} \rightarrow \mathrm{F}_{k}$ are given by compositions of modular automorphisms of the limit groups in the diagram with the canonical maps from the limit groups into their maximal shortening quotients and finally with general homomorphisms ("substitutions") of the terminal free groups that appear in the diagram into the target free group.

By Grushko's theorem the factorization of a f.g. group into freely-indecomposable factors is canonical. Once we fix a generating set of the limit group $\mathrm{R}$, the (equivalence classes of) the maximal shortening quotients constructed along the various levels of the Makanin-Razborov diagram are canonical as well, hence, the entire Makanin-Razborov diagram associated with a limit group $\mathrm{R}$ and a fixed generated set of $\mathrm{R}$, is canonical.

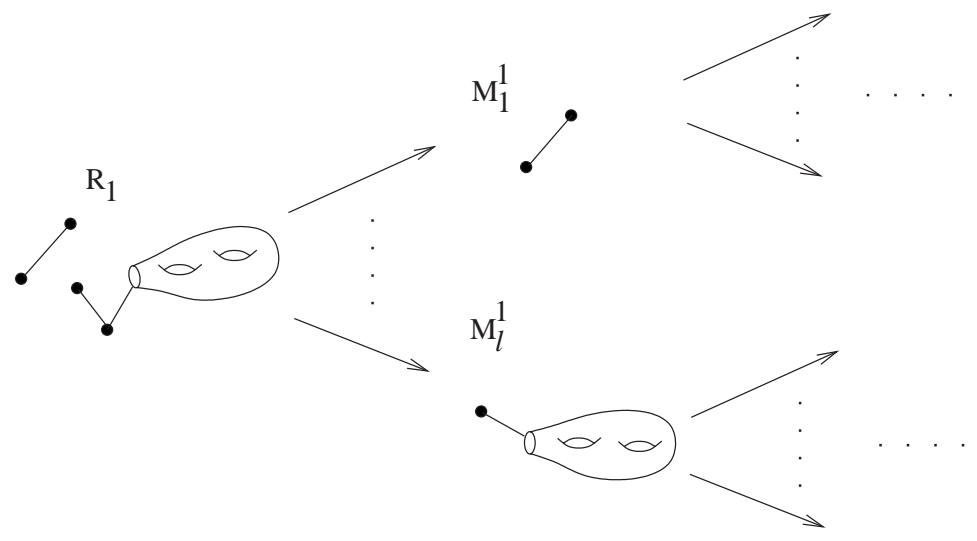


The (canonical) Makanin-Razborov diagram associated with a limit group encodes all the homomorphisms from that limit group into free groups. Some specific subdiagrams of it can be viewed as a criteria for a general f.g. group to be a limit group ( $\omega$-residually free group).

Definition 5.8. - A subdiagram of the Makanin-Razborov diagram in which for each free factor $\mathbf{R}_{i}$ of the limit group $\mathbf{R}$ we choose a unique edge connecting $\mathbf{R}_{i}$ to one of its maximal shortening quotients, and proceed iteratively choosing only one maximal shortening quotient at each stage, is called a Makanin-Razborov resolution.

To present a criteria for a f.g. group to be a limit group we need to show the existence of special type of Makanin-Razborov resolutions which we call strict MakaninRazborov resolutions.

Definition 5.9. - Let $\mathrm{R}$ be a freely-indecomposable limit group. We say that a shortening quotient $\mathrm{S}$ of $\mathrm{R}$ is a strict shortening quotient if:

(i) The subgroups generated by each non-CMQ non-abelian vertex group together with centralizers of edge groups connected to it in the graph of groups obtained from the cyclic JSJ of $\mathrm{R}$ by replacing each abelian vertex group by the direct summand containing the edge groups connected to it, and the cyclic edge groups in the cyclic JSJ decomposition of $\mathrm{R}$ are mapped monomorphically into the shortening quotient $\mathrm{S}$ by the canonical map $\eta: \mathrm{R} \rightarrow \mathrm{S}$..

(ii) Each CMQ subgroup of $\mathrm{R}$ is mapped to a non-abelian subgroup of $\mathrm{S}$ by the canonical map $\eta$, and each boundary element of a $\mathrm{CMQ}$ subgroup of $\mathrm{R}$ is mapped to a non-trivial element in $\mathrm{S}$ by $\eta$.

(iii) Let $\mathrm{A}$ be an abelian vertex group in the JSJ decomposition of $\mathrm{R}$, and let $\mathrm{A}_{1}<\mathrm{A}$ be the subgroup generated by all edge groups connected to the vertex stabilized by $\mathrm{A}$. Then $\mathrm{A}_{1}$ is mapped monomorphically into $\mathrm{S}$ by the canonical map $\eta$.

A Makanin-Razborov resolution is called strict if all the maximal shortening quotients appear in it are strict shortening quotients.

Finally, the existence of a strict Makanin-Razborov resolution is a criteria for a f.g. group to be a limit group ( $\omega$-residually free group). We first show that a limit group admits a strict Makanin-Razborov resolution.

Proposition 5.10. - Let $\mathrm{R}$ be a limit group. Then the (canonical) Makanin-Razborov diagram of $\mathrm{R}$ contains a strict Makanin-Razborov resolution.

Proof. - Let $\mathrm{R}$ be a freely-indecomposable limit group. $\mathrm{R}$ is an $\omega$-residually free group, so there exists a sequence of homomorphisms $h_{n}: \mathrm{R} \rightarrow \mathrm{F}_{k}$ so that $h_{n}$ maps the 
ball of radius $n$ in $\mathrm{R}$ monomorphically into $\mathrm{F}_{k}$ and the sequence of actions of $\mathrm{R}$ on the Cayley graph of $\mathrm{F}_{k}$ via the sequence of homomorphisms $\left\{h_{n}\right\}$ converges into a faithful action of $\mathrm{R}$ on some real tree $\mathrm{Y}$.

By composing each of the elements $h_{n}$ with an appropriate modular automorphism $\varphi_{n} \in \operatorname{Mod}(\mathrm{R})$ and an inner automorphism $\tau_{f_{n}} \in \operatorname{Inn}\left(\mathrm{F}_{k}\right)$ we obtain homomorphisms $\widehat{h}_{n}: \mathrm{R} \rightarrow \mathrm{F}_{k}, \widehat{h}_{n}=\tau_{f_{n}} \circ h_{n} \circ \varphi_{n}$, which are the shortest with respect to all such compositions. From the sequence $\widehat{h}_{n}$ we can extract a subsequence (still denoted $\widehat{h}_{n}$ ) that converge into an action of a shortening quotient $\mathrm{S}$ on some real tree T. Let $\eta: \mathrm{R} \rightarrow \mathrm{S}$ be the canonical map from $\mathrm{R}$ onto its shortening quotient $\mathrm{S}$.

If $r \in \mathrm{R}$ is contained in a non-CMQ non-abelian vertex group or a in a cyclic edge group in the cyclic JSJ decomposition of R, then $\varphi(r)$ is conjugate to $r$ for all $\varphi \in \operatorname{Mod}(\mathrm{R})$, hence, for all indices $n>n_{r}: h_{n}(r) \neq 1$ in $\mathrm{F}_{k}$, so by lemma $1.3 \eta(r) \neq 1$ in the shortening quotient $\mathrm{S}$ and every non-GMQ non-abelian vertex group and every cyclic edge group in the cyclic JSJ decomposition of $\mathrm{R}$ is mapped monomorphically into $\mathrm{S}$ by the canonical map $\eta$.

Similarly, if $\mathrm{A}$ is an abelian vertex group in the cyclic JSJ decomposition of $\mathrm{R}$ and $\mathrm{A}_{1}<\mathrm{A}$ is the subgroup generated by all edges connected to the vertex stabilized by $\mathrm{A}$ in the cyclic JSJ decomposition, then every modular automorphism $\varphi \in \operatorname{Mod}(\mathrm{R}) \operatorname{maps}$ the subgroup $A_{1}$ into a conjugate, hence, the canonical map $\eta$ maps $A_{1}$ monomorphically into the shortening quotient $\mathrm{S}$.

Let $\mathrm{V}$ be a GMQ-vertex group in the cyclic JSJ decomposition of R. Since the homomorphism $h_{n}$ is supposed to map the ball of radius $n$ in $\mathrm{R}$ monomorphically into $\mathrm{F}_{k}$, and since $\mathrm{V}$ is non-abelian in $\mathrm{R}$, there must exist some index $n_{0}$, so that for all indices $n>n_{0}, h_{n}(\mathrm{~V})$ is a non-abelian subgroup of $\mathrm{F}_{k}$. Since for every modular automorphism $\varphi \in \operatorname{Mod}(\mathrm{R}), \varphi(\mathrm{V})$ is conjugate to $\mathrm{V}, \widehat{h}_{n}(\mathrm{~V})$ is non-abelian subgroup of $\mathrm{F}_{k}$ for all $n>n_{0}$ as well. By lemma 1.3 this implies that $\eta(\mathrm{V})$ is a non-abelian subgroup of $\mathrm{F}_{k}$. A modular automorphism of $\mathrm{R}$ map the edge groups in the canonical JSJ decomposition of $\mathrm{R}$ into their conjugates, hence, the edge groups in the cyclic JSJ decomposition of $\mathrm{R}$ are mapped to non-trivial subgroups of $\mathrm{S}$ by the canonical map $\eta$.

So far we have shown that the shortening quotient $\mathrm{S}$ is a strict shortening quotient of $\mathrm{R}$. If $\mathrm{S}$ is not a maximal shortening quotient of $\mathrm{R}$, then there must exist a maximal shortening quotient $\mathrm{M}$ of $\mathrm{R}$ so that $\mathrm{M}>\mathrm{S}$. Clearly, since $\mathrm{S}$ is a strict shortening quotient of $\mathrm{R}$, the maximal shortening quotient $\mathrm{M}>\mathrm{S}$ must be strict as well.

Therefore, we have shown that for every freely-indecomposable limit group there exists a strict maximal shortening quotient. Now, by the way the Makanin-Razborov diagram of a limit group is constructed together with the existence of a strict maximal shortening quotient for every freely-indecomposable limit group, it follows that every Makanin-Razborov diagram contains a strict Makanin-Razborov resolution. 
Proposition 5.10 shows that a limit group admits a strict Makanin-Razborov resolution. To state a criteria for a f.g. group to be a limit group we need to define an analogue of a strict Makanin-Razborov resolution in the general context of f.g. groups.

Definition 5.11. - Let $\mathrm{G}$ be a f.g. group, and let:

$$
\mathrm{G}=\mathrm{G}_{0} \stackrel{v_{0}}{\longrightarrow} \mathrm{G}_{1} \stackrel{\mathrm{v}_{1}}{\longrightarrow} \mathrm{G}_{2} \stackrel{\mathrm{v}_{2}}{\longrightarrow} \ldots \stackrel{v_{m-2}}{\longrightarrow} \mathrm{G}_{m-1} \stackrel{v_{m-1}}{\longrightarrow} \mathrm{G}_{m}=\mathrm{F}_{u}
$$

be a resolution of $\mathrm{G}$. We say that the given resolution of $\mathrm{G}$ is a strict $\mathrm{MR}$ resolution if the epimorphisms $\mathrm{v}_{i}$ have the following properties.

First, we start with a (possible) free factorization of $\mathrm{G}, \mathrm{G}=\mathrm{G}^{1} * \ldots * \mathrm{G}^{n} * \mathrm{~F}^{s}$, where $\mathrm{F}^{s}$ is a free subgroup of $\mathrm{G}$. Each of the factors $\mathrm{G}^{j}$ of $\mathrm{G}=\mathrm{G}_{0}$ is mapped by the epimorphism $\mathrm{v}_{0}: \mathrm{G} \rightarrow \mathrm{G}_{1}$ onto the factor $\mathrm{Q}^{j}$ in a free factorization $\mathrm{G}_{1}=\mathrm{Q}^{1} * \ldots * \mathrm{Q}^{n}$ of $\mathrm{G}_{1}$. We further assume that each $\mathrm{G}^{j}$ admits a non-trivial cyclic splitting $\Lambda_{\mathrm{G}^{j}}$ with the following properties:

(i) Each cyclic edge group in $\Lambda_{\mathrm{G}^{j}}$ is a maximal abelian subgroup in at least one of the vertex groups it is connected to.

(ii) $v_{0}$ maps each of the subgroups generated by a non-QH (quadratically hanging), nonabelian vertex groups and the centralizers of the edges connected to it in the graph of groups obtained from the given cyclic splitting by replacing each abelian vertex group with the direct summand containing the edge groups connected to it, and each of the cyclic edge groups in $\Lambda_{\mathrm{G}}{ }^{j}$ monomorphically into $\mathrm{Q}^{j}$.

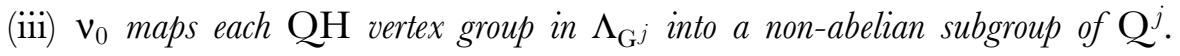

(iv) Every abelian vertex group in $\Lambda_{\mathrm{G}^{j}}$ is non-cyclic free abelian, and if $\mathrm{A}$ is an abelian vertex group in $\Lambda_{\mathrm{G}^{j}}$, and $\mathrm{A}_{1}<\mathrm{A}$ is the subgroup generated by all edge groups connected to the vertex stabilized by $\mathrm{A}$ in $\Lambda_{\mathrm{G}} j$, then $\mathrm{A}_{1}$ is mapped monomorphically into $\mathrm{Q}^{j}$ by the map $\boldsymbol{v}_{0}$.

Finally, we assume that the epimorphisms $\boldsymbol{v}_{i}$ associated with the next levels of the resolution of $\mathrm{G}$ satisfy similar conditions to the ones listed for $\mathrm{v}_{0}$, and the resolution terminates when the target groups are free (like in the construction of a Makanin-Razborov resolution of a limit group).

Note that a strict Makanin-Razborov resolution of a limit group $\mathrm{R}$ is a strict $\mathrm{MR}$ resolution of $\mathrm{R}$, so by proposition 5.11 a limit group admits a strict MR resolution. Theorem 5.12 shows that this is also a sufficient condition for a f.g. group to be a limit group.

Theorem 5.12. - A f.g. group $\mathrm{G}$ is a limit group ( $\omega$-residually free group) if and only if it admits a strict $\mathrm{MR}$ resolution

Proof. - Let:

$$
\mathrm{G}=\mathrm{G}_{0} \stackrel{v_{0}}{\longrightarrow} \mathrm{G}_{1} \stackrel{v_{1}}{\longrightarrow} \mathrm{G}_{2} \stackrel{v_{2}}{\longrightarrow} \ldots \stackrel{v_{m-2}}{\longrightarrow} \mathrm{G}_{m-1} \stackrel{v_{m-1}}{\longrightarrow} \mathrm{G}_{m}=\mathrm{F}_{u}
$$


be a strict MR-resolution of a f.g. group $G$, and let $\left\{\Lambda_{\mathrm{G}_{i}}\right\}$ be the cyclic splittings associated with each of the subgroups $\mathrm{G}_{i}$. We need to show the existence of sequences of modular automorphsims $\left\{\varphi_{i}(n) \in \operatorname{Mod}\left(\mathrm{G}_{i}\right)\right\}$ for $i=0, \ldots, m-1$ so that the limit group corresponding to the sequence of homomorphisms:

$$
\left\{h_{n}: \mathrm{G} \rightarrow \mathrm{F}_{k} \mid h_{n}=\mathrm{v}_{m-1} \varphi_{m-1}(n) \boldsymbol{v}_{m-2} \varphi_{m-2}(n) \ldots \boldsymbol{v}_{1} \varphi_{1}(n) \boldsymbol{v}_{0} \varphi_{0}(n)\right.
$$

is the group $\mathrm{G}$ itself.

If none of the cyclic splittings $\Lambda_{\mathrm{G}_{i}}$ contains a $\mathrm{QH}$ vertex groups, we may pick the automorphisms $\left\{\varphi_{i}(n)\right\}$ to be an increasing sequence of Dehn twists corresponding to the edges in the cyclic splittings $\Lambda_{\mathrm{G}_{i}}$. In the presence of $\mathrm{QH}$ vertex groups we need the following technical lemma.

Lemma 5.13. - Let $\mathrm{Q}$ be the fundamental group of a (possibly punctured) surface $\mathrm{S}_{\mathrm{Q}}$ of Euler characteristic at most -2 . Let $\mu: \mathrm{Q} \rightarrow \mathrm{F}_{k}$ be a homomorphism and suppose $\mathrm{Q}$ is mapped into a non-abelian subgroup of $\mathrm{F}_{k}$ and the image of every boundary component of $\mathrm{Q}$ is non-trivial. Then either:

(i) There exists a separating s.c.c. $\gamma \subset \mathrm{S}_{\mathrm{Q}}$ such that $\gamma$ is mapped non-trivially into $\mathrm{F}_{k}$, and the image in $\mathrm{F}_{k}$ of the fundamental groups of each of the connected components obtained by cutting $\mathrm{S}_{\mathrm{Q}}$ along $\gamma$ is non-abelian.

(ii) There exists a non-separating s.c.c. $\gamma \subset \mathrm{S}_{\mathrm{Q}}$ such that $\gamma$ is mapped non-trivially into $\mathrm{F}_{k}$, and the image of the fundamental group of the connected component obtained by cutting $\mathrm{S}_{\mathrm{Q}}$ along $\gamma$ is non-abelian.

Proof. - First, assume $\mathrm{S}_{\mathrm{Q}}$ is orientable. If $\mathrm{S}_{\mathrm{Q}}$ is a closed surface or a surface with a single puncture then clearly either (i) or (ii) holds. If $\mathrm{S}_{\mathrm{Q}}$ has more than one boundary component and $\mathrm{S}_{\mathrm{Q}}$ is not a planar surface, then $\mathrm{S}_{\mathrm{Q}}$ is the connected sum of two orientable surfaces $S_{1}$ and $S_{2}$, so that $S_{1}$ has only one boundary component and $\mathrm{S}_{2}$ is a planar surface. If the fundamental group of $\mathrm{S}_{1}$ is mapped into a non-abelian subgroup of $\mathrm{F}_{k}$, then either (i) or (ii) hold as in the case of a surface with at most one puncture. If the fundamental group of $\mathrm{S}_{1}$ is mapped into a cyclic subgroup of $\mathrm{F}_{k}$, then the cyclic subgroup corresponding to the boundary of $S_{1}$ is necessarily mapped into the trivial subgroup in $\mathrm{F}_{k}$. If the fundamental group of $\mathrm{S}_{1}$ is mapped into a non-trivial cyclic subgroup of $\mathrm{F}_{k}$, then there must exists a non-separating s.c.c. in $\mathrm{S}_{1}$ that is mapped non-trivially into $\mathrm{F}_{k}$, so case (ii) holds. If the fundamental group of $\mathrm{S}_{1}$ is mapped trivially into $\mathrm{F}_{k}$, then there exists a non-boundary-parallel non-separating s.c.c. in $\mathrm{S}_{\mathrm{Q}}$ that is mapped non-trivially into $\mathrm{F}_{k}$, and again case (ii) holds.

If $\mathrm{S}_{\mathrm{Q}}$ is a planar surface, we order its boundary components $b_{1}, \ldots, b_{n}$. Note that by our assumptions each boundary component $b_{i}$ is mapped non-trivially into $\mathrm{F}_{k}$, and not all the images of the $b_{i}$ 's in $\mathrm{F}_{k}$ commute. It is not hard to see that there must exist a couple of consecutive boundary components $b_{i}, b_{i+1}$ so that the images of $b_{i}$ and $b_{i+1}$ in 


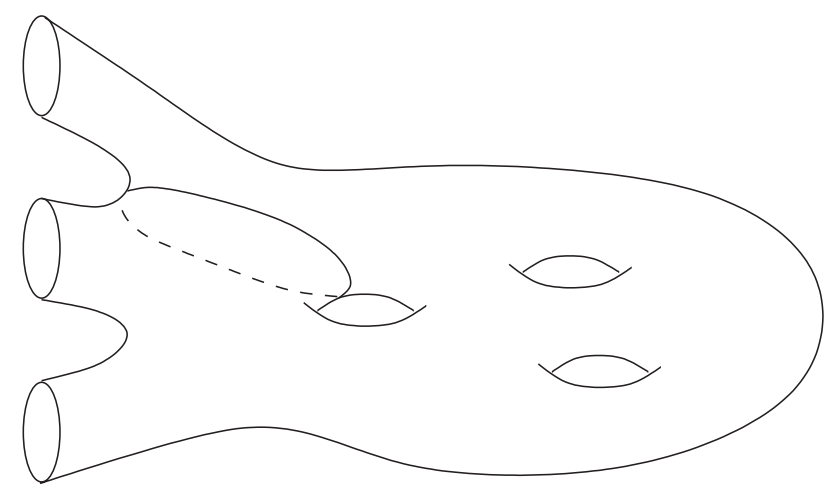

$\mathrm{F}_{k}$ do not commute, and the images of the elements $b_{1}, \ldots, b_{i-1}, b_{i} b_{i+1}, b_{i+2}, \ldots, b_{n}$ generate a nonabelian subgroup in $\mathrm{F}_{k}$. Hence, the separating s.c.c. in $\mathrm{S}_{\mathrm{Q}}$ corresponding to the element $b_{i} b_{i+1}$ is mapped to a non-trivial element in $\mathrm{F}_{k}$, and the fundamental groups of each of the connected components obtained by cutting $\mathrm{S}_{\mathbf{Q}}$ along this separating s.c.c. are mapped onto non-abelian subgroups of $\mathrm{F}_{k}$.

Now assume $\mathrm{S}_{\mathrm{Q}}$ is non-orientable. We can view $\mathrm{S}_{\mathrm{Q}}$ as the connected sum of an orientable surface $\mathrm{O}$ (with boundary) and a Mobius band $\mathrm{M}$. If the fundamental group of $\mathrm{M}$ is mapped non-trivially into $\mathrm{F}_{k}$, all the boundary components of $\mathrm{O}$ are mapped non-trivially into $\mathrm{F}_{k}$, and the fundamental group of $\mathrm{O}$ is mapped onto a non-abelian subgroup of $\mathrm{F}_{k}$, so our arguments above apply for the orientable surface $\mathrm{O}$, and there exists a s.c.c. $\gamma$ with the required properties.

If $\mathrm{S}_{Q}$ is a closed non-orientable surface, then $\mathrm{O}$ is an orientable surface with only one boundary component, and the fundamental group of $\mathrm{O}$ is mapped to a nonabelian subgroup of $\mathrm{F}_{k}$, hence, there exists a non-separating s.c.c. $\gamma \subset \mathrm{O}$ so that $\gamma$ is mapped non-trivially into $\mathrm{F}_{k}$ and the fundamental group of the connected component obtained by cutting $\mathrm{S}_{Q}$ along $\gamma$ is mapped to a non-trivial subgroup of $\mathrm{F}_{k}$.

If $\mathrm{S}_{\mathrm{Q}}$ has boundary, and the fundamental group of $\mathrm{M}$ is mapped to the identity in $\mathrm{F}_{k}$, then there exists a non-separating s.c.c. $\gamma \subset \mathrm{S}_{\mathrm{Q}}$ that cuts the Mobius band $\mathrm{M}$, so that $\gamma$ is mapped non-trivially into $\mathrm{F}_{k}$ and the fundamental group of the connected component obtained by cutting $\mathrm{S}_{\mathrm{Q}}$ along $\gamma$ is mapped to a non-abelian subgroup of $\mathrm{F}_{k}$.

By recursively applying lemma 5.13, for each surface $\mathrm{S}_{\mathrm{Q}_{j}}$ corresponding to a QH-vertex group in the cyclic splitting $\Lambda_{\mathrm{G}_{i}}$, we can find a finite set of s.c.c. on $\mathrm{S}_{\mathrm{Q}_{j}}$, so that each connected component of the surface obtained by cutting $\mathrm{S}_{\mathrm{Q}_{j}}$ along this family of s.c.c. has Euler characteristic -1, and the fundamental group of each of these connected components is mapped monomorphically into $\mathrm{G}_{i+1}$ under the map $v_{i}$.

If we extend each of the cyclic splittings $\Lambda_{\mathrm{G}_{i}}$ by further splitting the QH-vertex groups along the families of s.c.c. chosen according to lemma 5.13, the argument used 
to prove theorem 5.12 in the absence of $\mathrm{QH}$-subgroups generalizes to a give a proof in their presence.

\section{An Example: $\omega$-Residually Free Towers}

In the first 5 sections of this paper we studied the structure of $\omega$-residually free groups, using their JSJ decomposition, the analysis lattice and the Makanin-Razborov diagram and resolutions. Before applying the results and techniques used in studying $\omega$-residually free groups to study residually free groups and sets of solutions to equations in a free group which is the goal of this paper, we devote this section to some natural families of examples of $\omega$-residually free groups.

If $\mathrm{R}_{1}$ and $\mathrm{R}_{2}$ are $\omega$-residually free groups then $\mathrm{R}_{1} * \mathrm{R}_{2}$ is clearly an $\omega$-residually free group as well. If $\mathrm{R}$ is an $\omega$-residually free group, and $r \in \mathrm{R}$ is an element with cyclic centralizer and no non-trivial roots in $\mathrm{R}$, then the "double of $\mathrm{R}$ along $<r>$ ", $\mathrm{D}_{\mathrm{R}}=\mathrm{R} *_{<r} \mathrm{R}$, admits an obvious strict MR resolution, so by theorem 5.12 it is $\omega$ residually free. In particular, since a free and surface groups are $\omega$-residually free, every double of a free or a surface group along a maximal cyclic subgroup is an $\omega$-residually free group. Another more "geometric" family of $\omega$-residually free groups, which in some way is the motivation for most of the objects we have associated with a general $\omega$-residually free group, is the family of $\omega$-residually free towers. Note that an $\omega$ residually free tower is similar to the notion of a coordinate group of a non-degenerate quasiquadratic system introduced in [Kh-My].

Definition 6.1. - We say that a f.p. group $\mathrm{G}$ is an $\omega$-residually free tower, if it is possible to find a finite set set of generators $\mathrm{G}=<\left\{y_{j}^{\ell}\right\}>$ and defining relations $\left\{\boldsymbol{\varphi}_{b}^{\ell}\right\}$ for $\mathrm{G}$, where $1 \leqslant \ell \leqslant \ell(\mathrm{G}), 1 \leqslant j \leqslant m_{\ell}$ and $1 \leqslant b \leqslant r_{\ell}$, so that $\mathrm{G}$ can be presented using the following hierarchical structure which we define inductively:

(i) $\mathrm{G}^{0}$ is the trivial subgroup of $\mathrm{G}$.

(ii) With each level $\ell$ of the hierarchy we associate a group $\mathrm{G}^{\ell} . \mathrm{G}^{\ell}$ is the subgroup of $\mathrm{G}$ generated by the elements $\mathrm{G}^{\ell}=<\left\{y_{j}^{i}\right\}>$ where $1 \leqslant i \leqslant \ell$ and $1 \leqslant j \leqslant m_{i}$ in $\mathrm{G}$. $\mathrm{G}^{\ell}$ is the quotient of the free group generated by the elements $\left\{y_{j}^{i}\right\}$ for $1 \leqslant i \leqslant \ell$ and $1 \leqslant j \leqslant m_{i}$ divided by the normal closure of the set of relations $\varphi_{b}^{i}$ where $1 \leqslant i \leqslant \ell$ and $1 \leqslant b \leqslant r_{i}$.

(iii) To define the $\ell$-th level, the generators $y_{1}^{\ell}, \ldots, y_{m_{\ell}}^{\ell}$ are divided into $s^{\ell}$ disjoint blocks $y_{1}^{\ell}, \ldots, y_{b_{1}^{\ell}}^{\ell}, y_{b_{1}^{\ell}+1}^{\ell}, \ldots, y_{b_{2}^{\ell}}^{\ell}, \ldots, y_{b_{s}^{\ell} \ell_{-1}+1}^{\ell}, \ldots, y_{m_{\ell}}^{\ell}$ where to the $p$-th block of $y_{j}^{\ell}$ 's one of the following sets of defining relations is associated:

(1) a free block. No relation is associated to a free block of generators.

(2) an Abelian block. The relations $\left[\gamma_{j_{1}}^{\ell}, y_{j_{2}}^{\ell}\right]=1$ for every $b_{p-1}^{\ell}+1 \leqslant j_{1}<j_{2} \leqslant b_{p}^{i}$, and in addition the relation $\left[\bar{r}, y_{j}^{\ell}\right]=1$ for every $b_{p-1}^{\ell}+1 \leqslant j \leqslant b_{p}^{\ell}$ and some 
element $\bar{r} \in \mathrm{G}^{\ell-1}$ where $\bar{r}$ may be the identity element, if $\bar{r}$ is non-trivial it can not be conjugated into an Abelian block in lower levels, and $\bar{r}$ is not a proper power of an element in $\mathrm{G}^{\ell-1}$.

(3) a quadratic block. A single relation which is in one of the following two forms is being associated with a quadratic block. To save notation we denote the set of generators in the $p$-th block of $y_{j}^{\ell}$ which is a quadratic block by $y_{1}, \ldots, y_{2 g}, z_{1}, \ldots, z_{n}$ in the first case and by $y_{1}, \ldots, y_{g}, z_{1}, \ldots, z_{n}$ in the second. With this notation the relation associated with a quadratic block is either (orientable):

$$
\begin{gathered}
{\left[y_{1}, y_{2}\right] \ldots\left[y_{2 g-1}, y_{2 g}\right] z_{1} \bar{r}_{1} z_{1}^{-1} \ldots z_{n} \bar{r}_{n} z_{n}^{-1}=} \\
{\left[\bar{y}_{1}, \bar{y}_{2}\right] \ldots\left[\bar{y}_{2 g-1}, \bar{y}_{2 g}\right] \bar{z}_{1} \bar{r}_{1} \bar{z}_{1}^{-1} \ldots \bar{z}_{n} \bar{r}_{n} \bar{z}_{n}^{-1}}
\end{gathered}
$$

or (non-orientable):

$$
\begin{gathered}
y_{1}^{2} \ldots y_{2 g}^{2} z_{1} \bar{r}_{1} z_{1}^{-1} \ldots z_{n} \bar{r}_{n} z_{n}^{-1}= \\
\bar{y}_{1}^{2} \ldots \bar{y}_{2 g} \bar{z}_{1} \bar{r}_{1} \bar{z}_{1}^{-1} \ldots \bar{z}_{n} \bar{r}_{n} \bar{z}_{n}^{-1}
\end{gathered}
$$

where $\bar{r}_{j}, \bar{y}_{j}$ and $\bar{z}_{j}$ are elements in the group $\mathrm{G}^{\ell-1}$, the subgroups $<\left\{\bar{r}_{j}\right\},\left\{\bar{y}_{j}\right\}>$ and $<\left\{\bar{r}_{j}\right\},\left\{\bar{z}_{j}\right\}>$ are non-abelian subgroups of $\mathrm{G}^{\ell-1}$, the element $r_{i}$ is conjugate to the element $\bar{r}_{i}$ in $\mathrm{G}^{\ell}$, and the (punctured) surface corresponding to one of the above relations has Euler characteristic at most -2 , or it is a punctured torus.

Since the tower $G$ can be described as an iterated sequence of amalgamated products, its level groups $\mathrm{G}^{\ell}$ are naturally embedded in the $\omega$-residually free tower $\mathrm{G}$. From the definition of towers it is clear they admit a strict MR resolution, so by theorem 5.12 they are $\omega$-residually free groups. It is also easy to find their cyclic JSJ decomposition and their analysis lattice.

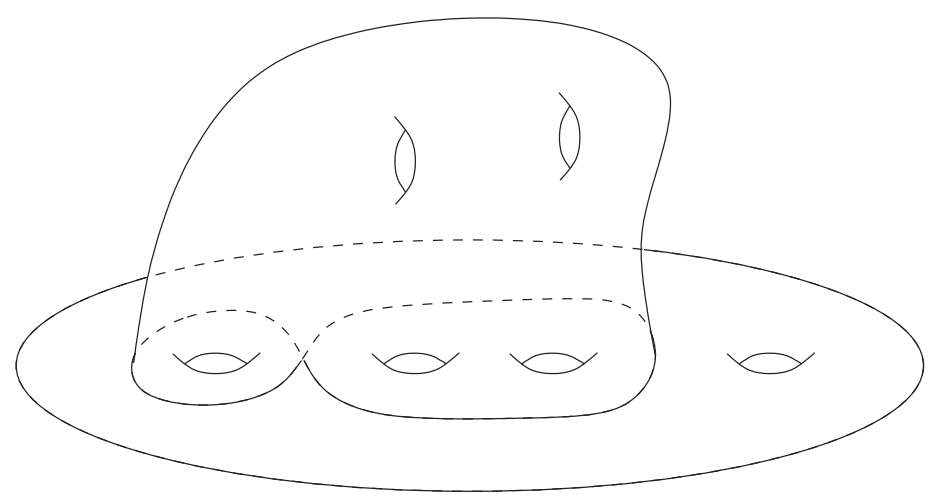


With an $\omega$-residually free tower $G$ we can naturally associate a 2-complex $\mathrm{T}(\mathrm{G})$, constructed hierarchically by adding a bouquet of circles for each free block, a collection of 2-tori for each Abelian block, and a (punctured) surface for each quadratic block. Clearly, $\pi_{1}(\mathrm{~T}(\mathrm{G}))=\mathrm{G}$.

\section{Residually Free Groups}

In the previous 6 sections we have studied the algebraic structure of limit groups which are $\omega$-residually free groups. When we get to study the structure of sets of solutions to equations in a free group in the next section, which we view as the analogue of algebraic varieties, $\omega$-residually free groups are going to serve as the (dual of the) analogue of irreducible varieties.

As we have already pointed out in the first section sets of solutions to a system of equations $\Phi$ in a free group $\mathrm{F}_{k}$ are equivalent to the set of all homomorphisms from the naturally associated group $\mathrm{G}(\Phi)$ into $\mathrm{F}_{k}$ (if the system $\Phi$ is with coefficients, we look only at homomorphisms that send the coefficients to the appropriate elements in $\mathrm{F}_{k}$ ). In studying all homomorphisms from $\mathrm{G}(\Phi)$ into a free group $\mathrm{F}_{k}$, we are not really recovering the algebraic structure of the group $G(\Phi)$ itself, but rather the algebraic structure of its residually free quotient.

Definition 7.1. - Let $\mathrm{G}$ be a f.g. group. We denote by $\mathrm{KF}(\mathrm{G})$ the intersection of all the kernels of homomorphisms from $\mathrm{G}$ into a free group. We call $\mathrm{RF}(\mathrm{G})=\mathrm{G} / \mathrm{KF}(\mathrm{G})$ the residually free quotient of $\mathrm{G}$.

Clearly, a residual free quotient is always a residually free group (see definition 4.5) and the residually free quotient of a residually free group $\mathrm{H}$ is the group $\mathrm{H}$ itself. Therefore, to understand the structure of residually free quotients of f.g groups, which is equivalent to the understanding of sets of solutions to equations in a free group, we need to study the algebraic structure of residually free groups. In this section we will use some of the arguments and results obtained in earlier sections on the structure of $\omega$-residually free groups, to recover the structure of residually free groups.

Let $\mathrm{RF}$ be a f.g. freely-indecomposable residually free group. On the set of ordered couples $\{(\tau, \mathrm{R})\}$, where $\mathrm{R}$ is an $\omega$-residually free quotient of $\mathrm{RF}$, and $\tau$ is an epimorphism $\tau: R F \rightarrow R$, we define an equivalence relation. We say that $\left(R_{1}, \tau_{1}\right)$ is equivalent to $\left(R_{2}, \tau_{2}\right)$ if there exists an isomorphism $v: R_{1} \rightarrow R_{2}$ so that $\tau_{2}=v \circ \tau_{1}$.

On the same set of ordered couples we also define a partial order. We say that $\left(R_{1}, \tau_{1}\right)>\left(R_{2}, \tau_{2}\right)$ if $R_{2}$ is a proper quotient of $R_{1}$ and there exists some epimorphism $\lambda: \mathrm{R}_{1} \rightarrow \mathrm{R}_{2}$ so that $\tau_{2}=\lambda \circ \tau_{1}$. 
An algebraic variety over an algebraically closed field is a union of irreducible varieties. The following, which is the main result of this section, is the analogue of this statement for varieties of solutions over a free group.

Theorem 7.2. - Let RF be a f.g. residually free group. Then there exists a (canonical) finite collection of $\omega$-residually free quotients of $\mathrm{RF}: \mathbf{R}_{1}, \ldots, \mathbf{R}_{\ell}$ and for each $i$ an epimorphism $\tau_{i}: \mathrm{RF} \rightarrow \mathrm{R}_{i}$ with the following properties:

(i) For each homomorphism $h: \mathrm{R} \rightarrow \mathrm{F}_{k}$ there exists some index $i$ (not necessarily unique!) and a homomorphism $f: \mathbf{R}_{i} \rightarrow \mathrm{F}_{k}$ so that $h$ splits as: $h=f \circ \tau_{i}$.

(ii) If $\mathrm{R}$ is an $\omega$-residually free quotient of $\mathrm{RF}$, and $\tau$ is an epimorphism $\tau: \mathrm{RF} \rightarrow \mathrm{R}$, then there exists some index $i$ and an epimorphism $\lambda: \mathrm{R}_{i} \rightarrow \mathrm{R}$ so that $\tau=\lambda \circ \tau_{i}$.

(iii) The couples $\left\{\left(\mathbf{R}_{i}, \tau_{i}\right)\right\}$ represent distinct equivalence classes, and if $i \neq j$ then the couple $\left(\mathbf{R}_{i}, \tau_{i}\right)$ can not be compared with $\left(\mathbf{R}_{j}, \tau_{j}\right)$ in the partial order defined on these couples.

(iv) If $\widehat{\mathrm{R}}_{1}, \ldots, \widehat{\mathrm{R}}_{u}$ and $\left\{\widehat{\tau}_{j}: \mathrm{RF} \rightarrow \widehat{\mathrm{R}}_{j}\right\}$ is another collection of $\boldsymbol{\omega}$-residually free quotients of $\mathrm{RF}$ and epimorphisms from $\mathrm{RF}$ to the $\widehat{\mathrm{R}}_{i}$ 's that satisfy properties (i)-(iii) then $u=\ell$ and up to permutation the couple $\left(\mathbf{R}_{i}, \tau_{i}\right)$ is equivalent to the couple $\left(\widehat{\mathbf{R}}_{i}, \widehat{\tau}_{i}\right)$.

Proof. - The collection of ordered couples $\{(\mathrm{R}, \tau)\}$ where $\mathrm{R}$ is an $\omega$-residually free quotient of $\mathrm{RF}$ and $\tau: \mathrm{RF} \rightarrow \mathrm{R}$ is an epimorphism, has similar properties to the partial order defined on shortening quotients of an $\omega$-residually free group in section 5 .

Lemma 7.3. - Let $\mathrm{RF}$ be a f.g. residually free group. Let $\left(\mathrm{R}_{1}, \tau_{1}\right),\left(\mathrm{R}_{2}, \tau_{2}\right),\left(\mathrm{R}_{3}, \tau_{3}\right), \ldots$, be a sequence of $\omega$-residually free quotients of $\mathrm{RF}$ and suppose $\left(\mathrm{R}_{1}, \tau_{1}\right)<\left(\mathrm{R}_{2}, \tau_{2}\right)<\left(\mathrm{R}_{3}, \tau_{3}\right)<\ldots$ is an infinite properly increasing sequence.

Then there exists an $\omega$-residually free quotient $\mathrm{R}$ of $\mathrm{RF}$ and an epimorphism $\tau: \mathrm{RF} \rightarrow \mathrm{R}$ so that $(\mathbf{R}, \tau)>\left(\mathbf{R}_{j}, \tau_{j}\right)$ for every $\omega$-residually free quotient $\left(\mathbf{R}_{j}, \tau_{j}\right)$ in the increasing sequence.

Proof. - Identical with the proof of lemma 5.4.

Lemma 7.3 proves the existence of maximal elements with respect to the partial order on the set of $\omega$-residually free quotients of the residually free group $\mathrm{R}$. The next lemma shows that there are only finitely many equivalence classes of maximal elements in the set of $\omega$-residually free quotients of RF.

Lemma 7.4. - Let $\mathrm{RF}$ be a f.g. residually free group. The set of ordered couples $\{(\mathrm{R}, \tau)\}$, where $\mathrm{R}$ is an $\omega$-residually free quotient of $\mathrm{RF}$ and $\tau: \mathrm{RF} \rightarrow \mathrm{R}$ is an epimorphism, contains only finitely many equivalence classes of maximal elements with respect to its partial order.

Proof. - Identical with the proof of lemma 5.5. 
Now, if $\left(\mathbf{R}_{1}, \tau_{1}\right), \ldots,\left(\mathbf{R}_{k}, \tau_{k}\right)$ are representatives for the distinct equivalence classes of maximal $\omega$-residually free quotients of the residually free group $R F$, then $\left(\mathrm{R}_{1}, \tau_{1}\right), \ldots,\left(\mathbf{R}_{k}, \tau_{k}\right)$ satisfy the conclusion of theorem 7.2 .

We will call the groups $\mathrm{R}_{1}, \ldots, \mathrm{R}_{k}$ together with the epimorphisms $\tau_{i}: \mathrm{RF} \rightarrow \mathrm{R}_{i}$ that appear in the formulation of theorem 7.2, the (canonical) (maximal) $\omega$-residually free groups associated with the f.g. residually free group RF. By theorem 7.2 these are canonically defined up to equivalence.

In section 5 we have associated a Makanin-Razborov diagram with each $\omega$-residually free group. This diagram was canonically defined and encodes all homomorphisms from an $\omega$-residually free group into free groups. Theorem 7.2 allows us to generalize the construction of the Makanin-Razborov diagram to residually free groups.

Let RF be a f.g. residually free group. We start with one vertex labeled RF and continue the diagram by adding an edge from this vertex to each of its associated maximal $\omega$-residually free quotients, and from each of the maximal $\omega$-residually free quotients we continue by adding its Makanin-Razborov diagram. By theorem 7.2 and the properties of the Makanin-Razborov diagram of $\omega$-residually free groups (see theorem 5.7), each homomorphism from the residually free group RF into some free group $\mathrm{F}_{k}$ can be "read" from the Makanin-Razborov diagram of RF.

In theorem 5.12 we have shown that a f.g. group is $\omega$-residually free if and only if it admits a strict MR resolution. Theorem 7.2 allows us to get corresponding classification of residually free groups. Note that a similar result is obtained in [Kh-My] (corollary 2).

Claim 7.5. - A f.g. group $\mathrm{G}$ is a residually free group if and only if $\mathrm{G}$ is a sub-direct product of a finite collection of $\omega$-residually free groups.

Proof. - Clearly, a sub-direct product of a finite collection of residually free groups is residually free, so one direction is obvious. The other direction of the claim follows from theorem 7.2, since a residually free group is a sub-direct product of its associated maximal $\omega$-residually free quotients.

Remarks.

(i) Corollary 4.4 shows that $\omega$-residually free groups are f.p. However f.g. residually free groups need not be f.p. If $\mathrm{F}_{g}$ is a free group then $\mathrm{F}_{g}+\mathrm{F}_{g}$ is residually free, so every subgroup of $\mathrm{F}_{g}+\mathrm{F}_{g}$ is residually free, and $\mathrm{F}_{g}+\mathrm{F}_{g}$ contains f.g. subgroups which are not f.p.

(ii) There is no global bound for the length of a sequence of proper residually free quotients of a residually free group. 


\section{Sets of Solutions to Equations in a Free Group}

In the first section we have pointed out that the sets of solutions to a system of equations $\Phi$ in a free group is equivalent to the sets of homomorphisms from a corresponding f.p. group $\mathrm{G}(\Phi)$ to a free group.

If the system of equations is without coefficients, then the sets of homomorphisms from $\mathrm{G}(\Phi)$ into a free group can be "read" from the Makanin-Razborov diagram corresponding to $G(\Phi)$ as was shown in the previous section. If the system $\Phi$ is with coefficient one needs to modify the whole theory developed in the previous sections to the presence of prescribed coefficients. The rest of this section is devoted to this modification.

Let $\Phi$ be a finite system of equations defined over a free group $\mathrm{F}_{k}=\left\langle a_{1}, \ldots, a_{k}\right\rangle$. Recall that with $\Phi$ it is natural to associate a f.p. group $G(\Phi)$. If the system $\Phi$ is defined by the coefficients $a_{1}, \ldots, a_{k}$, the unknowns $x_{1}, \ldots, x_{n}$ and the equations:

$$
\begin{gathered}
w_{1}\left(a_{1}, \ldots, a_{k}, x_{1}, \ldots, x_{n}\right)=1 \\
\vdots \\
w_{s}\left(a_{1}, \ldots, a_{k}, x_{1}, \ldots, x_{n}\right)=1
\end{gathered}
$$

we set the associated f.p. group $G(\Phi)$ to be:

$$
\mathrm{G}(\Phi)=<a_{1}, \ldots, a_{k}, x_{1}, \ldots, x_{n} \mid w_{1}, \ldots, w_{s}>
$$

Clearly, every solution of the system $\Phi$ corresponds to a homomorphism $h: \mathrm{G}(\Phi) \rightarrow \mathrm{F}_{k}$ for which $h\left(a_{i}\right)=a_{i}$, and every such homomorphism corresponds to a solution of the system $\Phi$. Therefore, the study of sets of solutions to systems of equations in a free group is equivalent to the study of all homomorphisms from a fixed f.p. group $\mathrm{G}$ into a free group $F_{k}$ for which a given prescribed set of elements in $G$ is mapped to a fixed basis of the free group $\mathrm{F}_{k}$.

For the rest of this section, we set $\mathrm{G}$ to be a f.g. group $\mathrm{G}=\left\langle g_{1}, \ldots, g_{m}\right\rangle$, $\gamma_{1}, \ldots, \gamma_{k} \in \mathrm{G}$ to be a prescribed set of elements in $\mathrm{G}$, and $\mathrm{F}_{k}$ to be a free group with a fixed basis $\mathrm{F}_{k}=\left\langle a_{1}, \ldots, a_{k}\right\rangle$. With these notation our main goal is to get a structure theory for understanding the set of all homomorphisms:

$$
\operatorname{Hom}\left(\left(\mathrm{G},\left\{\gamma_{i}\right\}\right),\left(\mathrm{F}_{k},\left\{a_{i}\right\}\right)\right)=\left\{h \mid h: \mathrm{G} \rightarrow \mathrm{F}_{k}, h\left(\gamma_{i}\right)=a_{i}\right\}
$$

which for brevity we will denote $\operatorname{Hom}\left(\mathrm{G}, \mathrm{F}_{k}\right)$. We set $\mathrm{X}$ to be the Cayley graph of $\mathrm{F}_{k}$ with respect to its given basis $a_{1}, \ldots, a_{k}$. Given any homomorphism $h \in \operatorname{Hom}\left(\mathrm{G}, \mathrm{F}_{k}\right), \mathrm{G}$ admits a natural action $\lambda_{h}$ on $\mathrm{X}$ given by $\lambda_{h}(g, x)=h(g)(x)$ for every $g \in \mathrm{G}$ and $x \in \mathrm{X}$.

Let $\left\{h_{j}\right\} \subset \operatorname{Hom}\left(\mathrm{G}, \mathrm{F}_{k}\right)$ be a set of distinct homomorphisms from $\mathrm{G}$ to $\mathrm{F}_{k}$ satisfying the prescribed conditions. We set $\mu_{j}$ to be: $\mu_{j}=\max _{1 \leqslant u \leqslant m} d_{\mathrm{X}}\left(i d\right.$.,$h_{j}\left(g_{u}\right)$ ) (note that unlike what we did in section 1, here, due to the presence of coefficients, we 
do not compose with an inner automorphism of $\mathrm{F}_{k}$ ). Since the homomorphisms in the sequence $\left\{h_{j}\right\} \subset \operatorname{Hom}\left(\mathrm{G}, \mathrm{F}_{k}\right)$ are distinct, the sequence of stretching factors $\left\{\boldsymbol{\mu}_{j}\right\}$ does not contain a bounded subsequence. We set $\left\{\left(\mathrm{X}_{j}, x_{j}\right)\right\}_{j=1}^{\infty}$ to be the pointed metric spaces obtained by rescaling the metric on the Cayley graph of $\mathrm{F}_{k},(\mathrm{X}, i d$.$) , by \mu_{j} .\left(\mathrm{X}_{j}, x_{j}\right)$ is endowed with a left isometric action of our f.g. group $\mathrm{G}$ via the homomorphisms $\left\{h_{j}\right\}$. This sequence of actions of $\mathrm{G}$ on the metric spaces $\left\{\left(\mathrm{X}_{j}, x_{j}\right)\right\}_{j=1}^{\infty}$ allows us to obtain an action of $\mathrm{G}$ on a real tree $\left(\mathrm{Y}, y_{0}\right)$ by passing to a Gromov-Hausdorff limit of a convergent subsequence (proposition 1.1).

With the limit tree we obtained by using the Gromov-Hausdorff topology we associate natural algebraic objects, the kernel of the action of $\mathrm{G}$ on this (limit) real tree and the quotient of $\mathrm{G}$ by this kernel which we call the restricted limit group. We will denote the restricted limit group by $\mathrm{RL}_{\infty}$, and the canonical quotient map by $\eta: G \rightarrow \mathrm{RL}_{\infty}$. Note that since a restricted limit group is a limit group in the sense of section 1, all the algebraic properties of limit groups hold for restricted limit groups. Still, for the purpose of understanding the set of all restricted homomorphisms from $\mathrm{G}$ to the (fixed) free group $\mathrm{F}_{k}$ we will need to modify some of the algebraic objects associated in the previous sections with limit groups, to study restricted limit groups.

The abelian JSJ decomposition of a freely-indecomposable limit group "encodes" all the possible splittings of such limit group with abelian edge groups. In studying restricted limit groups we will be interested only abelian splittings in which the subgroup $<\eta\left(\gamma_{1}\right), \ldots, \eta\left(\gamma_{k}\right)>$ is elliptic. The construction of the abelian JSJ decomposition of a limit group described in section 2, naturally generalizes to a restricted abelian JSJ decomposition of a restricted limit group.

Theorem 8.1 (cf. theorem 2.7). - Suppose $\mathrm{RL}_{\infty}$ is a restricted limit group which does not split to a non-trivial free decomposition in which the subgroup $\eta(\Gamma)=\left\langle\eta\left(\gamma_{1}\right), \ldots, \eta\left(\gamma_{k}\right)\right\rangle$ is contained in one of the factors. Suppose that $\eta(\Gamma)$ is a proper subgroup of $\mathrm{RL}_{\infty}$. Then there exists a reduced unfolded splitting of $\mathrm{RL}_{\infty}$ with abelian edge groups, which we call an abelian restricted JSJ (Jaco-Shalen-Fohannson) decomposition of $\mathrm{RL}_{\infty}$ with the following properties:

(i) $\eta(\Gamma)$ is elliptic in the restricted abelian JSJ decomposition.

(ii) Every (restricted) canonical maximal $\mathrm{QH}$ subgroup (CMQ) of $\mathrm{RL}_{\infty}$ is conjugate to a vertex group in the JSJ decomposition. Every (restricted) $\mathrm{QH}$ subgroup of $\mathrm{RL}_{\infty}$ can be conjugated into one of the $\mathrm{CMQ}$ subgroups of $\mathrm{RL}_{\infty}$. Every vertex group in the JSJ decomposition which is not a CMQ subgroup of $\mathrm{RL}_{\infty}$ is elliptic in any abelian splitting of $\mathrm{RL}_{\infty}$ under consideration (i.e., in one in which $\eta(\Gamma)$ is elliptic).

(iii) A one edge abelian splitting $\mathrm{RL}_{\infty}=\mathrm{D} *_{\mathrm{A}} \mathrm{E}$ or $\mathrm{RL}_{\infty}=\mathrm{D} *_{\mathrm{A}}$ in which $\eta(\Gamma)$ is elliptic which is hyperbolic in another such elementary abelian splitting is obtained from the restricted abelian JSJ decomposition of $\mathrm{RL}_{\infty}$ by cutting a 2-orbifold corresponding to a CMQ subgroup of $\mathrm{RL}_{\infty}$ along a weakly essential s.c.c. 
(iv) Let $\Theta$ be a one edge splitting along an abelian subgroup $\mathrm{A}, \mathrm{RL}_{\infty}=\mathrm{D} *_{\mathrm{A}} \mathrm{E}$ or $\mathrm{RL} \infty_{\infty}=\mathrm{D} *_{\mathrm{A}}$ in which $\eta(\Gamma)$ is elliptic, so that $\mathrm{A}$ is elliptic with respect to any other one edge abelian splitting of $\mathrm{RL}_{\infty}$ in which $\eta(\Gamma)$ is elliptic. Then $\Theta$ is obtained from the restricted JSJ decomposition of $\mathrm{RL}_{\infty}$ by a sequence of collapsings, foldings, and conjugations.

(v) If $\mathrm{JSJ}_{1}$ is another restricted abelian JSJ decomposition of $\mathrm{RL}_{\infty}$, then $\mathrm{JSJ}_{1}$ is obtained from the restricted abelian JSJ decomposition by a sequence of slidings, conjugations and modifying boundary monomorphisms by conjugations (see section 1 of [Ri-Se2] for these notions).

From the canonical properties of the abelian JSJ decomposition of a limit group we were able to show in section 3 that a non-abelian, freely indecomposable limit group admits a principal cyclic splitting. The arguments used in proving theorem 3.2 naturally generalize to prove the existence of a (restricted) principal cyclic splitting of a restricted limit group.

Theorem 8.2. — Let $\mathrm{RL}_{\infty}$ be a restricted limit group which does not split to a non-trivial free product in which the subgroup $\eta(\Gamma)$ is contained in one of the factors. Suppose that $\eta(\Gamma)$ is a proper subgroup of $\mathrm{RL}_{\infty}$. Then there exists a principal cyclic splitting of $\mathrm{RL}_{\infty}$ in which $\eta(\Gamma)$ is elliptic.

The restricted abelian JSJ decomposition together with the existence of a restricted principal splitting allow us to generalize the arguments of section 3 to construct the restricted cyclic JSJ decomposition of a restricted limit group (cf. theorem 3.9).

Since a restricted limit group is in particular a limit group, it is finitely presented (in fact, it is also possible to modify the construction of the analysis lattice to the restricted setup). Like in the case of limit groups, the finite presentability of restricted limit groups also shows that restricted limit groups are restricted $\omega$-residually free groups. i.e., that for every finite set of elements in a restricted limit group $\mathrm{RL}_{\infty}$ there exists a restricted homomorphism $h: \mathrm{RL}_{\infty} \rightarrow \mathrm{F}_{k}$ that maps the elements in this finite set to distinct elements in $\mathrm{F}_{k}$.

In section 5 we have used the canonical cyclic JSJ decomposition of a limit group to construct its canonical Makanin-Razborov diagram. We continue by modifying the notions and arguments appear in section 5 to construct the restricted MakaninRazborov diagram of a restricted limit group.

Definition 8.3. - Let $\mathrm{RL}_{\infty}$ be a restricted limit group which does not split to a non-trivial free product in which the subgroup $\eta(\Gamma)$ is contained in one of the factors. Suppose that $\eta(\Gamma)$ is a proper subgroup of $\mathrm{RL}_{\infty}$. We define the restricted modular group $R M o d\left(\mathrm{RL}_{\infty}\right)$ to be the subgroup of $A u t(\mathbf{R})$ generated by the following families of automorphisms of $\mathbf{R}$ : 
(i) Dehn twists along edges of the restricted cyclic JSJ decomposition of $\mathrm{RL}_{\infty}$. The Dehn twists are assumed to fix (elementwise) the vertex stabilized by $\eta(\Gamma)$.

(ii) Dehn twists along essential s.c.c. in GMQ (canonical maximal quadratically hanging) vertex groups in the restricted cyclic JSJ decomposition of $\mathrm{RL}_{\infty}$. Again, these Dehn twists are assume to fix (elementwise) the vertex stabilized by $\eta(\Gamma)$.

(iii) Let $\mathrm{A}$ be an abelian vertex group in the restricted cyclic JSJ decomposition of $\mathrm{RL}_{\infty}$. Let $\mathrm{A}_{1}<\mathrm{A}$ be the subgroup generated by all edge groups connected to the vertex stabilized by $\mathrm{A}$ in the cyclic JSJ decomposition of $\mathrm{R}$. Every automorphism of $\mathrm{A}$ that fixes $\mathrm{A}_{1}$ (elementwise) can be naturally extended to an automorphism of the ambient limit group $\mathrm{RL}_{\infty}$ that fixes the vertex stabilized by $\eta(\Gamma)$. We call these generalized Dehn twists and they form the third family of automorphisms that generate $\operatorname{RMod}(\mathbf{R})$.

Given a sequence of homomorphisms of a limit group into a free group, we used the modular group of automorphisms of that limit group to modify the sequence of homomorphisms and obtain a shortening quotient of the limit group we started with. Given a restricted limit group, and a sequence of homomorphisms of that restricted limit group into $\mathrm{F}_{k}$, we may use the restricted modular group of automorphisms of that restricted limit group to modify the sequence of homomorphisms and obtain a restricted shortening quotient of a restricted limit group. By the same argument used to prove claim 5.3 a restricted shortening quotient of a restricted limit group is a proper quotient of it.

On the set of restricted shortening quotients of a restricted limit group we can naturally define a partial order and an equivalence relation, similar to the ones defined in section 5 for shortening quotients of limit groups. By the same argument used to prove lemma 5.4 the set of restricted shortening quotients of a restricted limit group contains maximal elements with respect to the partial order, and by the same argument used to prove lemma 5.5 there are only finitely many equivalence classes of maximal restricted shortening quotients of a restricted limit group. Like in proposition 5.6 and using an identical argument, for every restricted homomorphism $\mathrm{RL}_{\infty} \rightarrow \mathrm{F}_{k}$ there exists some index $1 \leqslant i \leqslant k$ (not necessarily unique!) and a restricted modular automorphism $\varphi_{h} \in R \operatorname{Mod}(\mathrm{R})$ so that $h \circ \varphi_{h}$ splits through the maximal restricted shortening quotient $\mathrm{RM}_{i}$. I.e., $h \circ \varphi_{h}=h_{\mathrm{R}_{i}} \circ \eta_{i}$ where $h_{\mathrm{R}_{i}}: \mathrm{R}_{i} \rightarrow \mathrm{F}_{k}$ is a restricted homomorphism.

These basic facts concerning restricted limit groups and their restricted shortening quotients allow us to present the main goal of this section, the (canonical) restricted Makanin-Razborov diagram associated with a restricted limit group (restricted $\omega$-residually free group). The restricted Makanin-Razborov diagram "encodes" all possible restricted homomorphisms from a limit group into the (fixed) free group $\mathrm{F}_{k}$, and like for limit groups some of its properties can be stated as a criteria for a general f.g. group to be a restricted limit group.

Let $\mathrm{RL}_{\infty}$ be a restricted limit group (a restricted $\omega$-residually free group). By definition in $\mathrm{RL}_{\infty}$ there exists a (distinguished) finite set of elements $\left\langle\gamma_{1}, \ldots, \gamma_{k}\right\rangle$ so 
that for any finite set $\mathrm{U}$ of elements in $\mathrm{RL}_{\infty}$ there exists a (restricted) homomorphism $h: \mathrm{RL}_{\infty} \rightarrow \mathrm{F}_{k}$ that sends $\gamma_{i}$ to $a_{i}$, the $i$-th basis element of $\mathrm{F}_{k}$, and sends the elements of $\mathrm{U}$ to distinct elements of $\mathrm{F}_{k}$. Let $\Gamma=\left\langle\gamma_{1}, \ldots, \gamma_{k}\right\rangle$.

Let $\mathrm{RL}_{\infty}=\mathrm{RL}_{1} * \ldots \mathrm{RL}_{\ell} * \mathrm{~F}_{g_{1}}$ be a factorization of $\mathrm{R}$, where $\Gamma<\mathrm{RL}_{1}, \mathrm{RL}_{1}$ can not be written as a free product in which $\Gamma$ is contained in one of the factors, each of the $\mathrm{RL}_{i}$ 's for $2 \leqslant i \leqslant \ell$ is a freely-indecomposable non-cyclic subgroup of $\mathrm{RL}$ and $\mathrm{F}_{g_{1}}$ is a free group. It follows from Grushko's theorem that such a free decomposition of $\mathrm{RL}_{\infty}$ is canonical. By an analogue of lemma 5.4 to the restricted case, the set of restricted shortening quotients of the restricted limit group $\mathrm{RL}_{1}$ contains maximal elements (with respect to the partial order defined above) and by an analogue of lemma 5.5 to the restricted case there are only finitely many equivalence classes of maximal restricted shortening quotients of $\mathrm{RL}_{1}$. Let $\mathrm{R}_{1}, \ldots, \mathrm{R}_{s}$ be a collection of representatives of equivalence classes of maximal restricted shortening quotients of $\mathrm{RL}_{1}$, and let $\eta_{j}: \mathrm{RL}_{1} \rightarrow \mathrm{M}_{j}$ be the canonical projection maps.

We define the restricted Makanin-Razborov diagram of the restricted limit group $\mathrm{RL}_{\infty}$ iteratively. We start by factoring $\mathrm{RL}_{\infty}$ as above. From each of the factors $\mathrm{RL}_{2}, \ldots, \mathrm{RL}_{\ell}$ we continue by adjoining its Makanin-Razborov diagram presented in section 5. From $\mathrm{RL}_{1}$ we continue along $s$ edges going from $\mathrm{RL}_{1}$ to each of its maximal restricted quotients $\mathrm{R}_{1}, \ldots, \mathrm{R}_{s}$. To each such directed edge we associate the canonical projection $\eta_{j}$.

We proceed iteratively. Since a restricted shortening quotient of a restricted limit group is a proper quotient of it, the construction terminates. Each terminal vertex is either a free group (as in the Makanin-Razborov diagram presented in section 5) or it is the (image of the) "distinguished" free group $\Gamma=\left\langle\gamma_{1}, \ldots, \gamma_{k}\right\rangle$. At least one of the terminal vertices in a restricted Makanin-Razborov diagram must be labeled with an image of the "distinguished" subgroup $\Gamma$. By construction, up to the equivalence relation of restricted shortening quotients, the restricted Makanin-Razborov diagram of a restricted limit group is canonical. By an analogue of theorem 5.7 to the restricted case, all the restricted homomorphisms from a restricted limit group $\mathrm{RL}_{\infty}$ into the free group $\mathrm{F}_{k}$ are encoded by the canonical restricted Makanin-Razborov diagram. I.e., they are all given by compositions of (restricted) modular automorphisms of the (restricted) limit groups in the diagram with the canonical maps from the (restricted) limit groups into their maximal (restricted) shortening quotients and finally with either the fixed map from (an image of) the distinguished subgroup $\Gamma$ onto $\mathrm{F}_{k}$, or general homomorphisms ("substitutions") of the terminal (non-distinguished) free groups appear in the diagram into the target free group $\mathrm{F}_{k}$.

The (canonical) restricted Makanin-Razborov diagram associated with a restricted limit group encodes all the restricted homomorphisms from that restricted limit group into a free group $\mathrm{F}_{k}$. Like in the non-restricted case (definitions 5.8 and 5.9) using the restricted Makanin-Razborov diagram we can define restricted Makanin-Razborov 
resolutions and restricted strict Makanin-Razborov resolutions of a restricted limit group. Like in the non-restricted case (theorem 5.12) the existence of a restricted strict MR resolution for a f.g. group $G$ with a distinguished subgroup $\Gamma=\left\langle\gamma_{1}, \ldots, \gamma_{k}\right\rangle$ is a criteria for $\mathrm{G}$ to be a restricted limit group (restricted $\omega$-residually free group). The proofs are all direct modifications of the ones given in section 5 in the non-restricted case.

Like restricted limit groups (restricted $\omega$-residually free groups) we may define in a similar way restricted residually free groups. The study of sets of solutions to equations with coefficients is naturally equivalent to study of all restricted homomorphisms from a f.g. restricted residually free group into the free group generated by the coefficients. For studying this collection of restricted homomorphisms we have an analogue of theorem 7.2. The proof is a direct modification.

Theorem 8.4. - Let $\mathrm{RF}$ be a f.g. restricted residually free group. Then there exists a (canonical) finite collection of restricted $\omega$-residually free quotients of $\mathrm{RF}: \mathbf{R}_{1}, \ldots, \mathbf{R}_{\ell}$ and for each $i$ an epimorphism $\tau_{i}: \mathrm{RF} \rightarrow \mathrm{R}_{i}$ with the following properties:

(i) For each restricted homomorphism $h: \mathrm{R} \rightarrow \mathrm{F}_{k}$ there exists some index $i$ (not necessarily unique!) and a restricted homomorphism $f: \mathbf{R}_{i} \rightarrow \mathbf{F}_{k}$ so that $h$ splits as: $h=f \circ \tau_{i}$.

(ii) If $\mathrm{R}$ is a restricted $\omega$-residually free quotient of $\mathrm{RF}$, and $\tau$ is an epimorphism $\tau: \mathrm{RF} \rightarrow \mathrm{R}$, then there exists some index $i$ and an epimorphism $\lambda: \mathrm{R}_{i} \rightarrow \mathrm{R}$ so that $\tau=\lambda \circ \tau_{i}$.

(iii) The couples $\left\{\left(\mathbf{R}_{i}, \tau_{i}\right)\right\}$ represent distinct equivalence classes, and if $i \neq j$ then the couple $\left(\mathbf{R}_{i}, \tau_{i}\right)$ can not be compared with $\left(\mathbf{R}_{j}, \tau_{j}\right)$ in the partial order defined on these couples.

(iv) If $\widehat{\mathbf{R}}_{1}, \ldots, \widehat{\mathbf{R}}_{u}$ and $\left\{\widehat{\tau}_{j}: \mathrm{RF} \rightarrow \widehat{\mathbf{R}}_{j}\right\}$ is another collection of restricted $\omega$-residually free quotients of $\mathrm{RF}$ and epimorphisms from $\mathrm{RF}$ to the $\widehat{\mathbf{R}}_{i}$ 's that satisfy properties (i)-(iii) then $u=\ell$ and up to permutation the couple $\left(\mathbf{R}_{i}, \tau_{i}\right)$ is equivalent to the couple $\left(\widehat{\mathbf{R}}_{i}, \widehat{\tau}_{i}\right)$.

We have already modified the construction of the Makanin-Razborov diagram of a $\omega$-residually free group to the restricted case. Having such diagram for restricted $\omega$ residually free group, theorem 8.4 allows us to modify the construction of the MakaninRazborov diagram associated with a residually free group presented in section 7 to the restricted case. This restricted Makanin-Razborov diagram of restricted residually free groups finally concludes our study of the structure of sets of solutions to systems of equations with coefficients. 


\section{Graded Limit Groups}

In the previous sections we have analyzed the structure of sets of solutions to systems of equations in a free group by studying the algebraic structure of limit groups which turned out to be $\omega$-residually free groups. To understand the elementary theory of free groups and, in particular, to study the structure of sets defined by elementary predicates (elementary sets), we will need to study the structure of sets of solutions to systems of equations with parameters, i.e., we will examine how the (restricted) Makanin-Razborov diagrams and resolutions vary with a change of the values of the defining parameters.

With a finite system of equations with parameters $\Phi$ over a free group $\mathrm{F}_{k}=\left\langle a_{1}, \ldots, a_{k}\right\rangle$ it is natural to associate a f.p. group $\mathrm{G}(\Phi)$. If the system $\Phi$ is defined by the coefficients $a_{1}, \ldots, a_{k}$, the parameters $p_{1}, \ldots, p_{u}$, the unknowns $x_{1}, \ldots, x_{n}$ and the equations:

$$
\begin{gathered}
w_{1}\left(a_{1}, \ldots, a_{k}, p_{1}, \ldots, p_{u}, x_{1}, \ldots, x_{n}\right)=1 \\
\vdots \\
w_{s}\left(a_{1}, \ldots, a_{k}, p_{1}, \ldots, p_{u}, x_{1}, \ldots, x_{n}\right)=1
\end{gathered}
$$

we set the associated f.p. group $\mathrm{G}(\Phi)$ to be:

$$
\mathrm{G}(\Phi)=<a_{1}, \ldots, a_{k}, p_{1}, \ldots, p_{u}, x_{1}, \ldots, x_{n} \mid w_{1}, \ldots, w_{s}>
$$

Clearly, every solution of the system $\Phi$ corresponds to a homomorphism $h: \mathrm{G}(\Phi) \rightarrow \mathrm{F}_{k}$ for which $h\left(a_{i}\right)=a_{i}$, and every such homomorphism corresponds to a solution of the system $\Phi$. Therefore, the study of sets of solutions to systems of equations in a free group is equivalent to the study of all homomorphisms from a fixed f.p. group $G$ into a free group $F_{k}$ for which a given prescribed set of elements in $G$ is mapped to a fixed basis of the free group $\mathrm{F}_{k}$.

In the previous sections we studied the global set of (restricted) homomorphisms from a f.g. group $\mathrm{G}$ into a free group $\mathrm{F}_{k}$, and later showed the existence of effective tools which enabled us to study the structure of this global set. In studying parametric systems of equations we will analyze the variation of this structure with a change of the defining parameters. Our whole study of the global set of restricted homomorphisms was based on understanding the algebraic structure of limit groups ( $\omega$-residually free groups). To analyze the variation in this structure with a change of the parameters we will need to look at a special class of limit groups which we call graded limit groups. Our study of graded limit groups is based on the tools we developed for studying (restricted) limit groups. However, as we will see in the sequel, not all the results we have obtained for (restricted) limit groups have analogues for graded limit groups, and the treatment of the new phenomenas that appear in studying graded limit groups require new notions and techniques. 
We set $\left.\mathrm{G}=<x_{1}, \ldots, x_{k}, p_{1}, \ldots, p_{u}, a_{1}, \ldots, a_{k}\right\rangle$ to be a f.g. group. For brevity we will denote this (fixed) generating set by $\mathrm{G}=\left\langle g_{1}, \ldots, g_{m}\right\rangle$, and $\mathrm{F}_{k}$ to be a free group with a fixed basis $\mathrm{F}_{k}=\left\langle a_{1}, \ldots, a_{k}\right\rangle$. With this notation our goal is to get a structure theory for understanding the set of all homomorphisms:

$$
\operatorname{Hom}\left(\left(\mathrm{G},\left\{p_{j}\right\},\left\{a_{i}\right\}\right),\left(\mathrm{F}_{k},\left\{a_{i}\right\}\right)\right)=\left\{h \mid h: \mathrm{G} \rightarrow \mathrm{F}_{k}, h\left(a_{i}\right)=a_{i}\right\}
$$

with a change in the values of the parameters $\left\{p_{j}\right\}$. We set $\mathrm{X}$ to be the Cayley graph of $\mathrm{F}_{k}$ with respect to its given basis $a_{1}, \ldots, a_{k}$. Given any homomorphism $h \in \operatorname{Hom}\left(\mathrm{G}, \mathrm{F}_{k}\right)$, $\mathrm{G}$ admits a natural action $\lambda_{h}$ on $\mathrm{X}$ given by $\lambda_{h}(g, x)=h(g)(x)$ for every $g \in \mathrm{G}$ and $x \in \mathrm{X}$.

Definition 9.1. - Let $\mathrm{G}$ be a f.g. group and let $h_{n}: \mathrm{G}(x, p, a) \rightarrow \mathrm{F}_{k}$ be a sequence of homomorphisms. If $\left\{\lambda_{h_{n}}\right\}$, a sequence of actions of $\mathrm{G}$ on $\mathrm{X}$, the Cayley graph of $\mathrm{F}_{k}$, that corresponds to the sequence of homomorphisms $\left\{h_{n}\right\}$, converges in the Gromov-Haussdorff topology, we say that the sequence $\left\{h_{n}\right\}$ converges. We call the obtained limit group, a graded limit group.

A graded limit group is in particular a limit group, so every f.g. graded limit group is f.p. and every f.g. graded limit group is $\omega$-residually free by theorem 4.6. To construct the (canonical) graded Makanin-Razborov diagram of a graded limit group which is the fundamental tool in our analysis of the variation of sets of solutions to systems of equations with parameters, we need to study the algebraic structure of graded limit groups "relative" to their grading. To do that we need to construct the graded abelian JSJ decomposition of a graded limit group, and the graded analysis lattice of a graded limit group. These constructions are all generalizations of their restricted analogues presented in section 8 .

For the rest of this section let $\mathrm{G}(x, p, a)$ be a f.g. group, let $\left\{h_{m}: \mathrm{G}(x, p, a) \rightarrow\right.$ $\left.\mathrm{F}_{k}\right\}$ be a convergent graded sequence of homomorphisms, and let $\operatorname{Glim}(x, p, a)$ be the corresponding graded limit group. We set $\eta: \mathrm{G}(x, p, a) \rightarrow \operatorname{Glim}(x, p, a)$ to be the canonical epimorphism and $\mathrm{AP}<\operatorname{Glim}(x, p, a)$ to be the subgroup: $\mathrm{AP}=<\eta\left(p_{1}\right), \ldots, \eta\left(p_{u}\right), \eta\left(a_{1}\right), \ldots, \eta\left(a_{k}\right)>$. The abelian JSJ decomposition of a freelyindecomposable limit group "encodes" all the possible splittings of such limit group with abelian edge groups. In studying graded limit groups we will be interested only in abelian splittings in which the subgroup AP is elliptic. The construction of the abelian JSJ decomposition of a limit group described in section 2 naturally generalizes to a graded abelian JSJ decomposition of a graded limit group.

Theorem 9.2 (cf. 8.1). - Suppose Glim $(x, p, a)$ is a graded limit group which does not split to a non-trivial free decomposition in which the subgroup $\mathrm{AP}$ is contained in one of the factors. Then there exists a (relatively) reduced unfolded (perhaps trivial) splitting of $\operatorname{Glim}(x, p, a)$ with abelian edge groups, which we call a graded abelian JSJ (Jaco-Shalen-Fohannson) decomposition of $\operatorname{Glim}(x, p, a)$ with the following properties: 
(i) AP is elliptic in the graded abelian JSJ decomposition.

(ii) Every (graded) canonical maximal $\mathrm{QH}$ subgroup (CMQ) of Glim $(x, p, a)$ is conjugate to a vertex group in the graded JSJ decomposition. Every (graded) $\mathrm{QH}$ subgroup of $\operatorname{Glim}(x, p, a)$ can be conjugated into one of the CMQ subgroups of $\operatorname{Glim}(x, p, a)$. Every vertex group in the graded JSJ decomposition which is not a CMQ subgroup of $\operatorname{Glim}(x, p, a)$ is elliptic in any abelian splitting of $\operatorname{Glim}(x, p, a)$ under consideration (i.e., in one in which AP is elliptic).

(iii) A one edge (graded) abelian splitting $\operatorname{Glim}(x, p, a)=\mathrm{D} *_{\mathrm{A}} \mathrm{E}$ or $\operatorname{Glim}(x, p, a)=\mathrm{D} *_{\mathrm{A}}$ in which $\mathrm{AP}$ is elliptic which is hyperbolic in another such elementary abelian splitting is obtained from the graded abelian JSJ decomposition of $\operatorname{Glim}(x, p, a)$ by cutting a 2-orbifold corresponding to a (graded) CMQ subgroup of $\operatorname{Glim}(x, p, a)$ along a weakly essential s.c.c.

(iv) Let $\Theta$ be a one edge (graded) splitting along an abelian subgroup $\operatorname{Glim}(x, p, a)=\mathrm{D} *_{\mathrm{A}} \mathrm{E}$ or $\operatorname{Glim}(x, p, a)=\mathrm{D} *_{\mathrm{A}}$ in which $\mathrm{AP}$ is elliptic, which is elliptic with respect to any other one edge (graded) abelian splitting of $\operatorname{Glim}(x, p, a)$ in which $\mathrm{AP}$ is elliptic. Then $\Theta$ is obtained from the graded JSJ decomposition of $\operatorname{Glim}(x, p, a)$ by a sequence of collapsings, foldings and conjugations.

(v) If $\mathrm{JSJ}_{1}$ is another graded abelian JSJ decomposition of $\operatorname{Glim}(x, p, a)$, then $\mathrm{JSJ}_{1}$ is obtained from the graded abelian JSJ decomposition by a sequence of slidings, conjugations and modifying boundary monomorphisms by conjugations (see section 1 of [Ri-Se2] for these notions).

\section{Graded Makanin-Razborov Resolutions and Diagram}

In section 5 we have used the canonical cyclic JSJ decomposition of a limit group to construct its canonical Makanin-Razborov diagram. In section 8 we modified this construction to obtain the restricted Makanin-Razborov diagram for analyzing sets of solutions to equations with coefficients. To understand the variation of the restricted Makanin-Razborov diagram with a change of the defining parameters we need to further modify the construction of the Makanin-Razborov diagram to obtain the graded Makanin-Razborov diagram of a graded limit group. As we have already indicated, some of the results that were essential in the construction of the (ungraded) Makanin-Razborov diagram do not hold when graded limit groups and their graded decompositions are concerned. Hence, to construct the graded Makanin-Razborov diagram of a graded limit group, some new types of graded limit groups that do not have analogues in the ungraded case are presented, and the structure of the graded diagram as a whole requires far more delicate analysis than in the ungraded case. This analysis is presented in the rest of this paper and in the third paper of this sequence.

Definition 10.1. - Let Glim $(x, p, a)$ be a graded limit group which does not split to a nontrivial free product in which the subgroup $\mathrm{AP}=\left\langle p_{1}, \ldots, p_{u}, a_{1}, \ldots, a_{k}\right\rangle$ is contained in one of the 
factors. Suppose that the principal graded abelian JSJ decomposition of $\operatorname{Glim}(x, p, a)$ is non-trivial. We define the graded modular group $\operatorname{GMod}(\operatorname{Glim}(x, p, a))$ to be the subgroup of $\operatorname{Aut}(\operatorname{Glim}(x, p, a))$ generated by the following families of automorphisms of $\operatorname{Glim}(x, p, a)$ :

(i) Dehn twists along edges of the principal graded abelian JSJ decomposition of $\operatorname{Glim}(x, p, a)$. The Dehn twists are assumed to fix (elementwise) the vertex stabilized by AP.

(ii) Dehn twists along essential s.c.c. in (graded) GMQ (canonical maximal quadratically hanging) vertex groups in the principal graded abelian JSJ decomposition of $\operatorname{Glim}(x, p, a)$. Again, these Dehn twists are assume to fix (elementwise) the vertex stabilized by AP.

(iii) Let A be an abelian vertex group in the principal graded abelian JSJ decomposition of $\operatorname{Glim}(x, p, a)$. Let $\mathrm{A}_{1}<\mathrm{A}$ be the subgroup generated by all edge groups connected to the vertex stabilized by $\mathrm{A}$ in the principal graded abelian JSJ decomposition of $\operatorname{Glim}(x, p, a)$. Every automorphism of $\mathrm{A}$ that fixes $\mathrm{A}_{1}$ (elementwise) can be naturally extended to an automorphism of the ambient limit group $\operatorname{Glim}(x, p, a)$ that fixes the vertex stabilized by AP. We call these generalized Dehn twists and they form the third family of automorphisms that generate $\operatorname{GMod}(\operatorname{Glim}(x, p, a))$.

We say that a homomorphism $h: \operatorname{Glim}(x, p, a) \rightarrow \mathrm{F}_{k}$ is in the graded modular class of the homomorphism $\widehat{h}: \operatorname{Glim}(x, p, a) \rightarrow \mathrm{F}_{k}$ if $\widehat{h}=h \circ \varphi$, where $\varphi$ is a graded modular automorphism of $\operatorname{Glim}(x, p, a)$, i.e., $\varphi \in \operatorname{GMod}(\operatorname{Glim}(x, p, a))$. Clearly, a graded modular class is an equivalence class of homomorphisms from the graded modular group Glim $(x, p, a)$ to the free group $\mathrm{F}_{k}$.

Let $\operatorname{Glim}(x, p, a)$ be a graded limit group that admits a non-trivial graded abelian JSJ decomposition. Once we fix a generating set for the graded limit group $\operatorname{Glim}(x, p, a)$, given a homomorphism $h: \operatorname{Glim}(x, p, a) \rightarrow \mathrm{F}_{k}$ we can choose the shortest homomorphisms in the graded modular class of $h$. A graded limit group obtained from a convergent sequence of homomorphisms $h: \operatorname{Glim}(x, p, a) \rightarrow \mathrm{F}_{k}$ which are the shortest in their graded modular class, is called a graded shortening quotient of the graded limit group $\operatorname{Glim}(x, p, a)$.

On the set of graded shortening quotients of a graded limit group we can naturally define a partial order and an equivalence relation, similar to the ones defined in section 5 for shortening quotients of limit groups. By the same argument used to prove lemma 5.4 the set of graded shortening quotients of a graded limit group contains maximal elements with respect to the partial order, and by the same argument used to prove lemma 5.5 there are only finitely many equivalence classes of maximal graded shortening quotients of a graded limit group.

So far we have modified the construction of maximal shortening quotients presented in section 5 to the graded setup. However, since in general the graded modular group of a graded limit group does not contain the (ungraded) modular group of the graded limit group (as a limit group), graded shortening quotients of a graded limit group need not be proper quotients of it. Furthermore, to get graded shortening quotients we naturally require that the graded abelian JSJ decomposition is 
non-trivial. However, in general the graded abelian JSJ decomposition of a graded limit group may be trivial. To view some of these phenomenas we present the following two examples:

(1) Let $\mathrm{G}(x, p)$ be a graded limit group that is isomorphic to a non-abelian free group, and suppose that the graded abelian JSJ decomposition of $\mathrm{G}(x, p)$ is: $\mathrm{G}(x, p)=\mathrm{P} *_{\mathrm{C}} \mathrm{B}$, where $\mathrm{C}$ is cyclic and $\mathrm{P}$ is the parameter subgroup. Since $\mathrm{G}(x, p)$ is isomorphic to a free group, there is clearly an embedding: $h: \mathrm{G}(x, p) \rightarrow \mathrm{F}_{k}$, for which the image of the generator of the cyclic group $\mathrm{C}$ has no non-trivial roots in $\mathrm{F}_{k}$. In this case for every graded modular automorphism $\varphi \in \operatorname{GMod}(\mathrm{G}(x, p)), h \circ \varphi$ is an embedding of $\mathrm{G}(x, p)$ into $\mathrm{F}_{k}$. Hence, $\mathrm{G}(x, p)$ has a shortening quotient that is isomorphic to it.

(2) Let $\mathrm{G}(x, p)$ be a surface group, and suppose that $\mathrm{G}(x, p)=\mathrm{P} *_{\mathrm{C}} \mathrm{B}$, where $\mathrm{C}$ is cyclic and $\mathrm{P}$ is the parameter subgroup. Then it is possible to find a sequence of homomorphisms $h_{n}: \mathrm{G}(x, p) \rightarrow \mathrm{F}_{k}$, for which the corresponding sequence of graded shortest homomorphisms $h_{n} \circ \varphi_{n}$ converge to a group that is isomorphic to $\mathrm{G}(x, p)$.

As we have already indicated, unlike (ungraded) limit groups, to understand the variation of the set of restricted homomorphisms from a graded limit group to $\mathrm{F}_{k}$ with a change of the defining parameters, we will need to study the structure of graded limit groups with a trivial principal graded abelian JSJ decomposition.

Definition 10.2. — Let $\operatorname{Glim}(x, p, a)$ be a graded limit group that does not split into a free product in which AP can be conjugated into one of the factors. If $\operatorname{Glim}(x, p, a)$ has a trivial graded abelian JSJ decomposition it is called rigid graded limit group. If $\operatorname{Glim}(x, p, a)$ has a non-trivial graded abelian JSJ decomposition, and a (unique) maximal graded shortening quotient $\operatorname{GMSQ}(x, p, a)$ that is isomorphic to $\operatorname{Glim}(x, p, a), \operatorname{Glim}(x, p, a)$ is called solid graded limit group.

To study rigid and solid graded limit groups we need to introduce the notion of flexible graded limit groups.

Definition 10.3. - Let Glim $(x, p, a)$ be either a rigid or a solid graded limit group. A sequence of homomorphisms $\left\{h_{m}: \operatorname{Glim}(x, p, a) \rightarrow \mathrm{F}_{k}\right\}$ is called flexible graded sequence if one of the following holds:

(i) Each homomorphism $h_{m}$ factors as $h_{m}=v_{m} \circ \tau_{m}$, where $\tau_{m}$ : Glim $(x, p, a) \rightarrow \mathrm{F}_{k} *<v>$, $\tau_{m}$ is an epimorphism that maps the subgroup $\mathrm{AP}$ onto the factor $\mathrm{F}_{k}$, and $\mathrm{v}_{m}$ : $\mathrm{F}_{k} *<v>\rightarrow \mathrm{F}_{k}$ restricts to the identity map on $\mathrm{F}_{k}$. Furthermore, $\tau_{m}$ can not be shortened (in $\mathrm{F}_{k} *\langle v\rangle$ ) by an element from the graded modular group $\operatorname{GMod}(\operatorname{Glim}(x, p, a))$.

(ii) Each homomorphism $h_{m}$ does not factor through a free product in which AP is mapped into one of the factors, and can not be shortened by an element from the graded modular 
$\operatorname{group} \mathrm{GMod}(\operatorname{Glim}(x, p, a))$. In addition, for each index $m$ :

$$
\max _{g \in \mathrm{B}_{m}} d_{\mathrm{X}}\left(h_{m}(g), i d .\right)>2^{m} \cdot\left(1+\max _{1 \leqslant j \leqslant u} d_{\mathbf{X}}\left(h_{m}\left(p_{j}\right), i d .\right)\right)
$$

where $\mathbf{B}_{m}$ is the ball of radius $m$ in the Cayley graph of $\operatorname{Glim}(x, p, a)$.

A graded limit group which is the limit of a flexible graded sequence is called a flexible graded quotient of the rigid or solid graded limit $\operatorname{group} \operatorname{Glim}(x, p, a)$.

Lemma 10.4. - Let FlxGlim $(x, p, a)$ be a flexible graded quotient of the rigid or solid graded limit group $\operatorname{Glim}(x, p, a)$. Then:

(i) $\operatorname{Flx} \operatorname{Glim}(x, p, a)$ is not a rigid graded limit group.

(ii) FlxGlim $(x, p, a)$ is a proper quotient of $\operatorname{Glim}(x, p, a)$.

Proof. - If $\operatorname{FlxGlim}(x, p, a)$ splits into a free product in which AP can be conjugated into one of the factors, then $\operatorname{Flx} \operatorname{Glim}(x, p, a)$ is not rigid nor solid, and it can not be isomorphic to $\operatorname{Glim}(x, p, a)$. Hence, we may assume that $\operatorname{Flx} \operatorname{Glim}(x, p, a)$ does not split into a free product in which AP can be conjugated into one of the factors.

By the way a flexible graded sequence of homomorphisms of $\operatorname{Flx} \operatorname{Glim}(x, p, a)$ is defined, it has a subsequence that converges into a stable action of $\operatorname{FlxGlim}(x, p, a)$ on a real tree $\mathrm{Y}$ (see section 1), so that the subgroup AP fixes a point in Y.

By (([Se3], 3.1), see theorem 1.5) FlxGlim $(x, p, a)$ admits a (non-trivial) graph of groups $\Lambda$ from its action on the real tree Y. By that same theorem if the action of Flx $\operatorname{Glim}(x, p, a)$ on Y contains an IET component, then $\operatorname{Flx} \operatorname{Glim}(x, p, a)$ admits a graded abelian splitting. If the action contains an axial component, $\operatorname{Flx} \operatorname{Glim}(x, p, a)$ inherits a graded abelian splitting and if the stabilizer of some (non-degenerate) segment in $\mathrm{Y}$ is non-trivial, it is maximal abelian by lemma 1.3, so $\operatorname{Fl} \operatorname{Glim}(x, p, a)$ splits over a maximal abelian subgroup, hence, $\operatorname{Flx} \operatorname{Glim}(x, p, a)$ admits a graded abelian splitting.

Therefore, in all cases $\operatorname{Flx} \operatorname{Glim}(x, p, a)$ admits a (non-trivial) graded abelian splitting, so the graded abelian JSJ decomposition of $\operatorname{Fl} \operatorname{Glim}(x, p, a)$ is non-trivial, and we get part (i) of the lemma.

If $\operatorname{Glim}(x, p, a)$ is rigid it has a trivial graded abelian JSJ decomposition. Hence, by part (i) FlxGlim $(x, p, a)$ is a proper quotient of it. If $\operatorname{Glim}(x, p, a)$ is solid and $\operatorname{Flx} \operatorname{Glim}(x, p, a)$ is isomorphic to $\operatorname{Glim}(x, p, a)$, then for large enough $m$, it is possible to shorten the homomorphisms $h_{m}: \operatorname{Gim}(x, p, a) \rightarrow \mathrm{F}_{k}$ or the homomorphism $\tau_{m}: \operatorname{Glim}(x, p, a) \rightarrow \mathrm{F}_{k} *<v>$ by pre-composing with elements of the graded modular group $\operatorname{GMod}(\operatorname{Glim}(x, p, a))$, which contradicts the properties of homomorphisms in a flexible sequence. Hence, a flexible graded quotient is a proper quotient of a graded limit group. 
The following is an example of a flexible quotient of a solid limit group. Let $\mathrm{G}(x, p)=\left\langle x_{1}, x_{2}, p\right| p=x_{1}^{3} x_{2}^{-2}>. \mathrm{G}(x, p)$ is a graded limit group and its graded abelian decomposition is the amalgamated product:

$$
\mathrm{G}(x, p)=<p>*_{<p=x_{1}^{3} x_{2}^{-2}>}<x_{1}, x_{2}>
$$

$\mathrm{G}(x, p)$ is isomorphic to a free group of rank 2 , hence, as in the first example presented above it is a solid limit group.

The solid limit group $\mathrm{G}(x, p)$ admits a flexible quotient. Its flexible quotient is a limit group of the form

$$
\begin{aligned}
& F l x(x, p)=\left\langle x_{1}, x_{2}, p\right| p=x_{1}^{3} x_{2}^{-2},\left[x_{1}, x_{2}\right]=1> \\
& =\left\langle p>*_{<p=x_{1}^{3} x_{2}^{-2}>}\left\langle x_{1}, x_{2}\right|\left[x_{1}, x_{2}\right]=1>.\right.
\end{aligned}
$$

Let $\operatorname{Glim}(x, p, a)$ be a rigid or solid graded limit group. On the set of flexible graded quotients of $\operatorname{Glim}(x, p, a)$ we can naturally define a partial order and an equivalence relation, similar to the ones defined on graded limit groups. By the same argument used to prove lemma 5.4, the set of flexible graded quotients of $\operatorname{Glim}(x, p, a)$ contains maximal elements with respect to the partial order, and by the same argument used to prove lemma 5.5 there are only finitely many equivalence classes of maximal flexible graded quotients of $\operatorname{Gim}(x, p, a)$. In the sequel we will need the following definitions associated with rigid and solid graded limit groups and their associated maximal flexible limit quotients.

Definition 10.5. - Let $\operatorname{Rgd}(x, p, a)$ be a rigid limit group and let:

$$
F l x_{1}(x, p, a), \ldots, F l x_{v}(x, p, a)
$$

be the maximal flexible graded quotients of $i$. A homomorphism $h: \operatorname{Rgd}(x, p, a) \rightarrow \mathrm{F}_{k}$ that does not factor through any of the maximal graded flexible quotients

$$
F l x_{1}(x, p, a), \ldots, F l x_{v}(x, p, a)
$$

of $\operatorname{Rgd}(x, p, a)$ is called a rigid homomorphism (solution, specialization) of the rigid graded limit group $\operatorname{Rgd}(x, p, a)$. A homomorphism that does factor through one of the maximal flexible quotients is called a flexible homomorphism (solution, specialization).

Definition 10.6. — Let Sld $(x, p, a)$ be a solid graded limit group and let

$$
F l x_{1}(x, p, a), \ldots, F l x_{v}(x, p, a)
$$

be the maximal flexible quotients of $i$. A homomorphism $h: \operatorname{Sld}(x, p, a) \rightarrow \mathrm{F}_{k}$ for which $h=h^{\prime} \circ \varphi$ where $h^{\prime}$ factors through one of the maximal graded flexible quotients of Sld $(x, p, a)$, and $\varphi$ is a graded modular automorphism of Sld $(x, p, a)$, is called a flexible homomorphism (solution, specialization) of 
the solid graded limit group $\operatorname{Sld}(x, p, a)$. Note that every homomorphism $h: \operatorname{Sld}(x, p, a) \rightarrow \mathrm{F}_{k}$ that factors through a free product of the form $\mathrm{F}_{k} *\langle v\rangle$ so that $\mathrm{AP}$ is mapped into $\mathrm{F}_{k}$, is flexible. A non-flexible homomorphism $h: \operatorname{Sld}(x, p, a) \rightarrow \mathrm{F}_{k}$ is called a solid homomorphism (solution, specialization) of the solid graded limit group $\operatorname{Sld}(x, p, a)$.

Flexible quotients of rigid graded limit groups and flexible quotients of solid graded limit groups contain all the "generic infinite families" of specializations of these graded limit groups. Rigid solutions of rigid graded limit groups are the exceptional single solutions, and solid solutions of solid graded limit groups are the exceptional families of solutions.

Proposition 10.7. - Let $\operatorname{Rgd}(x, p, a)$ be a rigid graded limit group, let $\operatorname{Sld}(x, p, a)$ be a solid graded limit group, and let $p_{0}$ be a specialization of the defining parameters $p$. Then:

(i) There are at most finitely many rigid solutions: $h: \operatorname{Rgd}(x, p, a) \rightarrow \mathrm{F}_{k}$ for which $h(p)=p_{0}$.

(ii) Up to pre-composing with a graded modular automorphism of the solid graded limit group $\operatorname{Sld}(x, p, a)$ there are at most finitely many solid homomorphisms: $h: \operatorname{Sld}(x, p, a) \rightarrow \mathrm{F}_{k}$ for which $h(p)=p_{0}$.

Proof. - Suppose that for some specialization $p_{0}$ of the defining parameters $p$ there are infinitely many rigid homomorphisms of $\operatorname{Rg} d(x, p, a)$. From this infinite set of homomorphisms it is possible to extract a flexible subsequence, from which it is possible to extract a convergent flexible subsequence of homomorphisms. Now, this last flexible sequence converges into a flexible graded quotient of $\operatorname{Rg} d(x, p, a)$. Hence, all but at most finite of the rigid homomorphisms of the convergent subsequence factors through a flexible graded quotient of $\operatorname{Rg} d(x, p, a)$, a contradiction to their rigidity, and we get part (i) of the proposition. Part (ii) follows by the same argument starting with an infinite family of solid homomorphisms that are the shortest possible under the action of the graded modular group $\operatorname{GMod}(\operatorname{Sld}(x, p, a))$.

We will call the (ungraded) Makanin-Razborov diagram of a graded limit group obtained using the (ungraded) abelian JSJ decompositions of the various limit groups that appear along the various resolutions, the ungraded Makanin-Razborov diagram. We call each resolution of the ungraded Makanin-Razborov diagram of a graded limit group, an ungraded resolution. The above basic facts concerning graded limit groups and their graded shortening quotients and graded flexible quotients allow us to present the main goal of this section, the (canonical) graded Makanin-Razborov diagram associated with a graded limit group. The graded Makanin-Razborov diagram "encodes" all possible homomorphisms from a graded limit group into the (fixed) free group $\mathrm{F}_{k}$ in a form "compatible" with the parametric description of the set of solutions.

Let $\operatorname{Glim}(x, p, a)$ be a graded limit group. Let $\operatorname{Glim}(x, p, a)=\operatorname{Glim}_{1} * \ldots \operatorname{Glim}_{\ell} * \mathrm{~F}_{g_{1}}$ be a factorization of $\operatorname{Glim}(x, p, a)$, where $\mathrm{AP}<\operatorname{Glim}_{1}, \operatorname{Glim}_{1}$ can not be written as a 
free product in which AP is contained in one of the factors, each of the Glim, ${ }_{i}$ s for $2 \leqslant i \leqslant \ell$ is a freely-indecomposable non-cyclic subgroup of $\operatorname{Glim}(x, p, a)$ and $\mathrm{F}_{g_{1}}$ is a free group. It follows from Grushko's theorem that such a free decomposition of $\operatorname{Glim}(x, p, a)$ is canonical.

Suppose first that Glim $_{1}$ is not rigid or solid. By an analogue of lemma 5.4 the set of graded shortening quotients of the graded limit group Glim 1 contains maximal elements (with respect to the partial order defined above) and by an analogue of lemma 5.5 in the graded case, there are only finitely many equivalence classes of maximal

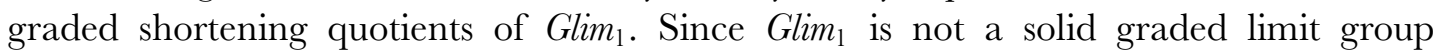
each maximal graded shortening quotient of it is a proper quotient. Let $\mathrm{GM}_{1}, \ldots, \mathrm{GM}_{s}$ be a collection of representatives of equivalence classes of maximal graded shortening quotients of Glim $_{1}$, and let $\eta_{j}:$ Glim $_{1} \rightarrow \mathrm{GM}_{j}$ be the canonical projection maps.

Now, suppose Glim is $_{1}$ rigid or solid. In this case, Glim 1 has finitely many equivalence classes of maximal flexible graded quotients. Let $F l x G l i m_{1}, \ldots, F_{x \rightarrow l i m}$ be a collection of representatives of equivalence classes of maximal flexible graded quotients of Glim $_{1}$, and let $v_{j}:$ Glim $_{1} \rightarrow$ FlxGlim be the canonical projection maps.

We define the graded Makanin-Razborov diagram of the graded limit group $\operatorname{Glim}(x, p, a)$ iteratively. We start by factoring $\operatorname{Glim}(x, p, a)$ as above. From each of the factors Glim $_{2}, \ldots$, Glim $_{\ell}$ we continue by adjoining its (ungraded) Makanin-Razborov diagram. If Glim $_{1}$ is not rigid we continue along $s$ edges going from Glim to $_{1}$ each of its maximal graded shortening quotients $\mathrm{GM}_{1}, \ldots, \mathrm{GM}_{s}$. To each such directed edge we associate the canonical projection $\eta_{j}$.

If Glim $_{1}$ is rigid or solid we continue along $v$ edges going from Glim ${ }_{1}$ to each of its maximal flexible graded quotients $F_{x} G_{l i m}, \ldots$, Flx Glim $v$. To each such directed edge we associate the canonical projection $v_{j}$.

We proceed iteratively. Since a graded shortening quotient of a non-solid graded limit group, and a flexible shortening quotient of a solid or rigid graded limit groups are proper quotients of the graded limit group, the construction terminates by proposition 5.1. Each terminal vertex is either a free group (as in the Makanin-Razborov diagram presented in section 5), or it is a rigid graded limit group $\operatorname{Rgd} \operatorname{Glim}(x, p, a)$ for which for every value of the parameters $p_{0}$ there are at most finitely many possible homomorphisms $h: \operatorname{RgdGlim}(x, p, a) \rightarrow \mathrm{F}_{k}$ that satisfy $h(p)=p_{0}$, or a solid graded limit group $\operatorname{Sld}(x, p, a)$ with no flexible quotients. By construction, up to the equivalence relation of graded and restricted shortening quotients, and flexible graded quotients, the graded Makanin-Razborov diagram of a graded limit group is canonical.

As in the case of (ungraded) limit groups (definition 5.8), we can use the graded Makanin-Razborov diagram to define graded Makanin-Razborov resolutions of a graded limit group. The main purpose of defining graded Makanin-Razborov diagrams and resolutions is the fact these "encode" all the (ungraded) restricted resolutions for every specialization of the defining parameters of the graded limit group. A better 
understanding of this "encoding" requires a better understanding of rigid and solid limit groups and will be obtained in the following sections of this paper and the following papers in the sequel.

As we pointed out in section 8, the study of sets of solutions to equations with coefficients is naturally equivalent to the study of all restricted homomorphisms from a f.g. restricted residually free group into the free group generated by the coefficients. For studying this collection of homomorphisms we have an analogue of theorems 7.2 and 8.4 in the graded case. The proof is a direct modification.

Theorem 10.8. - Let $\mathrm{RF}(x, p, a)$ be a f.g. (graded) residually free group. Then there exists a (canonical) finite collection of graded limit group quotients of $\operatorname{RF}(x, p, a)$ : $\operatorname{Glim}_{1}(x, p, a), \ldots, \operatorname{Glim}_{\ell}(x, p, a)$, and for each $i$ an epimorphism $\tau_{i}: \operatorname{RF}(x, p, a) \rightarrow \operatorname{Glim}_{i}(x, p, a)$ with the following properties:

(i) For each restricted homomorphism $h: \mathrm{RF}(x, p, a) \rightarrow \mathrm{F}_{k}$ there exists some index $i$ (not necessarily unique!) and a restricted homomorphism $f: \operatorname{Glim}_{i}(x, p, a) \rightarrow \mathrm{F}_{k}$ so that $h$ splits as: $h=f \circ \tau_{i}$.

(ii) If $\operatorname{Glim}(x, p, a)$ is a graded quotient of $\mathrm{RF}(x, p, a)$, and $\tau$ is an epimorphism $\tau: \operatorname{RF}(x, p, a) \rightarrow \operatorname{Glim}(x, p, a)$, then there exists some index $i$ and an epimorphism $\lambda: \operatorname{Glim}_{i}(x, p, a) \rightarrow \operatorname{Glim}(x, p, a)$ so that $\tau=\lambda \circ \tau_{i}$.

(iii) The couples $\left\{\left(\operatorname{Glim}_{i}(x, p, a), \tau_{i}\right)\right\}$ represent distinct equivalence classes, and if $i \neq j$ then the couple $\left(\operatorname{Glim}_{i}(x, p, a), \tau_{i}\right)$ can not be compared with $\left(\operatorname{Glim}_{j}(x, p, a), \tau_{j}\right)$ in the partial order defined on these couples.

(iv) If $\operatorname{Glim}_{1}(x, p, a), \ldots, \hat{\operatorname{Glm}}_{u}(x, p, a)$ and $\left\{\widehat{\tau}_{j}: \operatorname{RF}(x, p, a) \rightarrow \hat{G l i m}_{j}(x, p, a)\right\}$ is another collection of graded quotients of $\mathrm{RF}(x, p, a)$ and epimorphisms from $\mathrm{RF}(x, p, a)$ to the graded limit groups $\operatorname{Glim}_{i}(x, p, a)$ 's that satisfy properties (i)-(iii) then $u=\ell$ and up to permutation, the couple $\left(\operatorname{Glim}_{i}(x, p, a), \tau_{i}\right)$ is equivalent to the couple $\left(\operatorname{Glim}_{i}(x, p, a), \widehat{\tau}_{i}\right)$.

We have already modified the construction of the Makanin-Razborov diagram of a limit group to the graded case. Having such diagram for a graded limit group, theorem 10.9 allows us to modify the construction of the Makanin-Razborov diagram associated with a residually free group presented in section 8 to the graded case.

\section{The Singular Locus}

The main purpose for modifying limit groups and their Makanin-Razborov diagrams and resolutions to the graded category is the ability to use the graded objects in order to understand the variation of sets of solutions with a change in the values of the defining parameters. Such analysis is crucial in studying the structure of elementary sets and elementary predicates as we will see later in the sequel. 
Since the collection of graded resolutions of a graded limit group $\operatorname{Glim}(x, p, a)$ "encodes" all the restricted homomorphisms of the group $\operatorname{Glim}\left(x, p_{0}, a\right)$ for any possible specialization $p_{0}$ of the set of defining parameters $p$, to understand the variation in the set of solutions with a change of the defining parameters we need to look closely at all the possible degenerations in the graded resolutions of $\operatorname{Glim}(x, p, a)$ for all possible values of the defining parameters. Our aim in this section is to associate finitely many singular graded limit groups with each rigid and solid limit group in a graded resolution of the graded Makanin-Razborov diagram. The finite collection of singular limit groups associated with a rigid (or solid) limit group in a graded resolution describe all the possible degenerations in the part of the graded resolution that terminates at the rigid (or solid) graded limit group.

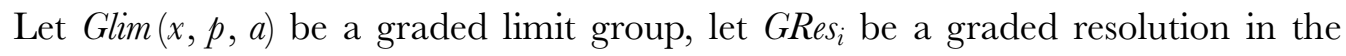
graded Makanin-Razborov diagram of $\operatorname{Gim}(x, p, a)$ and let $\operatorname{Rgd} \operatorname{Glim}(y, p, a)$ be a rigid limit group in this resolution.

Definition 11.1. - We say that the specialization $\left(y_{0}, p_{0}, a\right)$ of the rigid limit group $\operatorname{RgdGlim}(y, p, a)$ belongs to the singular locus of the tuple $\left(\operatorname{RgdGlim}(y, p, a), \operatorname{GRes}_{i}\right)$ if for all homomorphisms $h: \operatorname{Glim}(x, p, a) \rightarrow \mathrm{F}_{k}$ that factor through the resolution GRes and $_{i}$ the specialization $\left(y_{0}, p_{0}, a\right)$ at least one of the graded abelian JSJ decompositions of the graded limit groups along the graded resolution GRes remains degenerate, i.e., at least one of the following possible degenerations in the graded abelian JSJ decompositions occurs:

(i) An edge group is always mapped to the trivial group in $\mathrm{F}_{k}$.

(ii) A non-abelian vertex group is always mapped to a cyclic subgroup in $\mathrm{F}_{k}$.

(iii) $A \mathrm{QH}$ vertex group is always mapped to a cyclic subgroup in $\mathrm{F}_{k}$.

(Note that (iii) can be viewed as a special case of (ii), but we prefer to list it as an additional separate case.)

Clearly, there are only finitely many ways in which one of the graded abelian JSJ decompositions of the graded limit groups along the graded resolution GRes can $_{i}$ get degenerate, so the singular locus of $\left(\operatorname{Rgd} \operatorname{Glim}(x, p, a), \operatorname{GRes}_{i}\right)$ can be stratified according to the degenerate parts of these graded abelian JSJ decompositions.

Theorem 11.2. - There exist finitely many systems of equations $\Sigma_{1}(y, p, a), \ldots, \Sigma_{q}(y, p, a)$ so that a specialization $\left(y_{0}, p_{0}, a\right)$ of the rigid limit group $\operatorname{RgdGlim}(y, p, a)$ belongs to the singular locus of the tuple $\left(\operatorname{RgdGlim}(y, p, a)\right.$, GRes $\left._{i}\right)$ if and only if it satisfies one of the systems $\Sigma_{1}, \ldots, \Sigma_{q}$.

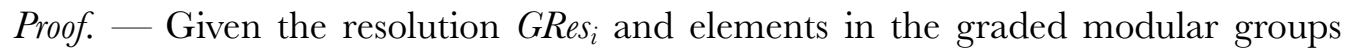
of each of the graded limit groups in the resolution GRes, a degeneration in one of the graded abelian JSJ decomposition of one of the graded limit groups can be expressed as an equation in the variables $(y, p)$. Hence, a specialization $\left(y_{0}, p_{0}, a\right)$ of 
$\operatorname{RgdGlim}(y, p, a)$ belongs to the singular locus of the tuple $\left(\operatorname{RgdGlim}(y, p, a), \operatorname{GRes}_{i}\right)$ if $\left(y_{0}, p_{0}, a\right)$ satisfies an infinite system of equations parameterized by the product of the graded modular groups of the different graded limit groups in the graded resolution GRes. $_{i}$. Since by Guba's theorem [Gu] every infinite system of equations in a free group is equivalent to a finite one, the theorem follows.

Definition 11.3. - We say that a graded limit $\operatorname{group} \operatorname{SngGlim}(y, p, a)$ is a singular graded limit group of the tuple $\left(\operatorname{RgdGlim}(y, p, a)\right.$, GRes $\left._{i}\right)$ if it is a maximal graded limit group corresponding to one of the systems of equations $\Sigma_{1}, \ldots, \Sigma_{q}$ constructed in theorem 11.2. Clearly, this associates canonically finitely many singular limit groups with every tuple $\left(\operatorname{RgdGlim}(x, p, a), \operatorname{GRes}_{i}\right)$.

In a similar way we associate (canonically) finitely many singular limit groups with every tuple of a graded resolution and a solid graded limit group in it. Let $\operatorname{Glim}(x, p, a)$ be a graded limit group, let $\operatorname{RRes}_{i}$ be a graded resolution in the graded Makanin-Razborov diagram of $\operatorname{Glim}(x, p, a)$ and let $\operatorname{SldGlim}(y, p, a)$ be a rigid limit group in this resolution.

Definition 11.4. - We say that the specialization $\left(y_{0}, p_{0}, a\right)$ of the solid limit group $\operatorname{SldGlim}(y, p, a)$ belongs to the singular locus of the tuple $\left(\operatorname{SldGlim}(y, p, a), \operatorname{GRes}_{i}\right)$ if for all homomorphisms $h: \operatorname{Glim}(x, p, a) \rightarrow \mathrm{F}_{k}$ that factor through the resolution GRes and $_{i}$ the specialization $\left(y_{0}, p_{0}, a\right)$, at least one of the abelian graded JSJ decompositions of the graded limit groups along the graded resolution GResi remains degenerate (note that by all homomorphisms that factor through graded resolution GRes and the specialization $\left(y_{0}, p_{0}, a\right)$ we allow a possible pre-composition with a graded modular automorphism of the solid graded limit $\operatorname{group} \operatorname{SldGlim}(y, p, a)$ itself).

Like in the rigid case there exist finitely many systems of equations $\Sigma_{1}(y, p, a), \ldots, \Sigma_{q}(y, p, a)$ so that a specialization $\left(y_{0}, p_{0}, a\right)$ belongs to the singular locus of the tuple (SldGlim $\left.(y, p, a), \operatorname{GRes}_{i}\right)$ if and only if it satisfies one of the systems $\Sigma_{1}, \ldots, \Sigma_{q}$.

We say that a graded limit group $\operatorname{SngGlim}(y, p, a)$ is a singular graded limit group of the tuple $\operatorname{SldGlim}(y, p, a)$, GRes $\left._{i}\right)$ if it is a maximal graded limit group corresponding to one of the systems of equations $\Sigma_{1}, \ldots, \Sigma_{q}$. Clearly, this associates canonically finitely many singular limit groups with every tuple (SldGlim $(x, p, a)$, GRes $\left._{i}\right)$.

\section{Multi-graded Makanin-Razborov Diagrams}

In the previous sections 9-11 we have analyzed the structure of parameterized sets of solutions to systems of equations in a free group, where the sets of solutions were parameterized by some subgroup of parameters $\mathrm{P}=\left\langle p_{1}, \ldots, p_{u}\right\rangle$. The goal of this section is a generalization of the objects introduced in the previous sections, to study sets of solutions to systems of equations parameterized by one subgroup, to the study of sets of solutions to systems of equations parameterized by finitely many subgroups. As we will see in the sequel such parameterization appears in the analysis 
of projections of varieties (Diophantine sets), that is crucial in the analysis of sentences and elementary sets defined over a free group.

With a finite system of equations with parameters $\Phi$ over a free group $\mathrm{F}_{k}=\left\langle a_{1}, \ldots, a_{k}\right\rangle$ it is natural to associate a f.p. group $\mathrm{G}(\Phi)$. If the system $\Phi$ is defined by the coefficients $a_{1}, \ldots, a_{k}$, the parameters $p_{1}, \ldots, p_{u}$ and $r_{1}^{1}, \ldots, r_{m_{1}}^{1}, \ldots, r_{1}^{\ell}, \ldots, r_{m_{\ell}}^{\ell}$, the unknowns $x_{1}, \ldots, x_{n}$ and the equations:

$$
\begin{gathered}
w_{1}\left(a_{1}, \ldots, a_{k}, p_{1}, \ldots, p_{u}, r_{1}^{1}, \ldots, r_{m_{\ell}}^{\ell}, x_{1}, \ldots, x_{n}\right)=1 \\
\vdots \\
w_{s}\left(a_{1}, \ldots, a_{k}, \dot{p}_{1}, \ldots, p_{u}, r_{1}^{1}, \ldots, r_{m_{\ell}}^{\ell}, x_{1}, \ldots, x_{n}\right)=1
\end{gathered}
$$

we set the associated f.p. group $\mathrm{G}(\Phi)$ to be:

$$
\mathrm{G}(\Phi)=<a_{1}, \ldots, a_{k}, p_{1}, \ldots, p_{u}, r_{1}^{1}, \ldots, r_{m_{\ell}}^{\ell}, x_{1}, \ldots, x_{n} \mid w_{1}, \ldots, w_{s}>
$$

Clearly, every solution of the system $\Phi$ corresponds to a homomorphism $h: \mathrm{G}(\Phi) \rightarrow \mathrm{F}_{k}$ for which $h\left(a_{i}\right)=a_{i}$, and every such homomorphism corresponds to a solution of the system $\Phi$.

In sections 9-11 we studied the variation of the structure of the set of solutions with a change of the defining parameters. In this section we study the variation of the set of solutions with a change of the specialization of defining parameters $\mathrm{P}=\left\langle p_{1}, \ldots, p_{u}\right\rangle$, and the conjugacy classes of the specializations of the subgroups $\mathrm{R}_{1}=\left\langle r_{1}^{1}, \ldots, r_{m_{1}}^{1}>, \ldots, \mathbf{R}_{\ell}=<r_{1}^{\ell}, \ldots, r_{m_{\ell}}^{\ell}\right.$. To analyze the variation in the structure of the set of solutions with a change of the specialization of the defining parameters $\mathrm{P}$ and the conjugacy classes of the specializations of the subgroups $\mathrm{R}_{1}, \ldots, \mathrm{R}_{\ell}$ we will need to look at a special class of graded limit groups which we call multi-graded limit groups.

We set $\mathrm{G}=\left\langle x_{1}, \ldots, x_{k}, p_{1}, \ldots, p_{u}, r_{1}^{1}, \ldots, r_{m_{\ell}}^{\ell}, a_{1}, \ldots, a_{k}\right\rangle$ to be a f.g. group. For brevity we will denote this (fixed) generating set by $\mathrm{G}=\left\langle g_{1}, \ldots, g_{m}\right\rangle$, and $\mathrm{F}_{k}$ to be a free group with a fixed basis $\mathrm{F}_{k}=\left\langle a_{1}, \ldots, a_{k}\right\rangle$. With this notation our goal is to get a structure theory for understanding the set of all homomorphisms:

$$
\operatorname{Hom}\left(\left(\mathrm{G},\left\{p_{j}\right\},\left\{r_{q}\right\},\left\{a_{i}\right\}\right),\left(\mathrm{F}_{k},\left\{a_{i}\right\}\right)\right)=\left\{h \mid h: \mathrm{G} \rightarrow \mathrm{F}_{k}, h\left(a_{i}\right)=a_{i}\right\}
$$

with a change in the specializations of the parameters $\left\{p_{j}\right\}$ and the conjugacy classes of the specializations of the subgroups $\mathrm{R}_{1}, \ldots, \mathrm{R}_{\ell}$. We set $\mathrm{X}$ to be the Cayley graph of $\mathrm{F}_{k}$ with respect to its given basis $a_{1}, \ldots, a_{k}$. Given any homomorphism $h \in \operatorname{Hom}\left(\mathrm{G}, \mathrm{F}_{k}\right)$, $\mathrm{G}$ admits a natural action $\lambda_{h}$ on $\mathrm{X}$ given by $\lambda_{h}(g, x)=h(g)(x)$ for every $g \in \mathrm{G}$ and $x \in \mathrm{X}$.

Definition 12.1. - Let $\mathrm{G}$ be a f.g. group and let $h_{n}: \mathrm{G}(x, r, p, a) \rightarrow \mathrm{F}_{k}$ be a sequence of homomorphisms. If $\left\{\lambda_{h_{n}}\right\}$, a sequence of actions of $\mathrm{G}$ on $\mathrm{X}$, the Cayley graph of $\mathrm{F}_{k}$, that corresponds 
to the sequence of homomorphisms $\left\{h_{n}\right\}$, converges in the Gromov-Haussdorff topology, we say that the sequence $\left\{h_{n}\right\}$ converges. We call the obtained limit group, a multi-graded limit group.

A multi-graded limit group is in particular a limit group, so every f.g. multigraded limit group is f.p. and every f.g. graded limit group is $\omega$-residually free by theorem 4.6. To construct the (canonical) multi-graded Makanin-Razborov diagram of a multi-graded limit group, which is the fundamental tool in our analysis of the variation of sets of solutions to systems of equations with a change in the specialization of the parameters $\mathrm{P}$ and the conjugacy class of the specializations of the subgroups $\mathrm{R}_{1}, \ldots, \mathbf{R}_{m}$, we need to study the algebraic structure of multi-graded limit groups "relative" to their multi-grading. To do that we need to construct the multi-graded abelian JSJ decomposition of a multi-graded limit group. These constructions are all generalizations of their graded analogues presented in sections 9-11.

For the rest of this section let $\mathrm{G}(x, r, p, a)$ be a f.g. group, let $\left\{h_{m}\right.$ : $\left.\mathrm{G}(x, r, p, a) \rightarrow \mathrm{F}_{k}\right\}$ be a convergent multi-graded sequence of homomorphisms, and let $\operatorname{MGlim}(x, r, p, a)$ be the corresponding graded limit group. We set $\eta: \mathrm{G}(x, r, p, a) \rightarrow$ $\operatorname{MGlim}(x, r, p, a)$ to be the canonical epimorphism and $\mathrm{AP}<\operatorname{MGlim}(x, r, p, a)$ to be the subgroup: $\mathrm{AP}=\left\langle\eta\left(p_{1}\right), \ldots, \eta\left(p_{u}\right), \eta\left(a_{1}\right), \ldots, \eta\left(a_{k}\right)>\right.$. The abelian JSJ decomposition of a freely-indecomposable limit group "encodes" all the possible splittings of such limit group with abelian edge groups. In studying multi-graded limit groups we will be interested only in abelian splittings in which the subgroup AP and the subgroups $\mathbf{R}_{1}, \ldots, \mathbf{R}_{m}$ are elliptic. The construction of the abelian JSJ decomposition of a limit group described in section 2 naturally generalizes to a multi-graded abelian JSJ decomposition of a multi-graded limit group.

Theorem 12.2 (cf. 9.2). - Suppose MGlim $(x, r, p, a)=L_{0} * \ldots * \mathrm{~L}_{v}$ is the most refined free decomposition in which $\mathrm{AP}<\mathrm{L}_{0}$ and each of the subgroups $\mathrm{R}_{j}$ can be conjugated into one of the factors $\mathrm{L}_{i}$. With each of the factors we associate a multi-graded abelian JSJ decomposition. If none of the subgroups $\mathrm{R}_{j}$ can be conjugated into $\mathrm{L}_{i}$ we associate with it its abelian JSJ decomposition. If $\mathrm{L}_{i}$ is $\mathrm{L}_{0}$ or it contains a conjugate of one of the subgroups $\mathrm{R}_{j}$ then there exists a (relatively) reduced unfolded (perhaps trivial) splitting of the factor $\mathrm{L}_{i}$ with abelian edge groups, which we call a multi-graded abelian JSJ (Jaco-Shalen-Fohannson) decomposition of the factor $\mathrm{L}_{i}$ with the following properties:

(i) AP is elliptic in the multi-graded abelian JSJ decomposition of $\mathrm{L}_{0}$. If a subgroup $\mathrm{R}_{j}$ can be conjugated into the factor $\mathrm{L}_{i}$, then $\mathrm{R}_{j}$ is elliptic in the multi-graded abelian JSJ decomposition of $\mathrm{L}_{i}$.

(ii) Every (multi-graded) canonical maximal $\mathrm{QH}$ subgroup (CMQ) of $\mathrm{L}_{i}$ is conjugate to a vertex group in the multi-graded JSJ decomposition. Every (multi-graded) $\mathrm{QH}$ subgroup of $\operatorname{MGlim}(x, r, p, a)$ can be conjugated into one of the CMQ subgroups of $\mathrm{L}_{i}$. Every vertex group in the multi-graded JSJ decomposition which is not a CMQ subgroup of $\mathrm{L}_{i}$ 
is elliptic in any abelian splitting of $\mathrm{L}_{i}$ under consideration (i.e., in one in which $\mathrm{AP}$ and the $\mathrm{R}_{j}$ 's are elliptic).

(iii) A one edge (multi-graded) abelian splitting $\operatorname{MGlim}(x, r, p, a)=\mathrm{D} *_{\mathrm{A}} \mathrm{E}$ or $\mathrm{L}_{i}=\mathrm{D} *_{\mathrm{A}}$ in which $\mathrm{AP}$ and the $\mathrm{R}_{j}$ 's are elliptic which is hyperbolic in another such elementary abelian splitting is obtained from the multi-graded abelian JSJ decomposition of $\mathrm{L}_{i}$ by cutting a 2-orbifold corresponding to a (multi-graded) $\mathrm{CMQ}$ subgroup of $\mathrm{L}_{i}$ along a weakly essential s.c.c.

(iv) Let $\Theta$ be a one edge (multi-graded) splitting along an abelian subgroup $\mathrm{L}_{i}=\mathrm{D} *_{\mathrm{A}} \mathrm{E}$ or $\mathrm{L}_{i}=\mathrm{D} *_{\mathrm{A}}$ in which $\mathrm{AP}$ and the $\mathrm{R}_{j}$ 's are elliptic, which is elliptic with respect to any other one edge (multi-graded) abelian splitting of $\mathrm{L}_{i}$ in which $\mathrm{AP}$ and the $\mathrm{R}_{j}$ 's are elliptic. Then $\Theta$ is obtained from the multi-graded abelian JSJ decomposition of $\mathrm{L}_{i}$ by a sequence of collapsings, foldings and conjugations.

(v) If $\mathrm{JSJ}_{1}$ is another multi-graded abelian JSJ decomposition of $\mathrm{L}_{i}$, then $\mathrm{JSJ}_{1}$ is obtained from the multi-graded abelian JSJ decomposition by a sequence of slidings, conjugations and modifying boundary monomorphisms by conjugations (see section 1 of [Ri-Se2] for these notions).

In section 5 we have used the canonical cyclic JSJ decomposition of a limit group to construct its canonical Makanin-Razborov diagram. In section 10 we modified this construction to obtain the graded Makanin-Razborov diagram for analyzing the variation of the restricted Makanin-Razborov diagram with a change of the defining parameters. To understand the variation of the (ungraded) Makanin-Razborov diagram with a change of the specialization of the parameter subgroup $\mathrm{P}$, and the conjugacy classes of the specializations of the subgroups $\mathrm{R}_{j}$, we need to further modify the construction of the graded Makanin-Razborov diagram to obtain the multi-graded Makanin-Razborov diagram of a multi-graded limit group.

Definition 12.3. - Let $\operatorname{MGlim}(x, r, p, a)$ be a multi-graded limit group and let $\operatorname{MGlim}(x, r, p, a)=\mathrm{L}_{0} * \ldots * \mathrm{~L}_{v}$ be the most refined free decomposition in which $\mathrm{AP}<\mathrm{L}_{0}$ and the subgroups $\mathrm{R}_{j}$ can be conjugated into the different factors $\mathrm{L}_{i}$. Let $\mathrm{L}_{i}$ be one of those factors and suppose that the multi-graded abelian JSJ decomposition of $\mathrm{L}_{i}$ is non-trivial. We define the multigraded modular group $\operatorname{MGMod}\left(\mathrm{L}_{i}\right)$ to be the subgroup of $\operatorname{Aut}\left(\mathrm{L}_{i}\right)$ generated by the following families of automorphisms of the factor $\mathrm{L}_{i}$ :

(i) Dehn twists along edges of the multi-graded abelian JSJ decomposition of $\mathrm{L}_{i}$. The Dehn twists are assumed to fix (elementwise) the vertex stabilized by $\mathrm{AP}$ in case $\mathrm{L}_{i}$ is the factor $\mathrm{L}_{0}$.

(ii) Dehn twists along essential s.c.c. in (multi-graded) CMQ (canonical maximal quadratically hanging) vertex groups in the multi-graded abelian JSJ decomposition of $\mathrm{L}_{i}$. Again, these Dehn twists are assume to fix (elementwise) the vertex stabilized by $\mathrm{AP}$ in case $\mathrm{L}_{i}$ is the factor $\mathrm{L}_{0}$. 
(iii) Let $\mathrm{A}$ be an abelian vertex group in the multi-graded abelian JSJ decomposition of $\mathrm{L}_{i}$. Let $\mathrm{A}_{1}<\mathrm{A}$ be the subgroup generated by all edge groups connected to the vertex stabilized by $\mathrm{A}$ in the multi-graded abelian JSJ decomposition of $\mathrm{L}_{i}$. Every automorphism of $\mathrm{A}$ that fixes $\mathrm{A}_{1}$ (elementwise) can be naturally extended to an automorphism of the ambient factor $\mathrm{L}_{i}$ that fixes the vertex stabilized by $\mathrm{AP}$ and conjugates of the other subgroups $\mathrm{R}_{j}$. We call these generalized Dehn twists and they form the third family of automorphisms that generate $\operatorname{MGMod}\left(\mathrm{L}_{i}\right)$.

We say that a homomorphism $h: \operatorname{MGlim}(x, r, p, a) \rightarrow \mathrm{F}_{k}$ is the same multi-graded modular class as the homomorphism $\widehat{h}: \operatorname{MGlim}(x, r, p, a) \rightarrow \mathrm{F}_{k}$ if $\widehat{h}=h \circ \varphi$, and $\varphi$ is a multi-graded modular automorphism of $\operatorname{MGlim}(x, r, p, a)$, i.e., $\varphi \in \operatorname{MGlim}(x, r, p, a)$.

Let $\mathrm{L}_{i}$ be a factor in the most refined free decomposition of the multi-graded limit group $\operatorname{MGlim}(x, r, p, a)$ in which the subgroups $\mathrm{AP}$ and $\mathrm{R}_{j}$ 's can be conjugated into the various factors. Once we fix a generating set for the factor $\mathrm{L}_{i}$, given a homomorphism $h: \mathrm{L}_{i} \rightarrow \mathrm{F}_{k}$ we can choose the shortest homomorphisms in the multigraded modular class of $h$. A multi-graded limit group obtained from a convergent sequence of homomorphisms $h: \mathrm{L}_{i} \rightarrow \mathrm{F}_{k}$ which are the shortest in their multi-graded modular class, is called a multi-graded shortening quotient of the factor $\mathrm{L}_{i}$.

On the set of multi-graded shortening quotients of the factor $\mathrm{L}_{i}$ we can naturally define a partial order and an equivalence relation, similar to the ones defined in section 5 for shortening quotients of limit groups. By the same argument used to prove lemma 5.4 the set of multi-graded shortening quotients of $\mathrm{L}_{i}$ contains maximal elements with respect to the partial order, and by the same argument used to prove lemma 5.5 there are only finitely many equivalence classes of maximal multi-graded shortening quotients of the factor $\mathrm{L}_{i}$.

So far we have modified the construction of maximal shortening quotients presented in section 5 to the multi-graded setup. However, multi-graded shortening quotients of a multi-graded limit group need not be proper quotients of it. Furthermore, to get multi-graded shortening quotients we naturally require that the multi-graded abelian JSJ decomposition of the factor $\mathrm{L}_{i}$ is non-trivial. Unlike (ungraded) limit groups, to understand the variation of the set of restricted homomorphisms from a graded limit group to $\mathrm{F}_{k}$ with a change of the specialization of the defining parameters $\mathrm{P}$ and the conjugacy classes of the specializations of the subgroups $\mathrm{R}_{j}$, we will need to study the structure of multi-graded limit groups with a trivial principal graded abelian JSJ decomposition.

Definition 12.4. - Let $\mathrm{L}_{i}$ be a factor in the most refined free decomposition of a multi-graded limit group MGlim $(x, r, p, a)$ in which the subgroups $\mathrm{AP}$ and $\mathrm{R}_{1}, \ldots, \mathrm{R}_{m}$ can be conjugated into the various factors. If the factor $\mathrm{L}_{i}$ has a trivial multi-graded abelian JSJ decomposition it is called rigid multi-graded limit group. If $\mathrm{L}_{i}$ has a non-trivial multi-graded JSJ decomposition, and a (unique) 
maximal multi-graded shortening quotient $\operatorname{GMSQ}(x, r, p, a)$ that is isomorphic to $\mathrm{L}_{i}, \mathrm{~L}_{i}$ is called solid multi-graded limit group.

To study rigid and solid multi-graded limit groups we need to introduce the notion of flexible multi-graded limit groups.

Definition 12.5. - Let $\mathrm{L}_{i}$ be either a rigid or a solid factor of a multi-graded limit group $\operatorname{MGlim}(x, r, p, a) . A$ sequence of homomorphisms $\left\{h_{m}: \mathrm{L}_{i} \rightarrow \mathrm{F}_{k}\right\}$ is called flexible multi-graded sequence if one of the following holds:

(i) Each homomorphism $h_{m}$ factors as $h_{m}=v_{m} \circ \tau_{m}$, where $\tau_{m}: \mathrm{L}_{i} \rightarrow \mathrm{F}_{k} *\left\langle v>\right.$ and $\tau_{m}$ is an epimorphism that maps the subgroup $\mathrm{AP}$ onto the factor $\mathrm{F}_{k}$ and the subgroups $\mathrm{R}_{j}$ into a conjugate of $\mathrm{F}_{k}$ in $\mathrm{F}_{k} *\langle v\rangle$, and $\mathrm{v}_{m}: \mathrm{F}_{k} *\langle v\rangle \rightarrow \mathrm{F}_{k}$ restricts to the identity on $\mathrm{F}_{k}$. Furthermore, $\tau_{m}$ can not be shortened (in $\mathrm{F}_{k} *\langle v\rangle$ ) by an element from the graded modular group $\operatorname{MGMod}\left(\mathrm{L}_{i}\right)$.

(ii) Each homomorphism $h_{m}$ does not factor through a free product as in (i), and can not be shortened by an element from the multi-graded modular group $\operatorname{MGMod}\left(\mathrm{L}_{i}\right)$. In addition, if $\mathrm{L}_{i}=\mathrm{L}_{0}$ then for each index $m$ :

$$
\begin{aligned}
& \max _{g \in \mathrm{B}_{m}} d_{\mathrm{X}}\left(h_{m}(g), i d .\right)>2^{m} \cdot\left(1+\max _{1 \leqslant j \leqslant u} d_{\mathrm{X}}\left(h_{m}\left(p_{j}\right), i d .\right)+\right. \\
& \max _{1 \leqslant j \leqslant \ell} \min _{f \in \mathrm{F}_{k}}\left(d_{\mathrm{X}}\left(f h_{m}\left(r_{1}^{j}\right) f^{-1}, i d .\right)+\ldots+\left(d_{\mathrm{X}}\left(f h_{m}\left(r_{m_{j}}^{j}\right) f^{-1}, i d .\right)\right)\right.
\end{aligned}
$$

where $\mathrm{B}_{m}$ is the ball of radius $m$ in the Cayley graph of $\mathrm{L}_{i}$. If $\mathrm{L}_{i}$ is not the factor $\mathrm{L}_{0}$, i.e., if $\mathrm{AP}$ is not a subgroup of $\mathrm{L}_{i}$, then for each index $m$ :

$$
\begin{aligned}
& \min _{f \in \mathrm{F}_{k}} \max _{g \in \mathrm{B}_{m}} d_{\mathrm{X}}\left(f h_{m}(g) f^{-1}, i d_{.}\right)>2^{m} \cdot\left(1+\max _{1 \leqslant j \leqslant \ell} \min _{f \in \mathrm{F}_{k}}\left(d_{\mathrm{X}}\left(f h_{m}\left(r_{1}^{j}\right) f^{-1}, i d .\right)\right.\right. \\
& +\ldots+\left(d_{\mathrm{X}}\left(f h_{m}\left(r_{m_{j}}^{j}\right) f^{-1}, i d .\right)\right) .
\end{aligned}
$$

A multi-graded limit group which is the limit of a flexible multi-graded sequence is called a flexible multi-graded quotient of the rigid or solid multi-graded limit group $\mathrm{L}_{i}$.

Lemma 12.6. - Let Flx $(x, r, p, a)$ be a flexible multi-graded quotient of the rigid or solid graded limit factor $\mathrm{L}_{i}$. Then:

(i) FlxGlim $(x, p, a)$ is not a rigid multi-graded limit group.

(ii) $\operatorname{Flx} \operatorname{Glim}(x, p, a)$ is a proper quotient of $\mathrm{L}_{i}$.

Proof. - Identical with the proof of lemma 10.5 .

Let $\operatorname{MGlim}(x, r, p, a)$ be a rigid or solid multi-graded limit group. On the set of flexible multi-graded quotients of $\operatorname{MGlim}(x, r, p, a)$ we can naturally define a partial 
order and an equivalence relation, similar to the ones defined on multi-graded limit groups. By the same argument used to prove lemma 5.4, the set of flexible graded quotients of $\operatorname{MGlim}(x, r, p, a)$ contains maximal elements with respect to the partial order, and by the same argument used to prove lemma 5.5 there are only finitely many equivalence classes of maximal flexible multi-graded quotients of $\operatorname{MGlim}(x, r, p, a)$. For the sequel we will need the following definitions associated with rigid and solid multigraded limit groups and their associated maximal flexible limit quotients.

Definition 12.7. — Let $\operatorname{Rgd}(x, r, p, a)$ be a rigid limit group and let

$$
F l x_{1}(x, r, p, a), \ldots, F l x_{v}(x, r, p, a)
$$

be the maximal flexible multi-graded quotients of it. A homomorphism $h: \operatorname{Rgd}(x, r, p, a) \rightarrow \mathrm{F}_{k}$ that does not factor through any of the maximal multi-graded flexible quotients $F l x_{1}(x, r, p, a), \ldots$, $F l x_{v}(x, r, p, a)$ of $\operatorname{Rgd}(x, r, p, a)$ is called a rigid homomorphism (solution) of the rigid multi-graded limit group $\operatorname{Rgd}(x, r, p, a)$. A homomorphism that does factor through one of the maximal flexible quotients is called a flexible homomorphism (solution).

Definition 12.8. - Let Sld $(x, r, p, a)$ be a solid multi-graded limit group and let $F l x_{1}(x, r, p, a), \ldots, F l x_{v}(x, r, p, a)$ be the maximal flexible quotients of it. A homomorphism $h: \operatorname{Sld}(x, r, p, a) \rightarrow \mathrm{F}_{k}$ for which $h=h^{\prime} \circ \varphi$ where $h^{\prime}$ factors through one of the maximal multi-graded flexible quotients of Sld $(x, r, p, a)$, and $\varphi$ is a multi-graded modular automorphism of Sld $(x, r, p, a)$, is called a flexible homomorphism (solution) of the solid multi-graded limit group $\operatorname{Sld}(x, r, p, a)$. Note that every homomorphism of $\operatorname{Sld}(x, r, p, a) \rightarrow \mathrm{F}_{k}$ that factors through a free product of the form $\mathrm{F}_{k} *\langle v\rangle$ so that $\mathrm{AP}$ and the subgroups $R_{j}$ are mapped into $\mathrm{F}_{k}$, is flexible. $A$ non-flexible homomorphism $h: \operatorname{Sld}(x, r, p, a) \rightarrow \mathrm{F}_{k}$ is called a solid homomorphism (solution) of the solid multi-graded limit group $\operatorname{Sld}(x, r, p, a)$.

Flexible quotients of rigid multi-graded limit groups and flexible quotients of solid multi-graded limit groups contain all the "generic infinite families" of specializations of these multi-graded limit groups. Rigid solutions of rigid multi-graded limit groups are the exceptional single solutions, and solid solutions of solid multi-graded limit groups are the exceptional families of solutions.

Proposition 12.9. - Let $\operatorname{Rgd}(x, r, p, a)$ be a rigid multi-graded limit group, let $\operatorname{Sld}(x, r, p, a)$ be a solid multi-graded limit group, and let $p_{0}$ be a specialization of the defining parameters $p$. Then:

(i) There are at most finitely many rigid solutions: $h: \operatorname{Rgd}(x, r, p, a) \rightarrow \mathrm{F}_{k}$ for which $h(p)=p_{0}$ and each of the subgroups $\mathbf{R}_{j}$ are mapped to a conjugate of $h\left(\mathbf{R}_{j}\right)$ (elementwise).

(ii) Up to pre-composing with a graded modular automorphism of the solid graded limit group $\operatorname{Sld}(x, r, p, a)$ there are at most finitely many solid homomorphisms: $h: \operatorname{Sld}(x, r, p, a) \rightarrow$ 
$\mathbf{F}_{k}$ for which $h(p)=p_{0}$ and each of the subgroups $\mathbf{R}_{j}$ are mapped to a conjugate of $h\left(\mathbf{R}_{j}\right)$ (elementwise).

Proof. - Identical with the proof of proposition 10.7 .

The above basic facts concerning multi-graded limit groups and their multigraded shortening quotients and multi-graded flexible quotients allow us to present the main goal of this section, the (canonical) multi-graded Makanin-Razborov diagram associated with a multi-graded limit group.

Let $\operatorname{MGlim}(x, r, p, a)$ be a multi-graded limit group. Let $\operatorname{MGlim}(x, r, p, a)=\mathrm{L}_{0} *$ $\ldots * \mathrm{~L}_{v}$ be the most refined free decomposition of $\operatorname{MGlim}(x, r, p, a)$ so that $\mathrm{AP}<\mathrm{L}_{0}$ and each of the subgroups $\mathrm{R}_{j}$ can be conjugated into one of the $\mathrm{L}_{i}$ 's. Suppose first that a factor $\mathrm{L}_{i}$ contains either the subgroup AP or a conjugate of one of the subgroups $\mathrm{R}_{j}$. Suppose $\mathrm{L}_{i}$ is not rigid or solid. Then by claim 13.2 the set of multi-graded shortening quotients of the factor $\mathrm{L}_{i}$ contains maximal elements (with respect to the partial order defined above) and by an analogue of lemma 5.5 to the multi-graded case there are only finitely many equivalence classes of maximal multi-graded shortening quotients of $\mathrm{L}_{i}$. Since $\mathrm{L}_{i}$ is not a solid multi-graded limit group each maximal multi-graded shortening quotient of it is a proper quotient. Let $\mathrm{MGM}_{1}, \ldots, \mathrm{MGM}_{s}$ be a collection of representatives of equivalence classes of maximal multi-graded shortening quotients of the factor $\mathrm{L}_{i}$, and let $\eta_{q}: \mathrm{L}_{i} \rightarrow \mathrm{MGM}_{q}$ be the canonical projection maps. Note that $\mathrm{MGM}_{q}$ is a multi-graded limit group with respect to some subset of the subgroups $\mathrm{P}$ and $\mathrm{R}_{1}, \ldots, \mathrm{R}_{\ell}$.

Now, suppose $\mathrm{L}_{i}$ is rigid or solid. In this case, $\mathrm{L}_{i}$ has finitely many equivalence classes of maximal flexible multi-graded quotients. Let $F l x_{1}, \ldots, F l x_{t}$ be a collection of representatives of equivalence classes of maximal flexible graded quotients of $\mathrm{L}_{i}$, and let $v_{q}: \mathrm{L}_{i} \rightarrow F l x_{q}$ be the canonical projection maps.

We define the multi-graded Makanin-Razborov diagram of the multi-graded limit group $\operatorname{MGlim}(x, r, p, a)$ iteratively. We start by factoring $\operatorname{MGlim}(x, r, p, a)$ as above. From each of the factors $\mathrm{L}_{i}$ that does not contain the subgroup AP or a conjugate of one of the subgroups $\mathrm{R}_{j}$ we continue by adjoining its (ungraded) Makanin-Razborov diagram presented in section 5. If $\mathrm{L}_{i}$ contains the subgroup AP or a conjugate of one of the $\mathrm{R}_{j}$ 's, and $\mathrm{L}_{i}$ is not rigid or solid we continue along $s$ edges going from $\mathrm{L}_{i}$ to each of its maximal multi-graded shortening quotients $\mathrm{MGM}_{1}, \ldots, \mathrm{MGM}_{s}$. To each such directed edge we associate the canonical projection $\eta_{q}$.

If $\mathrm{L}_{i}$ is rigid or solid we continue along $t$ edges going from $\mathrm{L}_{i}$ to each of its maximal multi-graded flexible quotients $F l x_{1}, \ldots, F l x_{t}$. To each such directed edge we associate the canonical projection $\boldsymbol{v}_{q}$.

We proceed iteratively. Since a multi-graded shortening quotient of a nonsolid multi-graded limit group, and a flexible shortening quotient of a solid or rigid multi-graded limit groups are proper quotients of the multi-graded limit group, the 
construction terminates by proposition 5.1. Each terminal vertex is either a free group (as in the Makanin-Razborov diagram presented in section 5), or it is a rigid multigraded limit group $\operatorname{Rgd}(x, r, p, a)$ for which for every value of the parameters $\mathrm{P}$ and given conjugacy classes of the subgroups $\mathrm{R}_{j}$, there are at most finitely many possible homomorphisms $h: \operatorname{Rgd}(x, r, p, a) \rightarrow \mathrm{F}_{k}$ that obtaining these values and conjugacy classes, or a solid multi-graded limit group $\operatorname{Sld}(x, r, p, a)$ with no flexible quotients. By construction, up to the equivalence relation of multi-graded and restricted shortening quotients, and multi-graded flexible quotients, the multi-graded Makanin-Razborov diagram of a multi-graded limit group is canonical. As in the case of (ungraded) limit groups (definition 5.8), we can use the multi-graded Makanin-Razborov diagram to define multi-graded Makanin-Razborov resolutions of a multi-graded limit group.

\section{REFERENCES}

[Be] M. Bestvina, R-trees in topology, geometry, and group theory, preprint.

[Be-Fel] M. Bestvina and M. Feighn, Stable actions of groups on real trees, Inventiones Math. 121 (1995), 287-321.

[Be-Fe2] M. Bestvina and M. Feighn, Bounding the complexity of simplicial group actions, Inventiones Math. 103 (1991), 449-469.

[De-Po] T. Delzant and L. Potyagallo, Accessibilité hiérarchique, Topology, to appear.

[Du] M. Dunwoody, Groups acting on protrees, 7. London Math. Soc. 56 (1997), 125-136.

[Du-Sa] M. Dunwoody and M. SAgeev, JSJ-splittings for finitely presented groups over slender groups, Inventiones Math. 135 (1999), 25-44.

[Fa] B. FARB, Relatively hyperbolic groups, GAFA 8 (1998), 810-840.

[Fu-Pa] K. Fujinara and P. Papasoglu, JSJ decompositions and complexes of groups, preprint.

[Gu] V. S. Guba, Equivalence of infinite systems of equations in free groups and semigroups to finite subsystems, Math. Zametki 40 (1986), 321-324.

[Kh-My] O. Kharlampovich and A. Myasnikov, Irreducible affine varieties over a free group II, $\mathcal{F}$. of Algebra 200 (1998), 517-570.

[Mal] G. S. Makanin, Equations in a free group, Math. USSR Izvestiya 21 (1983), 449-469.

[Ma2] G. S. Makanin, Decidability of the universal and positive theories of a free group, Math. USSR Izvestiya 25 (1985), 75-88.

[Me] Yu. I. Merzlyakov, Positive formulae on free groups, Algebra i Logika 5 (1966), 257-266.

[Pa] F. Paulin, Outer automorphisms of hyperbolic groups and small actions on R-trees, Arboreal Group Theory (ed. R. C. Alperin), 331-343.

[Ral] A. A. Razborov, On systems of equations in a free group, Math. USSR Izvestiya 25 (1985), 115-162.

[Ra2] A. A. Razborov, On systems of equations in a free group, Ph.D. thesis, Steklov Math. institute (1987).

[Ri-Sel] E. RIPs and Z. SELA, Structure and rigidity in hyperbolic groups I, GAFA 4 (1994), 337-371.

[Ri-Se2] E. RIPS and Z. SeLA, Cyclic splittings of finitely presented groups and the canonical JSJ decomposition, Annals of Mathematics 146 (1997), 53-104.

[Se1] Z. SeLA, The Nielsen-Thurston classification and automorphisms of a free group I, Duke Math. $\mathcal{J}$. 84 (1996), 379-397. 
[Se2] Z. SELA, Structure and rigidity in (Gromov) hyperbolic groups and discrete groups in rank 1 Lie groups II, GAFA 7 (1997), 561-593.

[Se3] Z. SeLA, Acylindrical accessibility for groups, Inventiones Mathematicae 129 (1997), 527-565.

[Se4] Z. SeLA, Endomorphisms of hyperbolic groups I: The Hopf property, Topology 38 (1999), 301-321.

[We] R. Weidmann, The Nielsen method for groups acting on trees, preprint.

Z. S.

Hebrew University

Jerusalem 91904, Israel 